\title{
Faint Tidal Features in Galaxies within the Canada-France-Hawaii Telescope Legacy Survey Wide Fields
}

\author{
Adam M. Atkinson ${ }^{1}$, Roberto G. Abraham ${ }^{1}$ and Annette M. N. Ferguson ${ }^{2}$
}

\begin{abstract}
We present an analysis of the detectability of faint tidal features in galaxies from the widefield component of the Canada-France-Hawaii Telescope Legacy Survey. Our sample consists of 1781 luminous $\left(M_{r^{\prime}}<-19.3 \mathrm{mag}\right)$ galaxies in the magnitude range $15.5<r^{\prime}<17$ mag and in the redshift range $0.04<z<0.2$. Although we have classified tidal features according to their morphology (e.g. streams, shells and tails), we do not attempt to interpret them in terms of their physical origin (e.g. major versus minor merger debris). Instead, we provide a catalog that is intended to provide raw material for future investigations which probe the nature of low surface brightness substructure around galaxies. We find that around $12 \%$ of the galaxies in our sample show clear tidal features at the highest confidence level. This fraction rises to about $18 \%$ if we include systems with convincing albeit weaker tidal features, and to $26 \%$ if we include systems with more marginal features that may or may not be tidal in origin. These proportions are a strong function of rest-frame colour and of stellar mass. Linear features, shells, and fans are much more likely to occur in massive galaxies with stellar masses $>10^{10.5} \mathrm{M}_{\odot}$, and red galaxies are twice as likely to show tidal features than are blue galaxies.
\end{abstract}

Subject headings: galaxies: evolution - galaxies: interactions - catalogs: galaxies - galaxies: formation galaxies: structure

\section{Introduction}

Colliding and interacting galaxies have been the subject of systematic investigations by observers for over 50 years, dating back to the the Catalogue of Interacting Galaxies published by Vorontsov-Velyaminov (1959, 1977), and the Atlas of Peculiar Galaxies by Arp (1966). These studies inspired pioneering numerical simulations (e.g. Toomre \& Toomre (1972)) which first suggested that elliptical galaxies may form from the coalescence of disks, thereby changing the status of mergers from rare exotica to fundamental agents for galaxy building. The significance of mergers and accretions has only grown in importance since then, as hierarchical cosmological mod-

${ }^{1}$ Department of Astronomy \& Astrophysics, University of Toronto, 50 St. George Street, Toronto, ON, M5S 3H4 Canada

2 Institute for Astronomy, University of Edinburgh, Blackford Hill, Edinburgh, EH9 3HJ United Kingdom els have come to the forefront (e.g. White \& Frenk (1991)). The primary process for galaxy growth in these models is the merger and accretion of dark matter halos. Since baryonic material traces the dark matter (albeit imperfectly), the visible signatures of this mechanism are an important observable useful for testing the current cosmological paradigm.

The properties of tidal features resulting from mergers and accretions depend on many factors such as the relative masses of the interacting systems, the geometry of the encounter and the gravitational potential. In cases where mergers are of systems with roughly equal masses, relatively high surface brightness features are expected to form, and some of these are quite well understood. For example, Mihos et al. (1998) show that for a given mass ratio the main parameter governing the length and kinematics of tidal tails is simply the shape of the potential, with the strength of the perturbation being relatively independent of the 
encounter speed. Another class of features that are fairly well understood are the 'shell' systems seen in elliptical galaxies (e.g. Malin \& Carter 1983). It is likely that the azimuthal distribution of shells probes the geometry of the merger event, so that plunging mergers result in radially organized shells, while high angular momentum mergers result in shells distributed quasi-randomly in azimuth (e.g. Dupraz \& Combes 1986; Hernquist \& Quinn 1988). Unfortunately, most mergers in the Universe are not of equal mass galaxies, and most tidal features are not as easily visible, nor as well understood, as tails or shells.

Cosmological simulations driven by the desire to understand hierarchical galaxy formation naturally focus on the more common minor mergers and accretions and the remnant substructure these leave behind (Bullock \& Johnston 2005; Johnston et al. 2008; Cooper et al. 2010). The statistical properties of this substructure contain an imprint of the merging history and nature of the progenitor galaxies. A fundamental prediction of hierarchical galaxy formation models in a $\Lambda$-dominated Cold Dark Matter cosmology (LCDM) is that all galaxies are surrounded by a vast and complex network of ultra-low-surface brightness filaments and streams. A few such streams and filaments have been discovered around nearby galaxies, including the Milky Way (e.g. Belokurov (2006)) and M31 (e.g. Ferguson et al. 2002; McConnachie et al. 2009), providing irrefutable evidence that some systems formed, at least in part, hierarchically. However, other galaxies scrutinised to faint depths fail to show such coherent debris (e.g. Barker et al. 2009, 2012; Bernard et al. 2012; Bailin et al. 2012) and it remains unclear if the number of discrete substructures around the galaxy population as a whole agrees with LCDM expectations. Bullock \& Johnston (2005) and others have pointed out that the bulk of the substructure resulting from accretion events is expected to have extremely low surface brightness, $30 \mathrm{mag} / \operatorname{arcsec}^{2}$ or fainter, which is well below the limit typically reached in modern imaging surveys. Thus, our current understanding of the statistical properties of very faint tidal debris is rather limited.

In between the relatively well-understood and clearly visible structures such as tails and shells, and the yet-undetected network of complex ultralow-surface brightness structures predicted to ex- ist fainter than $30 \mathrm{mag} / \operatorname{arcsec}^{2}$, lies the middle ground of tidal structures that are clearly detected but whose demographics are poorly characterized. This middle ground represents the main focus of this paper, whose goal is to present a catalog of such structures. The ubiquity and origin of features in this surface brightness realm $\left(\sim 26-28 \mathrm{mag} / \operatorname{arcsec}^{2}\right)$ are surprisingly poorly understood but are of direct relevance for understanding a variety of processes associated with the growth of galaxies. Previous attempts to quantify the overall statistics of galaxy substructures have reported a wide range in the fraction of galaxies which exhibit features that are likely to be tidal in origin. Table 1 presents a summary of published results. The lack of agreement almost certainly reflects the range in survey depths and the different galaxy selection criteria adopted. The widest-area surveys (such as those based on data from the Sloan Survey) are fairly shallow and have only modest angular resolution. Smaller studies, such as van Dokkum (2005) and Tal et al. (2009), go deeper, sometimes probing RMS variations in the sky down to $\sim 28 \mathrm{mag} / \operatorname{arcsec}^{2}$ by stacking multiple bands together. In general, the deepest investigations focus on sub-populations, such as early-type galaxies or those existing in dense environments, which appear to show statistically significant differences in their fine structure fractions relative to the total galaxy population (van Dokkum 2005; Nair \& Abraham 2010; Adams et al. 2012).

The statistical characteristics of faint tidal substructures are surprisingly poorly understood. The lack of concordance in Table 1 highlights the need for a better understanding of the frequency and nature of tidal debris, including how these vary as a function of galaxy stellar population, mass and environment. This is the main focus of this paper, the goal of which is to provide a uniform catalog of tidal structures, grouped logically into descriptive forms. It is tempting to seek a design language for describing mergers based on computer-based classification techniques (e.g. Abraham et al. 1996; Abraham, van den Bergh \& Nair 2003). Ultimately, that would seem the be the best way forward, but at present the stateof-the-art remains visual classification 1 . The hu-

\footnotetext{
${ }^{1}$ Assuming data volume and classification time are not fac-
} 
TABLE 1

OVERVIEW OF FAINT SUBSTRUCTURE FROM PUBLISHED SURVEYS

\begin{tabular}{|c|c|c|c|c|c|}
\hline Investigation & $\mathrm{N}$ & Dataset & Selection & $\begin{array}{c}\text { SB limit } \\
{\left[\mathrm{mag} / \operatorname{arcsec}^{2}\right]}\end{array}$ & Summary \\
\hline This paper & 1781 & CFHTLS-Wide & $\begin{array}{l}r^{\prime}<17 \\
0.04<z<0.2 \\
\mathrm{M}_{r}^{\prime}<-19.3\end{array}$ & $\begin{array}{l}g_{A B}^{\prime} \sim 27.7 \\
\left(g^{\prime} r^{\prime} i^{\prime}\right)_{A B}{ }^{\mathrm{a}} \sim 27.3\end{array}$ & $\begin{array}{l}\text { Strong: } 17.6 \% \\
\text { Weak: } 25.2 \%\end{array}$ \\
\hline Adams et al. 2012 & 3551 & CFHT MENeaCS & $\begin{array}{l}\text { Cluster ETGs } \\
0.04<z<0.15 \\
\mathrm{M}_{r}^{\prime}<-20\end{array}$ & $r_{A B}^{\prime} \sim 26.5$ & $3 \%$ \\
\hline Sheen et al. 2012 & 273 & CTIO Mosaic II & $\begin{array}{l}\text { Cluster ETGs } \\
\mathrm{z} \lesssim 0.1 \\
\mathrm{M}_{r}^{\prime}<-20\end{array}$ & $r_{A B}^{\prime} \sim 30$ & $\begin{array}{l}\text { Total Sample: } 25 \% \\
\text { Bulge-dominated: } 38 \%\end{array}$ \\
\hline Kim et al. 2012 & 65 & $S^{4} G$ & ETGs & {$[3.6 \mu]_{A B} \sim 26.5$} & $17 \%$ \\
\hline Miskolczi et al. 2011 & 474 & SDSS DR7 & $\begin{array}{l}\text { Edge-on disks } \\
>2^{\prime} \text { diameter }\end{array}$ & $\left(g^{\prime} r^{\prime} i^{\prime}\right)_{A B}{ }^{\mathrm{a}} \sim 26^{\mathrm{b}}$ & $\begin{array}{l}\text { Strong: } 6 \% \\
\text { Weak: } 19 \%\end{array}$ \\
\hline Nair \& Abraham 2010 & 14034 & SDSS DR4 & $\begin{array}{l}g^{\prime}<16 \\
0.01<z<0.1\end{array}$ & $g_{A B}^{\prime} \sim 26.5$ & $7 \%$ \\
\hline \begin{tabular}{|l|l} 
Bridge et al. 2010 \\
\end{tabular} & 23854 & CFHTLS-Deep & $\begin{array}{l}\mathrm{i}_{\text {vega }}<21.9 \\
0.1<\mathrm{z}<1.2 \\
\mathrm{M}_{\star}>10^{9.5} \mathrm{M}_{\odot}\end{array}$ & $g_{\text {vega }}^{\prime}<29^{\mathrm{c}}$ & $\begin{array}{l}4.3 \%(\mathrm{z}=0.3) \\
19 \%(\mathrm{z}=1)\end{array}$ \\
\hline Tal et al. 2009 & 55 & $\operatorname{SMARTS}(1 \mathrm{~m})$ & $\begin{array}{l}\text { Ellipticals } \\
M_{B}<-20 \\
15<D_{L}<50 \mathrm{Mpc}\end{array}$ & $V_{\text {vega }} \sim 27.7$ & $73 \%$ \\
\hline \begin{tabular}{|l|l|} 
van Dokkum 2005 \\
\end{tabular} & 126 & $\begin{array}{l}\text { MUSYC }+ \\
\text { NOAO Deep-Wide }\end{array}$ & $\begin{array}{l}\text { ETGs } \\
\mathrm{R}<17 \\
0.04<z<0.2\end{array}$ & $\left(B V R_{A B}{ }^{\mathrm{a}}, B V I A B^{\mathrm{a}}\right) \sim 28$ & $\begin{array}{l}\text { Total sample: } 53 \% \\
\text { Bulge dominated: } 71 \%\end{array}$ \\
\hline Schweizer \& Seitzer 1988 & 74 & KPNO $0.9 \mathrm{~m}$ & $\mathrm{E} / \mathrm{SO}$ & IIIaJ $\sim 26.5$ & $\begin{array}{l}\text { Strong: } 16 \% \\
\text { Weak: }>50 \%\end{array}$ \\
\hline$\frac{\text { Malin \& Carter }}{\text { Maann \& Carter }}$ & $\begin{array}{c}327 \\
73\end{array}$ & $\begin{array}{l}\text { UK Schmidt } \\
\text { UK Schmidt }\end{array}$ & $\begin{array}{l}\text { E/SO } \\
\text { E/SO (isolated) }\end{array}$ & $\begin{array}{l}\text { IIIaJ } \sim 26.5 \\
\text { IIIAJ } \sim 26.5\end{array}$ & $\begin{array}{l}5.8 \% \text { (Shells only) } \\
16.5 \% \text { (Shells only) }\end{array}$ \\
\hline
\end{tabular}

${ }^{\mathrm{a}}$ Stacked.

$\mathrm{b}^{\mathrm{b}}$ Detections decline after this surface brightness, but individual features were detected down to nearly $28 \mathrm{mag} / \mathrm{arcsec}^{2}$.

${ }^{\mathrm{c}}$ Cosmological dimming at the mid-redshift point of their sample $(\mathrm{z}=0.65)$ is $2.2 \mathrm{mag} / \mathrm{arcsec}^{2}$, considerably higher than any other entry (e.g. $0.4 \mathrm{mag} / \operatorname{arcsec}^{2}$ for of our sample's mid-redshift point of $\mathrm{z}=0.1$ ). 
man eye-brain system has a remarkable sensitivity to faint, unique features, many of which escape automatic classification using presently available techniques (e.g. see the discussion in Adams et al. 2012). In devising categories for describing tidal features one should seek to assign structures to groups with physical relevance, but it is important to acknowledge from the outset that faint tidal debris may have a variety of origins. With the exception of tails (and possibly shells) it is not presently possible to definitively associate most forms of tidal debris with a particular type of interaction (e.g. major vs. minor mergers, plunging vs tangential orbits, grazing encounters etc.) based on morphology alone. We agree with the viewpoint espoused by Kormendy (1982), who noted that

"... morphology is more generally a 'soft' science, which is best viewed as preparation for more quantitative work. Its most important use may be to provide a list of specific questions which provide direction for this work."

Nevertheless, it seems reasonable to expect that some classification categories are more insightful than others. For example, Tal et al. (2009) suggests that diffuse structures surrounding earlytype galaxies may mostly be the product of gasfree ('dry') merging events, so it seems worthwhile to try to note the existence of such features when trying to understand the demographics of tidal structures. Therefore, in the present paper we categorize structures in descriptive ways that we hope will prove meaningful in future investigations. But we emphasize that our primary goal is a descriptive census of faint debris, regardless of origin, and defer to future work any serious attempt to interpret these structures.

To place the data upon which the present paper is based in some context, we note that the most comprehensive visual catalog of faint galactic features is currently that given in Nair \& Abraham (2010), which is based on data from the Sloan Digital Sky Survey (SDSS). The SDSS images are fairly uniform, but also relatively shallow and their typical image quality (median r-band seeing of 1.43 arcsec) is not up to the standards of the best

tors. ground-based observatories. Therefore the data in the present paper explores tidal features in galaxies at redshifts similar to that in Nair \& Abraham (2010), but probing to substantially deeper surface brightness levels using data with better image quality (median $r^{\prime}$-band seeing of 0.81 arcsec).

All magnitudes used in this paper are based on the AB system unless otherwise noted. All cosmological calculations assume a flat dark energydominated cosmology with $\Omega_{\mathrm{M}}=0.3, \Omega_{\Lambda}=0.7$ and $H_{0}=70 \mathrm{~km} \mathrm{~s}^{-1} \mathrm{Mpc}^{-1}$.

\section{The Data}

\subsection{CFHTLS-Wide}

The Canada-France-Hawaii Telescope Legacy Survey (CFHTLS) is the product of 450 nights of observations on CFHT from 2003-2009 using the MegaCam one square degree visible-wavelength imager (Boulade 2003). The survey was split into three parts: Very Wide (a shallow survey intended to be most useful for investigations of Kuiperbelt objects), Wide (optimized for studies of weak lensing), and Deep (optimized for synoptic investigations of distant supernovae over a relatively small area). In this paper we focus solely on the intermediate-depth data from Wide component of the survey (hereafter referred to as CFHTLS-W). This survey covers approximately 170 square degrees of the sky in four separate patches ranging in size from 25 to 72 square degrees. This paper focuses on CFHTLS-W instead of the deep synoptic survey because the large area of CFHTLS-W allows us to define a sizeable sample of nearby objects probed with sufficient spatial resolution in the rest-frame to allow the detection of thin features. Tidally disturbed galaxies at high- $z$ in the deep synoptic survey have already been investigated by Bridge, Carlberg \& Sullivan (2010), and in $\S 4$ of this paper we will compare our low-redshift results to their high-redshift results.

The CFHTLS-W survey is based on data obtained through five filters $\left(u^{*}, g^{\prime}, r^{\prime}, i^{\prime}, z^{\prime}\right)$ with the Wide survey total exposure time being approximately one hour per filter per field. The four wide fields were centered at the following J2000 coordinates: RA 02:18:00, Dec -07:00:00 (W1); RA 08:54:00, Dec -04:15:00 (W2); RA 14:17:54, Dec $+54: 30: 31$ (W3); and RA 22:13:18, Dec +01:19:00 (W4). Our analysis is based on publicly-available 

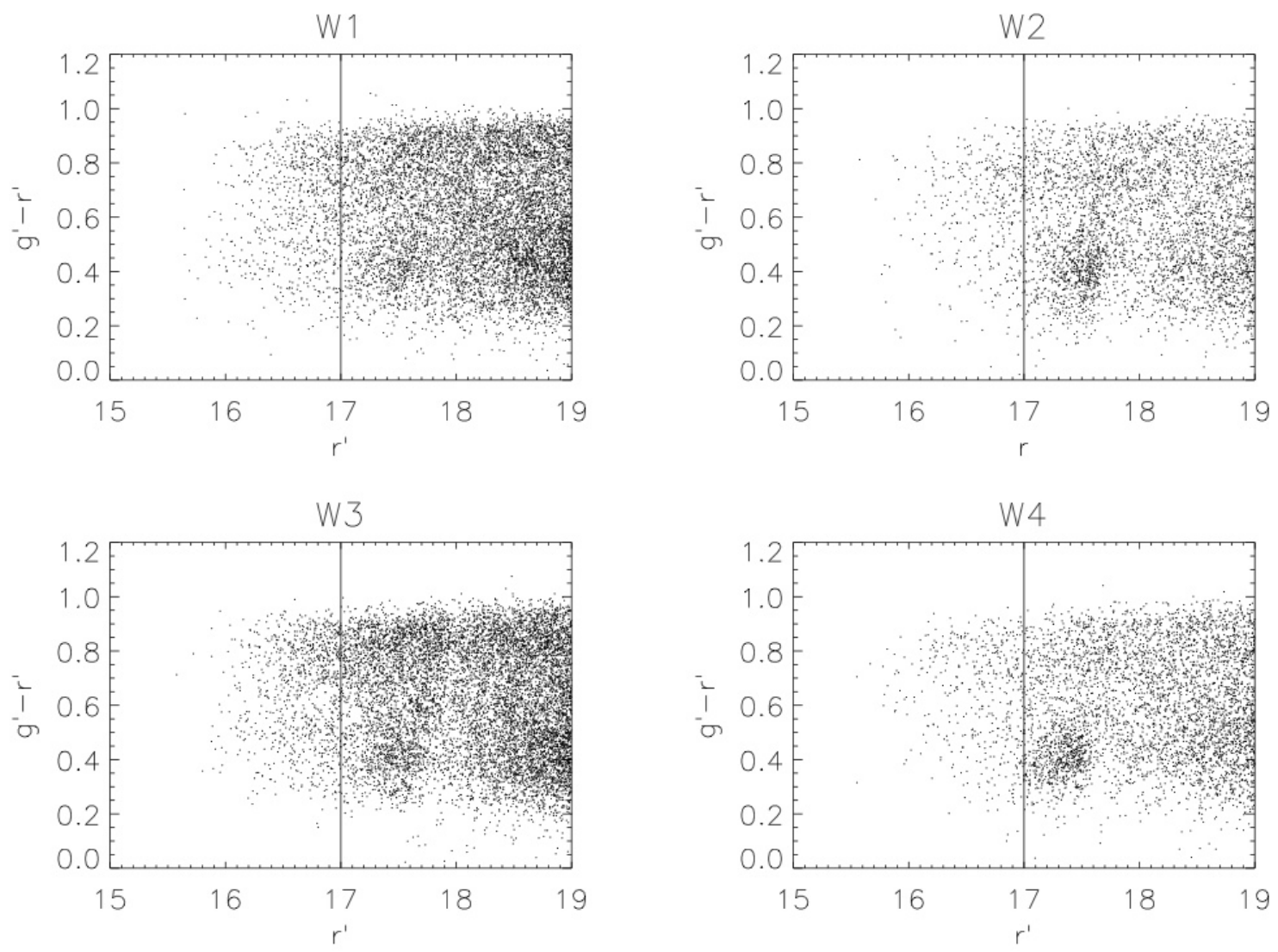

Fig. 1.- Colour-magnitude plots of galaxies in the four CFHTLS wide fields selected to be between the redshifts of 0.04 and 0.2 . We define our sample as galaxies with an $15.5<r^{\prime}<17$ mag. No initial cuts were made based on colour or morphology. Our sample is defined by points left of the vertical line at $r^{\prime}=17$ in each graph. After unusable thumbnail images are removed we are left with a sample of 1781 galaxies to be visually examined for streams. The cloud of points located from $17<r^{\prime}<18$ mag and $0.2<\left(g^{\prime}-r^{\prime}\right)<0.4$ appear to be stars that were incorrectly labelled as galaxies in the photo-z catalog. No automated process was found for removing such stars in our sample so they were removed manually during the inspection process.

image stacks provided by the MegaPipe pipeline (Gwyn 2008 ${ }^{2}$, to whom the reader is referred for details on the data reduction. Spectroscopic redshifts were unavailable for most galaxies in the sample, so publicly-available photometric redshifts provided by Coupon et al. (2009) were used instead. When needed, K-corrections were applied to the data using the program detailed in Chilingarian et al. (2010).

\footnotetext{
${ }^{2}$ http://www3.cadc-ccda.hia-iha.nrccnrc.gc.ca/community/CFHTLS-SG/docs/cfhtls.html
}

\subsection{Sample Selection}

Objects were selected for inspection by filtering the photometric redshift catalog to restrict consideration to objects in the redshift range $0.04<$ $z<0.2$. This range overlaps with that of Nair \& Abraham (2010) and allows for a direct comparison with van Dokkum (2005). An upper magnitude limit of $r^{\prime}=17 \mathrm{mag}$ was imposed in order to limit the sample size to a manageable number for visual inspection. A lower magnitude cutoff was imposed at $r^{\prime}=15.5 \mathrm{mag}$ because below this limit stars misidentified as galaxies begin to outnumber 


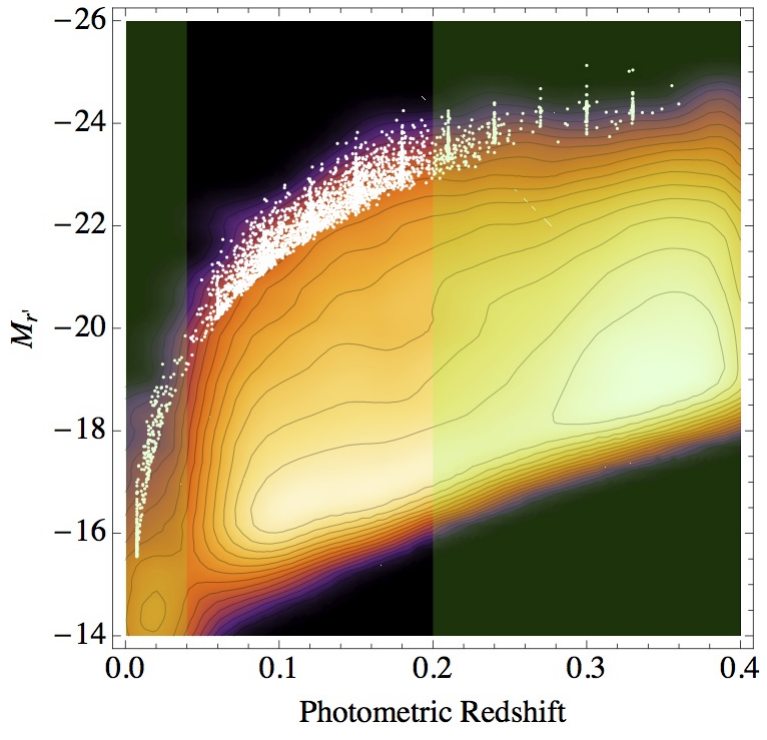

Fig. 2.- Absolute magnitude as a function of photometric redshift for galaxies which are brighter than the apparent magnitude limit of our sample (white points) superposed on the distribution for the full CFHT Legacy Survey sample out to $z=0.4$, shown as a smoothed density histogram (with logarithmically-spaced contours). Redshift cuts $(z<0.04$ and $z>0.2)$ used to excise very nearby and distant galaxies are shown as translucent green bands. Note how the final sample probes mainly the bright end of the galaxy distribution. See text for details.

galaxies in the photometric redshift catalog. The implications of these cuts are illustrated in Figure 1. which shows a color-magnitude diagram with included objects lying to the left of the vertical cut in each panel. (Note that objects failing to meet the photometric redshift or the bright magnitude cuts have already been removed in this figure). After removal of image artifacts (e.g. ghost images from bright stars, segments of diffraction spikes), misidentified stars, and otherwise unusable images (e.g., fields that are too crowded with foreground stars) 1781 galaxies remained in our sample. Our sample is heavily biased toward bright systems, as shown in Figure 2, This diagram compares the absolute magnitude as a function of redshift for our sample against the corresponding distribution for the full CFHT Legacy Survey. Most of the sample lies in the range $-23<M_{r^{\prime}}<-20$ mag. Our low-redshift cut eliminates most under-luminous galaxies, so the faintest galaxy in our sample has an absolute magnitude of $M_{r^{\prime}}=-19.3$ mag ${ }^{3}$. On the other hand, the absolute magnitudes of objects in our sample of typical of those of brighter galaxies seen at high redshifts, so our dataset is wellsuited for comparisons made against these. The typical half-light radii for our galaxies is $2-6$ arcsec.

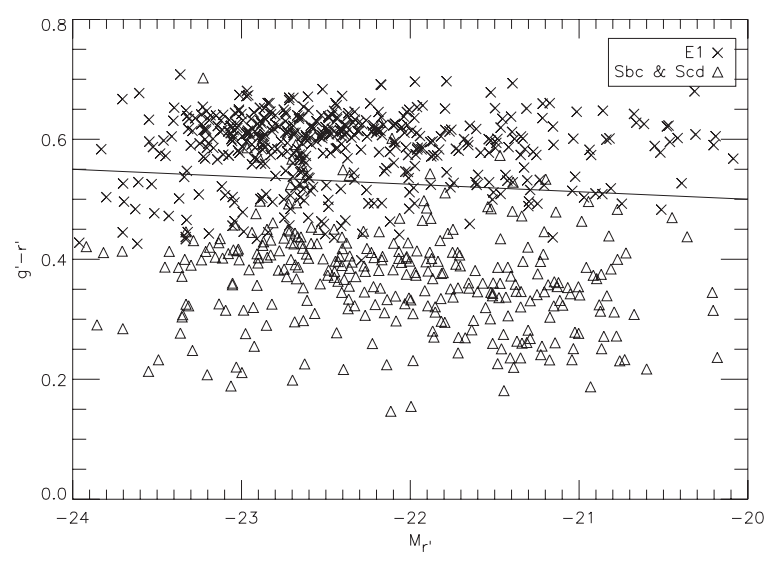

Fig. 3.- Colour-magnitude diagram for galaxies in the CFHTLS-W1 field. The line shown corresponds to $\left(g^{\prime}-r^{\prime}\right)=-0.0125 r^{\prime}+0.25$. As described in the text, this line is used to divide the total sample into red and blue sub-populations, corresponding to systems on the 'red sequence' and the 'blue cloud'. Plot symbols are keyed to galaxy templates used to determine photometric redshifts by Coupon et al. (2009). Galaxies identified by these authors as being best fit by the E1 template are plotted with crosses, while those best fit by Sbc and Scd templates are plotted with triangles. The corresponding plots for the three other CFHTLS wide fields (not shown) closely resemble this one. Over the entire sample, approximately $86 \%$ of red sequence galaxies were best fit by an E1 template while $84 \%$ of blue cloud galaxies were best fit by an Sbc or Scd template.

As will be shown below, the statistical properties of the tidally-disturbed galaxy sample are quite strongly dependent on rest-frame colour. It is therefore useful to subdivide the sample into red

\footnotetext{
${ }^{3}$ Note that Figure 2 clearly shows evidence for some discreteness in the photometric redshifts in the Coupon et al. (2009) catalog, but this is at a level that is insignificant for our purposes.
} 
and blue sub-populations from the outset. Subpopulations are defined based on position in the $\left(g^{\prime}-r^{\prime}\right)$ vs. $r^{\prime}$ colour-magnitude diagram, using the following line to subdivide the galaxy population:

$$
\left(g^{\prime}-r^{\prime}\right)=-0.0125 r^{\prime}+0.25
$$

This line was defined by us to to discriminate between systems on the red sequence and blue cloud (Bell et al. 2004: Strateva et al. 2001). As shown in Figure 3 this line does an excellent job of subdividing galaxies based on fits to the galaxy templates used by Coupon et al. (2009) to determine the photometric redshifts for the sample. Because of the correlations between rest-frame color and Hubble stage, our color-based subdivision of the galaxy population results in a coarse morphological segregation as well. For example, $86 \%$ of all red sequence galaxies were best fit with an E1 template by Coupon et al. (2009), while $84 \%$ of all blue sequence galaxies were best fit by an Sbc or Scd template.

\subsection{Visual Inspection}

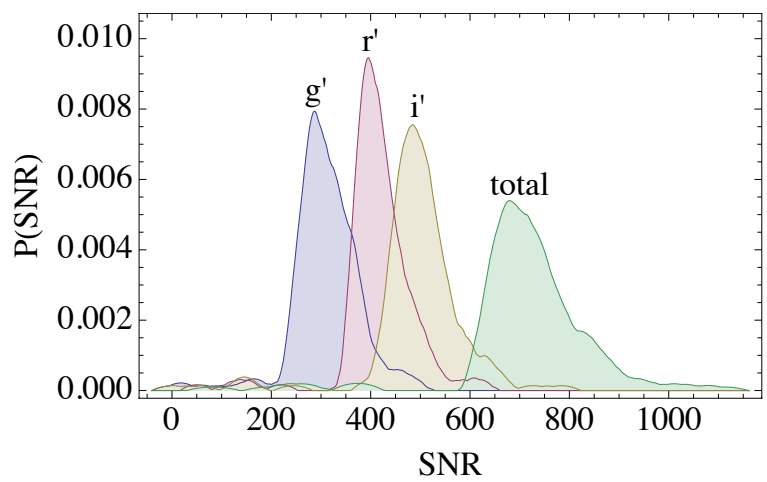

Fig. 4.- Smoothed probability density functions showing the signal-to-noise ratios in the $g^{\prime}, r^{\prime}$ and $i^{\prime}$-band images and the co-added $g^{\prime}+r^{\prime}+i^{\prime}$ stack. Histogram smoothing is done using an Epanechnikov kernel density estimator. See text for details.

Thumbnail images in the $g^{\prime}, r^{\prime}$ and $i^{\prime}$ band of each galaxy were cut out of the CHFTLSWide fields and stacked together (i.e. summed) to increase contrast. The relatively low signal-tonoise $u^{*}$ and $z^{\prime}$ images were not co-added, as the gain in signal-to-noise obtained was deemed insufficient. For each thumbnail the signal-to-noise level was estimated by using SExtractor to compute the ratio of the isophotal flux to the error on the isophotal flux reported by the progran 4 (Bertin \& Arnouts 1996). Distributions of SNR for the thumbnails are shown in Figure 4. As expected, the total signal to noise of the galaxy in the stacked frame is well-approximated by simply adding the individual signal-to-noises of the filtered data in quadrature. The median signalto-noise ratio of a stacked galaxy in our sample is 712 , which is a remarkably close match to the mean signal-to-noise ratio of galaxies in the Nair \& Abraham (2010) catalog of morphological classifications from the SDSS (712 for $g^{\prime}$-band, 735 for $r^{\prime}$ band, 592 for $i^{\prime}$-band). Note that Nair \& Abraham (2010) did not stack images in different filter bands to increase signal-to-noise.

To determine the limiting depth of the data, 40 square apertures, each with an area of 1.2 square arcsec were placed in empty regions of 120 thumbnails chosen to equally represent all four wide fields. The RMS variations of the total enclosed flux within these apertures, for each thumbnail image, were then computed to serve as a representation of the sky noise for each thumbnail image. Results are shown in Figure 5. In the $g^{\prime}$ band we find a mean limiting surface brightness of $\sim 27.7$ $\mathrm{mag} / \mathrm{acsec}^{2}$ (which corresponds to $\sim 0.5 \%$ of the g-band night sky brightness) with a standard deviation of $\sim 0.5 \mathrm{mag} / \mathrm{sq}$ arcsec. This provides a good representation of the limiting depth to which we can detect features in this band on small scales, although in practise large features can be detected to even lower surface brightness limits. Note that the limiting surface brightness of the $g^{\prime}$ data is fainter than the limiting surface brightness of the data obtained in redder bands (Figure 5), but the signal-to-noise (Figure 4 ) level of the redder bands is higher. This is simply due to the zero points underlying astronomical magnitude systems, which are biased toward blue objects (Vega in the traditional system, or flat spectrum sources in $F_{\nu}$ in the AB system).

The thumbnails were visually inspected in the viewing program DS9 (Joye \& Mandel 2003) to allow interactive manipulation of the screen transfer

\footnotetext{
${ }^{4}$ In other words, FLUX_ISO/FLUXERR_ISO.
} 

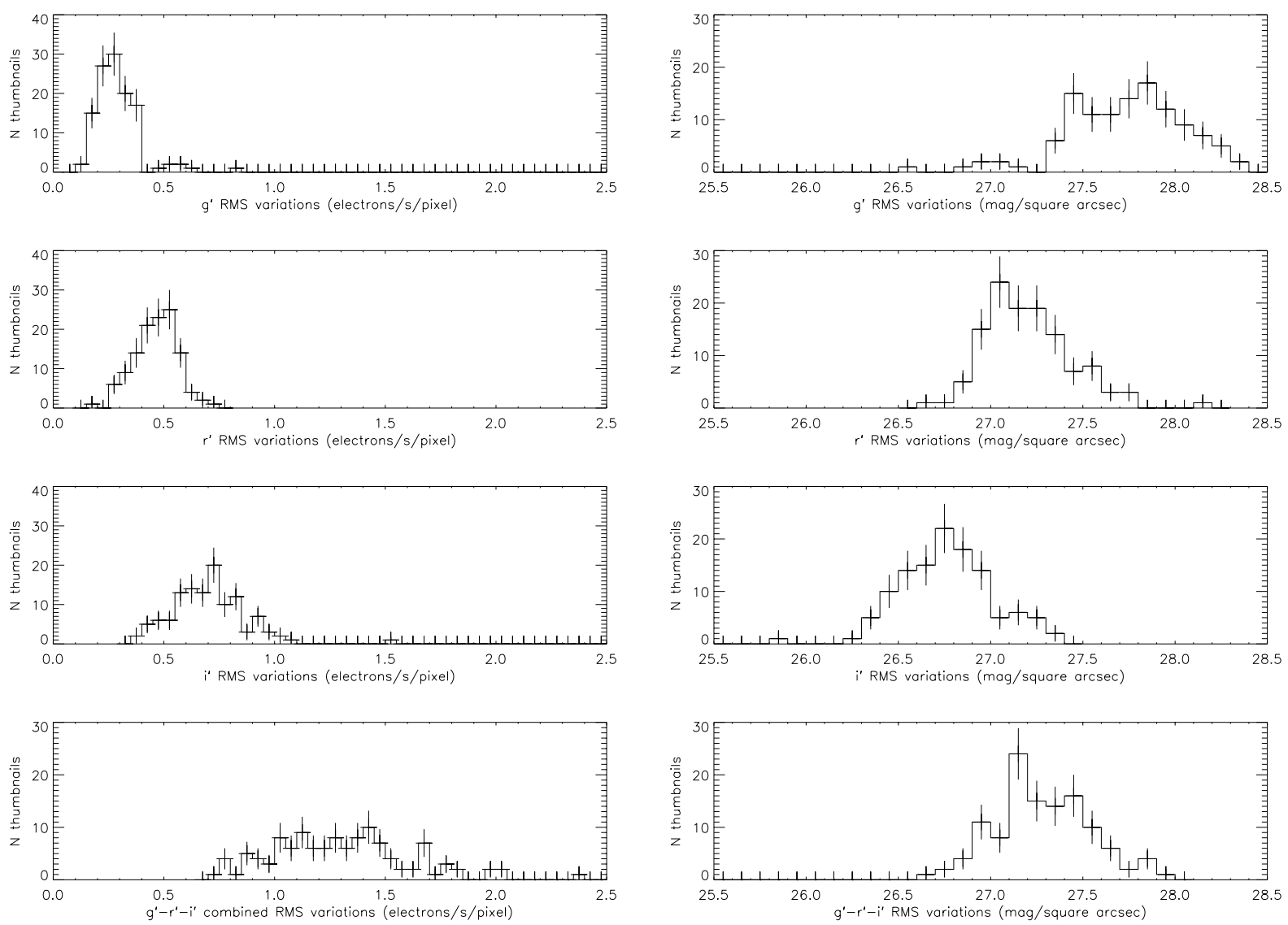

Fig. 5.- Histogram of the RMS variations due to sky noise estimated from 120 thumbnail images of separate galaxies representing all four wide fields. Noise for each of the thumbnails was found by placing 401.2 square arcsecond apertures in each image, and then computing the standard deviation of the mean. The left-hand column presents data in electron/s/pixel, while the right-hand column shows the corresponding values in $\mathrm{mag} / \operatorname{arcsec}^{2}$. Since the $g^{\prime}+r^{\prime}+i^{\prime}$ stack does not correspond to a standard photometric band the definition of an $\mathrm{AB}$ magnitude is used to backtrack an equivalent zero point. Rows correspond to $g^{\prime}, r^{\prime}, i^{\prime}$, and stacked $g^{\prime}+r^{\prime}+i^{\prime}$. See text for details.

function, and the images were grouped into one of five bins corresponding to the inspector's confidence that a tidal feature was present. The five confidence bins are defined in Table 2, Confidence bin 4 corresponds to the inspector being certain that the galaxy in question contains a tidal feature. Confidence level 3 corresponds to a galaxy that is very likely contains a feature, with something around $75 \%$ certainty. In our opinion it is reasonable to group confidence bins three and four together to define a sample with strong indications of tidal features. Confidence level 2 indicates a $50 \%$ likelihood that a tidal feature is present.
Treatment of such systems is naturally problematic, and it is left up to the reader to determine whether these objects should considered to be detections or non-detections. In the present paper we treat them as non-detections. Confidence level 1 corresponds to there being a hint that a tidal feature is present, while level 0 indicates no visible tidal features (or, alternatively, strong confidence that no features are present down to the surface brightness levels probed by our data). Studies of the reliability of morphological classifications (e.g. Naim et al. 1995) show that the internal consistency of a well-trained individual's mor- 
phological classifications tends to be higher than the observer-observer consistency of morphological classifications made by similarly well-trained people working on the same data. Therefore it is often unwise to have a few individuals visually classify the same sample and then 'vote' to determine a final classification. However, in the present instance, there is no well-defined classification scheme (such as the Hubble system) in place which describes tidal features. Under the circumstances, it was felt that some preliminary intercomparison of individual classifications by separate individuals was in order. Therefore 138 galaxies were examined by all three authors in order to verify the reproducibility of the most basic classification attempted, which is simply the observer's confidence that the galaxy being inspected shows evidence for any form of tidal disturbance. Excellent agreement was found on systems classified as 3 or 4 (high confidence that tidal features exist) and on systems classed as 0 (high confidence that no features exist). As might be anticipated, the only significant disagreements occurs for systems classified as 1 and 2 (objects with possible hints of tidal features). Encouraged by these comparisons, all 1781 galaxies were then inspected by a single classifier (Atkinson) in order to ensure consistency.

Tidal features were classified broadly into six categories: (1) streams; (2) arms; (3) linear features; (4) diffuse fans; (5) shells; and (6) miscellaneous diffuse structure. Stacked thumbnail images of all six types of features are shown in Figure 6, while examples of the six features at varying confidence levels are depicted in Figure 7. These features are not mutually exclusive. In fact, they should be viewed as a basis set of features from which descriptions of more elaborate tidal structures can be composed. For example, an 'umbrella-like' structure is seen in a number of galaxies, and this can be described as a combination of a 'linear feature' and a 'shell' (a boolean 'AND' operation can be used on the electronic caltalog to do this efficiently). In other cases features can be combined to yield broader more familiar classifications using a boolean 'OR'; for example, a set of galaxies with 'tails' can be constructed by extracting all systems with either 'streams' or 'arms' or 'linear features'. While it is our hope that our basis features might be indicative of different types of interactions (e.g., broad

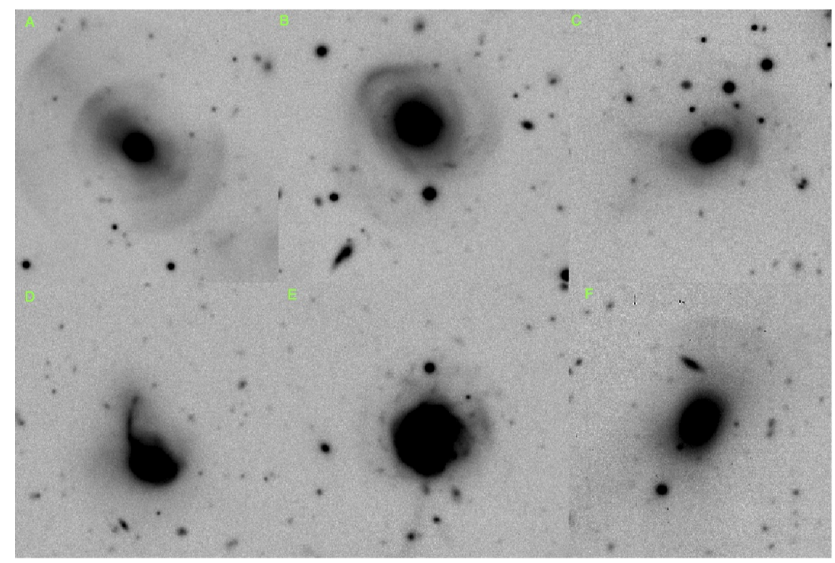

Fig. 6.- Examples of the six different categories of tidal disturbances used to classify objects in this paper. (a) Shells surrounding a galaxy, (b) A stream (visible in the lower part of the image), (c) Miscellaneous diffuse structure, (d) An arm, (e) A linear feature (visible at the bottom of the image), (f) Broad fans of diffuse light. Each of the six thumbnails is approximately $56 \times 56$ arcsec.

fans may result from dry mergers, as claimed by van Dokkum 2005), it is beyond the scope of our paper to test this. As noted earlier, our aim here is to provide a basic catalog which upon which future investigations of such questions can be based.

\section{Results}

The central results of this paper are summarized in Table 3 and shown in Figures 8 11 . Each of these figures is presented in a way that allows the reader to compare the fraction of systems showing tidal disturbances as a function of rest-frame colour. Table 4 lists the classifications and ancillary data for all galaxies in our sample 5 .

We find that $11.6 \pm 0.8 \%$ of galaxies in our sample show tidal features at the highest confidence level (confidence level 4). This fraction rises to $17.6 \pm 1.0 \%$ if systems with with confidence level three (objects with 'probable' features) are included, and to $25.2 \pm 1.2 \%$ if systems with confidence level two ('possible' features) are also in-

\footnotetext{
${ }^{5} \mathrm{An}$ extract from this table is given in the print version of this paper. The full version appears in the electronic version.
} 
TABLE 2

DeteCtion Classes

\begin{tabular}{cl}
\hline \hline Confidence level & \multicolumn{1}{c}{ Definition } \\
\hline 4 & Certain detection of a tidal feature \\
3 & Probable detection of a tidal feature (over 75 percent certain) \\
2 & Possible detection of a tidal feature (around 50 percent certain) \\
1 & Hint of a potential tidal feature. Very uncertain. \\
0 & No evidence for tidal features seen \\
\hline
\end{tabular}

TABLE 3

Summary of Tidal Feature Detections

\begin{tabular}{|c|c|c|c|c|c|c|}
\hline \multirow[b]{2}{*}{ Confidence } & \multicolumn{2}{|c|}{ Total Sample } & \multicolumn{2}{|c|}{ Red Galaxies } & \multicolumn{2}{|c|}{ Blue Galaxies } \\
\hline & Number & $\%$ & Number & $\%$ & Number & $\%$ \\
\hline 4 & 207 & $11.6 \pm 0.8$ & 153 & $15.4 \pm 1.2$ & 54 & $6.9 \pm 0.9$ \\
\hline 3 & 107 & $6.0 \pm 0.6$ & 70 & $7.0 \pm 0.8$ & 37 & $4.7 \pm 0.8$ \\
\hline 2 & 136 & $7.6 \pm 0.7$ & 79 & $7.9 \pm 0.9$ & 57 & $7.2 \pm 1.0$ \\
\hline 1 & 201 & $11.3 \pm 0.8$ & 103 & $10.4 \pm 1.0$ & 98 & $12.4 \pm 1.3$ \\
\hline 0 & 1130 & $63.4 \pm 1.9$ & 589 & $59.3 \pm 2.4$ & 541 & $68.7 \pm 3.0$ \\
\hline \# Galaxies & \multicolumn{2}{|c|}{1781} & \multicolumn{2}{|c|}{994} & \multicolumn{2}{|c|}{787} \\
\hline
\end{tabular}




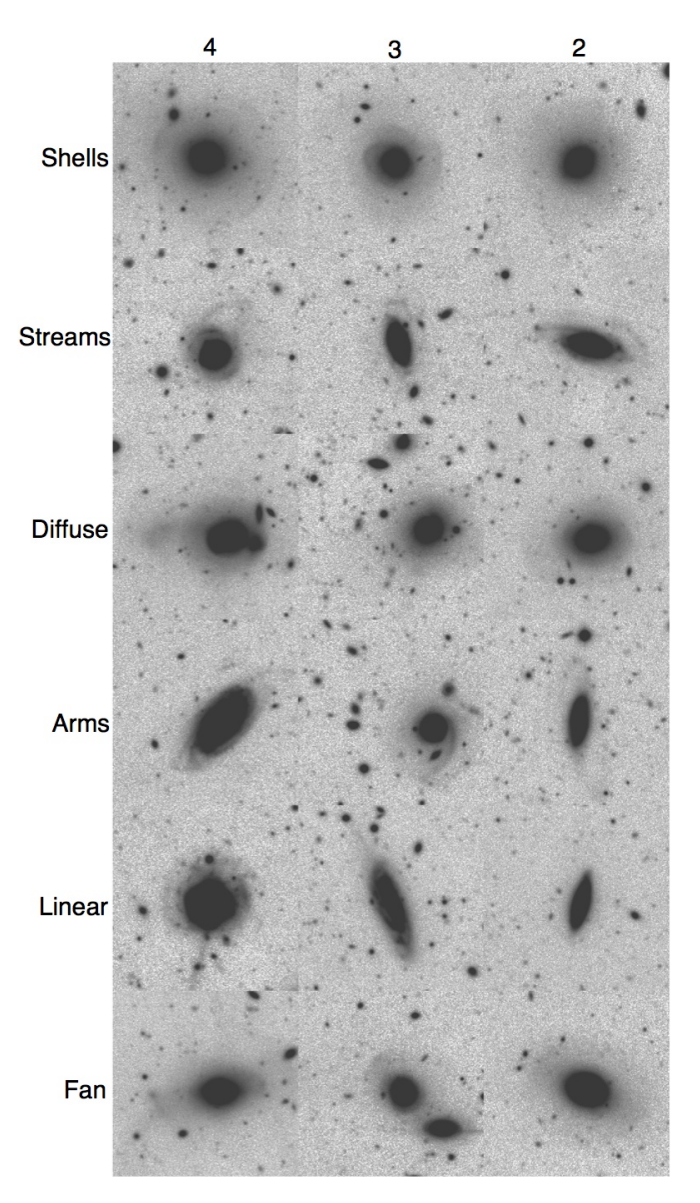

Fig. 7.- Examples of all six classifications of tidal features (top to bottom: shells, streams, miscellaneous diffuse structure, arms, linear features and fans) in three different confidence bins with decreasing confidence from left to right (bins four, three and two. Columns correspond to the confidences levels (defined in Table 2 ) that the tidal feature exists. Each individual thumbnail is approximately $56 \times 56$ arcsec.

cluded. In the Discussion section of this paper we will consider why these fractions are well below the $50-75 \%$ of systems reported to have tidal features by van Dokkum (2005) and Tal et al. (2009).

Figure 8 depicts our overall findings, while Figure 9 emphasizes that chromatic effects play a role in defining the probability that a given galaxy will show tidal disturbances. Interestingly, we can now

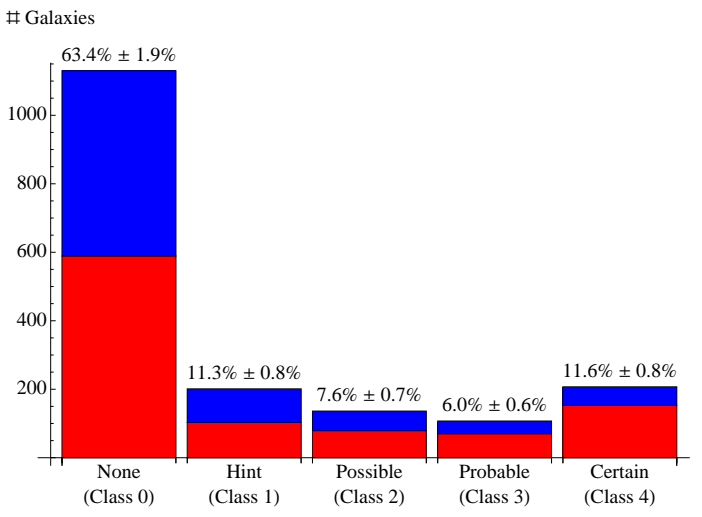

Fig. 8.- Distribution of detection classes in the CFHTLS-Wide dataset. Each histogram bin is labeled by its fractional contribution to the total galaxy population, and each bin is subdivided into red sequence and blue cloud populations.

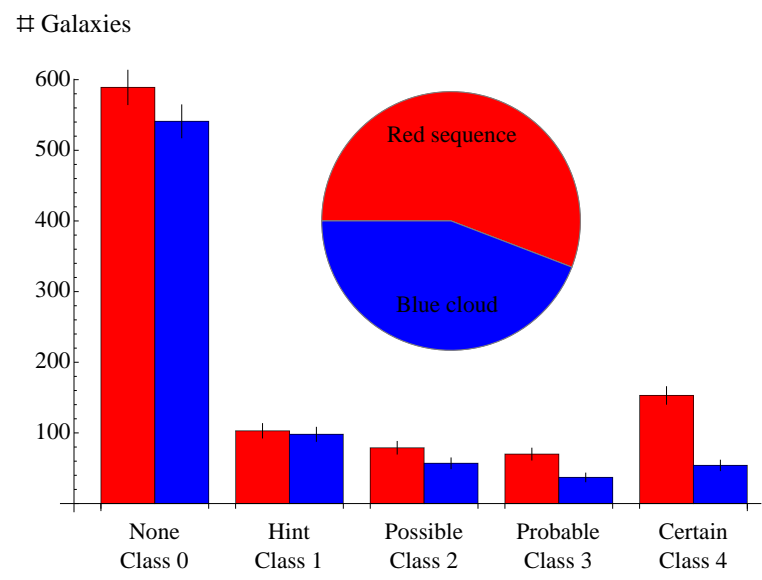

Fig. 9.- Distribution of feature classifications. Each class of tidal feature is subdivided into separate bins of galaxy colour.

also show that the nature of the tidal disturbances is a strong function of colour. Red galaxies are over twice as likely to show signs of tidal disturbance compared to blue galaxies. This effect is most striking in the high-confidence bin (confidence levels three and four), becoming somewhat diluted in the low confidence bins, presumably as real tidal structures become diluted by spurious false detections.

Figures 10 and 11 illustrate the incidence of tidal disturbances as a function of feature class. Figure 10 shows the distribution of features seen 


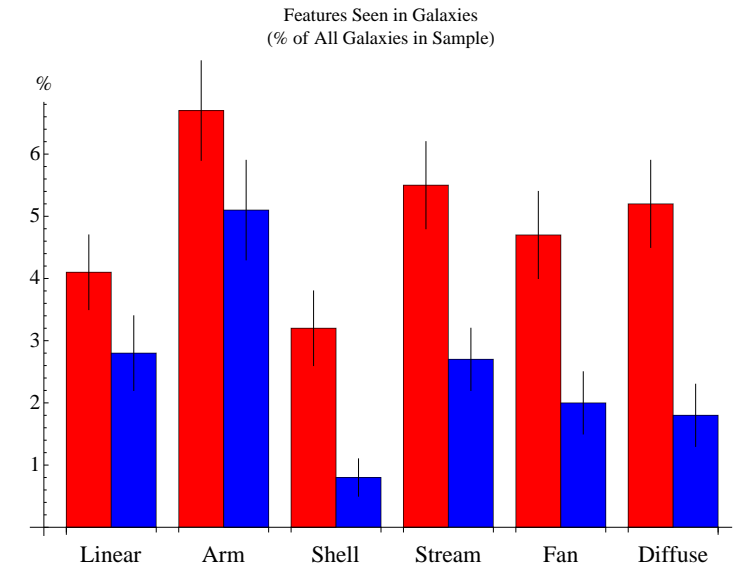

Fig. 10.- Distribution of high-confidence (levels 3 and 4) detections of tidal features subdivided by galaxy colour. Red bars correspond to galaxies in the red sequence, while blue bars correspond to galaxies in the blue cloud. Percentages shown correspond to fractions relative to the total number of galaxies of that colour.

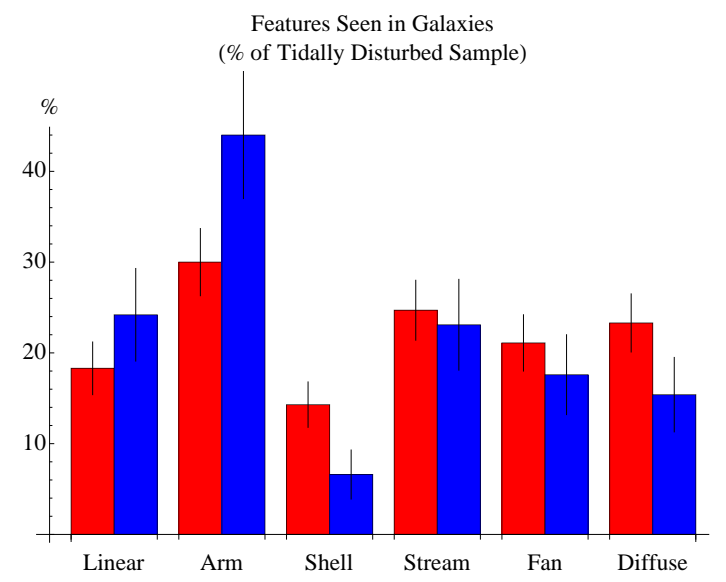

Fig. 11.- Distribution of tidal features subdivided by galaxy colour. Red histogram bars correspond to red galaxies, while blue histogram bars correspond to blue galaxies. Percentages shown correspond to fractions relative to the number of high-confidence tidally disturbed galaxies (class 3 and 4) of that colour.

with high confidence (class 3 and 4 ) relative to the total galaxy sample, while Figure 11 shows the distribution of features relative to the population of tidally disturbed galaxies of each particular color ${ }^{6}$ These two figures are clearly closely related, but they make different points, so we will describe each of them in turn.

Figure 10 shows that every type of tidal disturbance is more likely to be found in a red galaxy than in a blue galaxy. In some ways this seems unsurprising - some features, such as shells, are generally associated with elliptical galaxies, and our analysis confirms that shells are far more likely to appear in red galaxies than in blue galaxies (unsurprising, since a major merger leading to an elliptical would likely destroy a disk; c.f. Hernquist \& Spergel 1992). However, other post-merger debris features, such as bridges (generally classified in the present paper as 'arms' or 'diffuse structures' which connects two galaxies) and tails (generally grouped into the 'arm' and 'linear' classes), are generally associated with disks. For example, all the systems modelled in the classic paper by Toomre \& Toomre (1972) are blue star-forming objects 7 Our analysis shows that these sorts of features are also more common in red galaxies than in blue galaxies.

Our analysis indicates that all types of disturbances are more common in red galaxies than in blue galaxies, but that red and blue populations are markedly different in the diversity of their tidal features. Red galaxies show a plethora of structures, with roughly the same fraction of red galaxies exhibiting 'arms', 'streams', 'fans' and 'miscellaneous diffuse structures'. Amongst red galaxies, the fraction of galaxies exhibiting each of these classes is statistically indistinguishable at the $90 \%$ confidence level. Blue galaxies, on the other hand, are much more likely to exhibit 'arms' than any other class of structure.

This point can be seen somewhat more clearly by inspection of Figure 11, which shows our data normalized to the number of tidally disturbed galaxies of each colour. We see from this figure that, in both red and blue populations, 'arms' are the most common tidal feature and 'shells' are the least common tidal feature. Amongst disturbed blue galaxies, $45 \%$ show tidal arms, while shells

\footnotetext{
${ }^{6}$ Note that tidal features are not mutually exclusive, so summing the values in Figure 11 exceeds $100 \%$.

${ }^{7}$ Arp 295 , M51 + NGC51995 (the 'whirlpool'), NGC4676 (the 'mice') and NGC4038/9 (the 'antennae').
} 
are only seen in $6 \%$ of this population. The $7: 1$ difference in the occurrence rate for these features amongst blue galaxies is in sharp contrast to the barely 2:1 difference for these features amongst red galaxies. Evidently tidally-disturbed red galaxies tend to exhibit a richer variety of dynamical structures than do blue galaxies.

\section{Discussion}

\subsection{Comparison with Previous Work}

Table 1 summarizes the basic results from a number of earlier investigations into the frequency of tidal structures seen in nearby galaxies. As we have already noted, there is little consensus. Setting aside the fact that some surveys span a broad redshift range over which evolutionary effects may be important (e.g. Bridge et al. 2010), we attribute the lack of homogeneity in the local galaxy surveys to two main factors: (1) the surveys vary in depth, and (2) the surveys probe a range of galaxy populations, depending on selection criteria.

The inhomogeneous depth of the surveys presented in Table 1 has important implications both in terms of the apparent magnitude limit of the galaxies probed, and the limiting surface brightness of the datasets. The former limitation can, to some extent, be addressed by our investigation. In the next section we will show how the mix of tidal features seen in galaxy populations varies with the luminosity (or stellar mass) of the galaxies being surveyed. On the other hand, the latter factor is (to some extent) ameliorated by the fact that it is presently difficult to undertake any study which probes to a surface brightness below about $0.5 \%$ of the sky, owing to systematic effects. The implication is that even very deep investigations are generally confined to limiting surface brightnesses in a band between $27 \mathrm{mag} / \operatorname{arcsec}^{2}$ and 28 $\mathrm{mag} / \operatorname{arcsec}^{2}$ in the $\mathrm{V}$-band. Whether the abundance of tidal features changes greatly within this band is an open question, but there are hints that it does. For example, we noted in the previous section that the fraction of red galaxies in our study showing evidence for tidal features is a factor of 2-3 lower than found by van Dokkum (2005) and Tal et al. (2009), who investigated the structure of elliptical galaxies at similar luminosity and redshift to those studied in the present paper and found that $50-75 \%$ of galaxies contain such features. This contrasts with the $22.4 \pm 1.5 \%$ of red galaxies showing high-confidence (class 3 or 4 ) features in our investigation. It appears that van Dokkum (2005) probes down to a limiting surface brightness that is about $0.8 \mathrm{mag} / \operatorname{arcsec}^{2}$ deeper than our own suggesting that the majority of tidal features in early-type galaxies are seen at surface brightness near (or below) $28 \mathrm{mag} / \operatorname{arcsec}^{2}$.

Recent work by Kim et al. (2012) finds significantly fewer instances of structure (17\%) in Early Type Galaxies in the Spitzer Survey of Stellar Structure in Galaxies, which is more consistent with the results of this work. However, it should be noted that a small number of previously identified features were not detected in galaxies within their sample, including at least one instance of shells far enough away from the parent galaxy that they were thought to be outside the field of view of Kim et al. (2012).

Perhaps the most similar work to our own in concept is that of Miskolczi et al. (2011) who found that only $6 \%$ of their sample had distinct features and $19 \%$ showed faint indications of features. Our higher detection rates may arise in part from differences in the classification method, as well as the more obvious differences in depth and seeing. For example, broad features such as fans and shells are included in our classification system but not theirs. Regardless, comparison with future studies by this group with larger sample sizes should prove interesting.

As has been noted, a number of the surveys presented in Table 1 focus on systems selected in a particular way. In some cases, selection makes it impossible for fair comparisons to be made. For example, it is difficult to make a direct comparison between our work and the sample provided by Martinez-Delgado et al. (2010). These authors find a wealth of substructure in a handful of nearby galaxies, but many of their targets are well-known systems selected in advance for prominent substructure and in no way can be considered a statistical sample. In other cases, more detailed comparisons are possible. It is particularly interesting to compare our work with that of Malin \& Carter (1983), who focused on shells in elliptical galaxies. We find that $3.2 \pm 0.5 \%$ of the red galaxies in our study have shells (based on systems with confidence levels 3 and 4), compared 

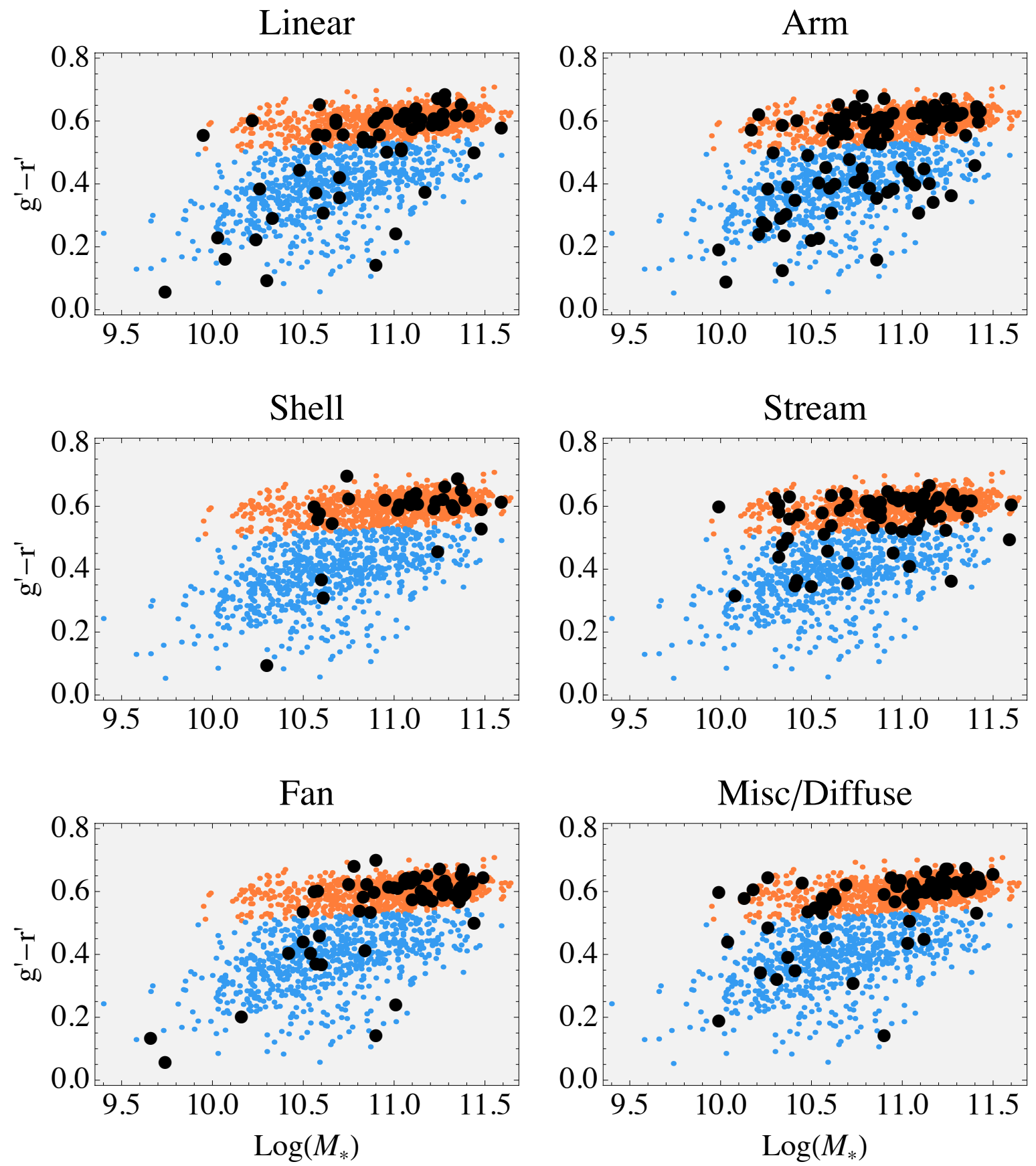

Fig. 12.- Distribution of tidal features as a function of stellar mass and rest-frame $g^{\prime}-r^{\prime}$ color. Each panel shows the total galaxy population subdivided by color. Plot symbols denote red sequence galaxies as red points, and blue cloud galaxies as blue points. In addition, each panel isolates sub-populations exhibiting specific tidal features identified by the panel labels. Starting at the top left and running clockwise, the panels show the distribution of galaxies with linear structures, arms, streams, miscellaneous/diffuse structures, fans, and shells. In each panel the galaxies exhibiting these features are plotted as large black disks. It is clear that some of the trends encapsulated by Figures 8 and 9 are strong functions of stellar mass. See text for details. 
to $5.8 \%$ of theirs. It is difficult to directly compare the limiting depth of CCD-based studies to photographic investigations, but we suspect that the limiting depths of the studies are comparable, and that most of the discrepancy stems from the manner in which we have subdivided tidal features into six categories. It is likely that some features Malin \& Carter (1983) classified as shells would be classified fans in the present paper (see Figure 6 for the general similarity of the features). Around $8 \%$ of our red galaxy sample exhibit shells or fans. It is also interesting that Malin \& Carter (1983) find shell frequency increases threefold when samples are restricted to isolated galaxies. More recent studies seem to disagree on the role of the environment with Adams et al. (2012) and Sheen et al. (2012) finding very different debris frequencies in rich galaxy clusters. This may be due to differing survey depths, however.

\subsection{Galaxy Mass-Dependence of Tidal Features}

As noted in the previous section, it is interesting to consider whether the visibility of the tidal features identified in our sample is dependent on the stellar mass of the tidally disturbed galaxy. Figure 12 presents the distribution of galaxies with strong (confidence class 3 and 4) tidal features as a function of stellar mass and rest-frame $g^{\prime}-r^{\prime}$ color. Stellar masses were estimated from the restframe luminosities and colours using the methodology described in Bell et al. (2003), assuming a Kroupa initial mass function (Kroupa et al. 1993). Each panel in Figure 12 corresponds to a separate tidal feature, and the galaxy population are colour-coded according to position within the blue cloud (blue points) or red sequence (red points). Galaxies exhibiting the tidal features isolated in each panel are shown as large black points.

Although our sample does not span a large range in stellar mass, a number of interesting conclusions can be drawn from Figure 12. As has already been noted, 'linear' features occur most frequently in red systems, but the figure reveals a strong mass dependence in the visibility of such features on the red sequence, with a bias toward the most massive galaxies. Linear features (as well as shells and fans; see below) occur much more frequently in galaxies with stellar masses $>10^{10.5} \mathrm{M}_{\odot}$.
The small number of linear features seen in galaxies in the blue cloud do not appear to show any mass dependence. Systems containing 'shells' also show a strong mass dependence, although these features are sufficiently rare in blue cloud galaxies that we would be unable to characterize any mass dependence in these objects even if it existed. Systems containing 'fans' appear to show trends very similar to those for systems containing 'linear' features. Overall, our data lends support to the claim by van Dokkum (2005) that diffuse fan-like features might be associated with dry merging activity, since they are found most frequently in massive red sequence galaxies. Although, it is worth noting that shells seem an even better tracer of dry mergers, and that linear features appear to be about as effective as fans for tracing tidal interactions on the red sequence.

Interestingly, not all tidal features exhibit evidence for a dependence on stellar mass. There is little evidence for any mass dependence in the 'arm' classifications in either red or blue galaxies. The main impression that emerges from Figure 10 for this class of features is simply a reinforcement of the conclusion from Figure 9 that tidal arms are the dominant form of tidal feature seen in systems on the blue cloud. There also appears to be little evidence for mass dependence in 'streams' in either the red or blue populations. If this class of feature traces minor accretion events, then the implication is that these occur at a similar rate in both red and blue systems. Finally, there appears to be a hint of bimodality in the mass dependence of features classified as 'miscellaneous/diffuse', which suggests that, befitting its name, this category contains at least two independent types of structures.

In an investigation of on-going mergers out to $z=0.7$, Chou et al. (2011) show that at blue mergers (with colours typical of the blue cloud) are far more common than red mergers (with colours typical of the red sequence), but our findings here for the more local universe indicate that faint debris is more common in red systems. If the preponderance of tidal features in red galaxies is indicative of dry mergers then, taken together with the Chou et al. (2011) result, this suggests that either the accretion rate onto red galaxies has been increasing in the very recent past or that tidal features in red galaxies are longer-lasting. It is difficult to 
draw firm conclusions because Chou et al. (2011) explores much further down the faint end of the local luminosity function than we do. The presence of tidal features shows a fairly strong mass dependence, and it is clear from Figure 12 that the $r^{\prime}=17$ mag cut strongly biases our sample toward the bright end of the luminosity function, which is dominated by early-type systems. An investigation which probes the demographics of tidal structures in fainter galaxy populations would likely prove interesting.

\section{Conclusions}

Using the wide-field component of the CanadaFrance-Hawaii Telescope Legacy Survey, we have analysed the incidence of tidal disturbances in 1781 luminous $\left(M_{r^{\prime}}<-19.3 \mathrm{mag}\right)$ galaxies in the magnitude range $15.5<r^{\prime}<17 \mathrm{mag}$ and in the redshift range $0.04<z<0.2$. We present our results in the form of a catalog that we hope will form the basis for future investigations into the nature and origin of low surface brightness debris around galaxies. Although we have classified tidal features according to morphology (e.g. streams, shells and tails), we do not attempt to interpret them in terms of their physical origin (e.g. post-merger debris versus arising from a minor accretion event). We find that around $12 \%$ of the galaxies in our sample show clear tidal features at the highest confidence level. This fraction rises to about $18 \%$ if we include systems with convincing albeit weaker tidal features, and to $26 \%$ if we include systems with more marginal features that may or may not be tidal in origin. These proportions are a strong function of rest-frame colour and of stellar mass. Linear features, shells, and fans are much more likely to occur in massive galaxies with stellar masses $>10^{10.5} \mathrm{M}_{\odot}$, and red galaxies are twice as likely to show tidal features than are blue galaxies. We discuss how our overall statistics compare to those reported in the literature and conclude that varying photometric depths and selection criteria can lead to significant variations in the derived debris frequencies.

Based on observations obtained with MegaPrime/ MegaCam, a joint project of CFHT and CEA (DAPNIA), at the Canada-France-Hawaii Telescope (CFHT) which is operated by the National
Research Council (NRC) of Canada, the Institut National des Science de l'Univers of the Centre National de la Recherche Scientifique (CNRS) of France, and the University of Hawaii. This wo is based in part on data products produced at the Canadian Astronomy Data Centre as part of the Canada-France-Hawaii Telescope Legacy Survey, a collaborative project of NRC and CNRS. This research made use of the "K-corrections calculator" service available at http://kcor.sai.msu.ru/, This research has also made use of SAOImage DS9, developed by Smithsonian Astrophysical Observatory

\section{REFERENCES}

Abraham, R. G., van den Bergh, S., Glazebrook, K., et al. 1996, ApJS, 107, 1

Abraham, R. G., van den Bergh, S., \& Nair, P. 2003, ApJ, 588, 218

Adams S. M., Zaritsky D., Sand D. J., Graham M. L., Bildfell C., Hoekstra H., Pritchet C., 2012, AJ, 144, 128

Arp, H. 1966, Pasadena: California Inst. Technology, 1966,

Bailin, J., Bell, E. F., Chappell, S. N., RadburnSmith, D. J. and de Jong, Roelof. S. 2011, ApJ, 736,24

Barker M. K., Ferguson A. M. N., Irwin M., Arimoto N., Jablonka P., 2009, AJ, 138, 1469

Barker, M. K., Ferguson, A. M. N., Irwin, M. J., Arimoto, N. and Jablonka, P. 2012, MNRAS, 419,1489

Bell, E., McIntosh, D., Katz, N and Weinberg, M. 2003, ApJS, 149, 289

Bell, E. et al. 2004, ApJ, 608, 752

Belokurov, V. et al. 2006, ApJ, 642, L137

Bernard E. J., Ferguson A. M. N., Barker M. K., Irwin M. J., Jablonka P., Arimoto N., 2012, MNRAS, 426, 3490

Bertin, E. and Arnouts, S. 1996, A\&AS, 117, 393

Boulade, O. et al. 2003, SPIE, 4841, 72 
Bridge, C., Carlberg, R. and Sullivan, M. 2010, ApJ, 709, 1067

Bullock, J. S. and Johnston, K. V. 2005, ApJ, 635, 931

Chilingarian, I., Melchior, A.-L. and Zolotukhin, I. 2010, MNRAS, 405, 1409

Chou, Richard C. Y., Bridge, Carrie R., and Abraham, Roberto, G. 2011, AJ, 141, 87

Cooper, A. et al. 2010, MNRAS, 406, 744

Coupon et al. 2009, A\&A, 500, 981

van Dokkum, P. 2005, ApJ, 130, 2647

Dupraz, C., \& Combes, F. 1986, A\&A, 166, 53

Ferguson, A. M. N., Irwin, M. J., Ibata, R. A., Lewis, G. F., Tanvir, N. R., 2002, AJ, 124, 1452

Gwyn, S. 2008, PASP, 120, 212

Hernquist, L., \& Quinn, P. J. 1988, ApJ, 331, 682

Hernquist, L., \& Spergel, D. N. 1992, ApJ, 399, L117

Johnston, K. V., Bullock, J. S., Sharma, S., Font, A., Robertson, B. E., Leitner, S. N. 2008, ApJ, 689, 936

Joye, W. A. and Mandel, E. 2003, ASPC, 295, 489

Kim, T., et al. 2012, Preprint, arXiv:1204.3879v1

Kormendy, J. 1982, Saas-Fee Advanced Course 12: Morphology and Dynamics of Galaxies, 113

Kroupa, P., Tout, C. A., Gilmore, G. 1993, MNRAS, 262, 545

Malin, D. and Carter, D. 1983, ApJ, 274, 534

Martinez-Delgado, D. et al. 2010, AJ, 140, 962

Mathewson, D.S. Cleary, M.N., Murray, J.D. 1974, ApJ, 120, 291

McConnachie A. W., et al., 2009, Nature, 461, 66

Mihos, J.C., Harding, P., Feldmeier, J. and Morrison, H. 2005 ApJ, 631L, 41

Mihos, J. C., Dubinski, J., \& Hernquist, L. 1998, ApJ, 494, 183
Miscolczi, A., Bomans, D. and Dettmar, R. 2011, A\&A, 536, 66

Naim, A., Lahav, O., Buta, R. J., et al. 1995, MNRAS, 274, 1107

Nair, P. and Abraham, R. 2010, ApJS, 186, 427

Peng, C., Ho, L., Impey, C. and Rix, H. 2010 AJ139, 2097

Schweizer, F. and Seitzer, P. 1988, ApJ, 328, 88

Sheen Y.-K., Yi S. K., Ree C. H., Lee J., 2012, ApJS, 202, 8

Slater, C. T., Harding, P. and Mihos, C. 2009, PASP, 121, 1267

Strateva, I. et al. 2001, AJ, 122, 1861

Tal, T., van Dokkum, P., Nelan, J. and Benzanson, R. 2009, AJ, 138, 1417

Toomre, A., \& Toomre, J. 1972, ApJ, 178, 623

Vorontsov-Velyaminov, B. A. 1959, Atlas and catalog of interacting galaxies (1959)

Vorontsov-Velyaminov, B. A. 1977, A\&AS, 28, 1

White, S. D. M. and Frenk, C. S. 1991, ApJ, 379, 52

This 2-column preprint was prepared with the AAS LATEX macros v5.2. 
TABLE 4

MASTER DATA TABLE ${ }^{\mathrm{a}}$

\begin{tabular}{|c|c|c|c|c|c|c|c|c|c|}
\hline ID & Confidence $^{\mathrm{b}}$ & Features $^{c}$ & $\begin{array}{c}\mathrm{RA} \\
{[\mathrm{J} 2000]}\end{array}$ & $\begin{array}{c}\text { Dec } \\
{[\mathrm{J} 2000]}\end{array}$ & $z_{p h o t}$ & $\begin{array}{c}\mathrm{M}_{r}^{\prime \mathrm{d}} \\
{[\mathrm{mag}]}\end{array}$ & $\begin{array}{c}\mathrm{M}_{\star}{ }^{\mathrm{e}} \\
{\left[\log \left(\mathrm{M}_{\odot}\right)\right]}\end{array}$ & $\begin{array}{c}\left(g^{\prime}-r^{\prime}\right)^{\mathrm{f}} \\
{[\mathrm{mag}]}\end{array}$ & Red/Blue ${ }^{g}$ \\
\hline W1-2 & 4 & $\mathrm{~L}, \mathrm{~A}, \mathrm{~F}$ & 30.690147 & -11.165118 & 0.147 & -22.38 & 11.06 & 0.623 & $\mathrm{R}$ \\
\hline W1-3 & 1 & - & 30.201271 & -11.144945 & 0.178 & -23.43 & 11.24 & 0.413 & B \\
\hline W1-4 & 0 & - & 30.391056 & -11.107487 & 0.182 & -23.71 & 11.36 & 0.414 & B \\
\hline W1-7 & 4 & $\mathrm{~L}, \mathrm{~S}, \mathrm{~A}$ & 30.196264 & -11.033447 & 0.120 & -22.10 & 10.95 & 0.623 & $\mathrm{R}$ \\
\hline W1-8 & 0 & - & 30.493235 & -11.019429 & 0.113 & -21.76 & 10.80 & 0.616 & $\mathrm{R}$ \\
\hline W1-9 & 0 & - & 30.513254 & -10.806742 & 0.111 & -21.88 & 10.62 & 0.412 & B \\
\hline W1-11 & 0 & - & 31.069776 & -10.696386 & 0.126 & -22.02 & 10.67 & 0.402 & B \\
\hline W1-13 & 0 & - & 30.596039 & -10.623502 & 0.152 & -22.71 & 10.94 & 0.400 & $\mathrm{~B}$ \\
\hline W1-17 & 4 & $\mathrm{~S}$ & 31.714083 & -11.228167 & 0.104 & -21.79 & 10.82 & 0.615 & $\mathrm{R}$ \\
\hline W1-18 & 2 & - & 31.970299 & -11.213195 & 0.163 & -22.55 & 10.69 & 0.235 & $\mathrm{~B}$ \\
\hline W1-19 & 4 & $\mathrm{H}$ & 31.412792 & -11.151110 & 0.120 & -22.38 & 11.02 & 0.586 & $\mathrm{R}$ \\
\hline W1-20 & 1 & - & 31.203613 & -11.146258 & 0.180 & -23.15 & 11.39 & 0.639 & $\mathrm{R}$ \\
\hline W1-21 & 0 & - & 31.808828 & -10.948963 & 0.150 & -22.84 & 11.29 & 0.660 & $\mathrm{R}$ \\
\hline W1-22 & 1 & - & 31.982681 & -10.953930 & 0.090 & -21.13 & 10.26 & 0.354 & B \\
\hline W1-25 & 0 & - & 32.125488 & -11.198564 & 0.180 & -23.03 & 11.32 & 0.626 & $\mathrm{R}$ \\
\hline W1-26 & 0 & - & 32.415287 & -11.132730 & 0.117 & -22.03 & 10.59 & 0.326 & $\mathrm{~B}$ \\
\hline W1-27 & 0 & - & 32.646156 & -11.124942 & 0.180 & -22.82 & 11.09 & 0.496 & B \\
\hline W1-29 & 4 & $\mathrm{~A}$ & 32.386871 & -11.023117 & 0.154 & -22.63 & 10.86 & 0.352 & $\mathrm{~B}$ \\
\hline W1-30 & 0 & - & 32.990086 & -10.963011 & 0.121 & -22.31 & 11.02 & 0.614 & $\mathrm{R}$ \\
\hline W1-31 & 0 & - & 32.767452 & -10.926092 & 0.147 & -22.51 & 10.93 & 0.459 & $\mathrm{~B}$ \\
\hline W1-32 & 1 & - & 32.871616 & -10.871783 & 0.096 & -22.27 & 10.71 & 0.345 & B \\
\hline W1-34 & 0 & - & 32.128754 & -10.664643 & 0.179 & -23.16 & 11.12 & 0.403 & $\mathrm{~B}$ \\
\hline W1-36 & 1 & - & 32.194176 & -10.582054 & 0.150 & -22.64 & 11.18 & 0.636 & $\mathrm{R}$ \\
\hline W1-37 & 2 & - & 32.136494 & -10.360971 & 0.150 & -22.43 & 11.09 & 0.634 & $\mathrm{R}$ \\
\hline W1-41 & 4 & M & 32.218201 & -10.266704 & 0.148 & -22.74 & 11.19 & 0.611 & $\mathrm{R}$ \\
\hline W1-42 & 4 & $\mathrm{~S}$ & 33.921970 & -11.095670 & 0.063 & -20.47 & 10.30 & 0.623 & $\mathrm{R}$ \\
\hline W1-45 & 0 & - & 33.566002 & -10.765550 & 0.136 & -22.41 & 11.04 & 0.592 & $\mathrm{R}$ \\
\hline W1-46 & 1 & - & 33.697021 & -10.732102 & 0.110 & -22.00 & 10.66 & 0.397 & B \\
\hline W1-47 & 1 & - & 33.428829 & -10.721240 & 0.180 & -23.32 & 11.45 & 0.639 & $\mathrm{R}$ \\
\hline W1-48 & 3 & $\mathrm{~A}$ & 33.135857 & -10.580472 & 0.099 & -21.53 & 10.62 & 0.528 & $\mathrm{R}$ \\
\hline W1-49 & 4 & $\mathrm{~S}, \mathrm{M}$ & 33.770798 & -10.468553 & 0.120 & -22.56 & 11.10 & 0.596 & $\mathrm{R}$ \\
\hline W1-50 & 3 & $\mathrm{~A}$ & 33.911705 & -10.416350 & 0.168 & -23.35 & 11.09 & 0.304 & $\mathrm{~B}$ \\
\hline W1-53 & 0 & - & 34.150101 & -11.225227 & 0.139 & -22.38 & 10.84 & 0.427 & $\mathrm{~B}$ \\
\hline W1-54 & 0 & - & 34.520470 & -11.128219 & 0.167 & -22.71 & 10.97 & 0.428 & $\mathrm{~B}$ \\
\hline W1-57 & 0 & - & 34.712013 & -10.842353 & 0.120 & -22.21 & 10.99 & 0.618 & $\mathrm{R}$ \\
\hline W1-58 & 3 & $\mathrm{~A}$ & 34.556000 & -10.782074 & 0.146 & -22.73 & 10.92 & 0.371 & $\mathrm{~B}$ \\
\hline W1-59 & 0 & - & 34.974277 & -10.764244 & 0.180 & -23.02 & 11.34 & 0.647 & $\mathrm{R}$ \\
\hline W1-60 & 3 & $\mathrm{~A}$ & 34.698021 & -10.600347 & 0.100 & -21.53 & 10.36 & 0.301 & B \\
\hline W1-62 & 2 & - & 35.200176 & -11.199566 & 0.148 & -22.33 & 10.95 & 0.544 & $\mathrm{R}$ \\
\hline W1-63 & 0 & - & 35.929020 & -11.171984 & 0.150 & -23.02 & 11.27 & 0.581 & $\mathrm{R}$ \\
\hline W1-64 & 3 & - & 35.778553 & -11.093374 & 0.179 & -23.53 & 11.41 & 0.525 & $\mathrm{~B}$ \\
\hline W1-66 & 4 & $\mathrm{~A}, \mathrm{M}$ & 35.650330 & -10.939105 & 0.122 & -22.38 & 11.06 & 0.622 & $\mathrm{R}$ \\
\hline W1-67 & 4 & $\mathrm{H}$ & 35.416416 & -10.864992 & 0.180 & -22.87 & 11.22 & 0.590 & $\mathrm{R}$ \\
\hline W1-68 & 0 & - & 35.235199 & -10.825553 & 0.148 & -22.32 & 10.76 & 0.371 & $\mathrm{~B}$ \\
\hline
\end{tabular}


TABLE 4-Continued

\begin{tabular}{|c|c|c|c|c|c|c|c|c|c|}
\hline ID & Confidence $^{\mathrm{b}}$ & Features $^{\mathrm{c}}$ & $\begin{array}{c}\text { RA } \\
{[\mathrm{J} 2000]}\end{array}$ & $\begin{array}{c}\text { Dec } \\
{[\mathrm{J} 2000]}\end{array}$ & $z_{p h o t}$ & $\begin{array}{c}\mathrm{M}_{r}^{\prime \mathrm{d}} \\
{[\mathrm{mag}]}\end{array}$ & $\begin{array}{c}\mathrm{M}_{\star}^{\mathrm{e}} \\
{\left[\log \left(\mathrm{M}_{\odot}\right)\right]}\end{array}$ & $\begin{array}{c}\left(g^{\prime}-r^{\prime}\right)^{\mathrm{f}} \\
{[\mathrm{mag}]}\end{array}$ & Red/Blue ${ }^{\mathrm{g}}$ \\
\hline W1-69 & 0 & - & 35.573376 & -10.795518 & 0.136 & -22.21 & 10.39 & 0.083 & B \\
\hline W1-71 & 0 & - & 35.441040 & -10.673303 & 0.184 & -23.19 & 11.35 & 0.593 & $\mathrm{R}$ \\
\hline W1-72 & 1 & - & 35.858185 & -10.487077 & 0.180 & -23.19 & 11.18 & 0.443 & $\mathrm{~B}$ \\
\hline W1-73 & 1 & - & 35.858341 & -10.480101 & 0.115 & -21.82 & 10.62 & 0.431 & B \\
\hline W1-74 & 0 & - & 35.084522 & -10.463946 & 0.110 & -21.82 & 10.47 & 0.297 & $\mathrm{~B}$ \\
\hline W1-75 & 1 & - & 35.383976 & -10.428904 & 0.157 & -22.58 & 11.14 & 0.619 & $\mathrm{R}$ \\
\hline W1-76 & 2 & - & 35.646030 & -10.419737 & 0.182 & -23.20 & 11.35 & 0.586 & $\mathrm{R}$ \\
\hline W1-79 & 1 & - & 35.107166 & -10.274105 & 0.162 & -22.99 & 11.11 & 0.446 & $\mathrm{~B}$ \\
\hline W1-80 & 0 & - & 36.192448 & -11.037560 & 0.135 & -22.48 & 10.95 & 0.488 & $\mathrm{~B}$ \\
\hline W1-81 & 1 & - & 36.751522 & -10.966722 & 0.090 & -21.29 & 10.23 & 0.268 & B \\
\hline W1-82 & 0 & - & 36.480877 & -10.924501 & 0.136 & -22.43 & 10.79 & 0.363 & $\mathrm{~B}$ \\
\hline W1-83 & 0 & - & 36.506718 & -10.863957 & 0.155 & -22.95 & 11.02 & 0.382 & $\mathrm{~B}$ \\
\hline W1-84 & 2 & - & 36.806564 & -10.775711 & 0.150 & -22.69 & 11.17 & 0.611 & $\mathrm{R}$ \\
\hline W1-85 & 4 & $\mathrm{~A}$ & 36.796375 & -10.761139 & 0.173 & -22.78 & 10.95 & 0.382 & B \\
\hline W1-87 & 0 & - & 36.753819 & -10.687791 & 0.161 & -22.64 & 11.08 & 0.547 & $\mathrm{R}$ \\
\hline W1-90 & 0 & - & 36.269184 & -10.614738 & 0.127 & -22.30 & 11.02 & 0.614 & $\mathrm{R}$ \\
\hline W1-91 & 0 & - & 36.255611 & -10.593030 & 0.075 & -21.02 & 10.13 & 0.278 & $\mathrm{~B}$ \\
\hline W1-92 & 0 & - & 36.755485 & -10.493033 & 0.155 & -22.66 & 11.11 & 0.565 & $\mathrm{R}$ \\
\hline W1-93 & 2 & - & 36.146633 & -10.478559 & 0.150 & -22.69 & 11.13 & 0.577 & $\mathrm{R}$ \\
\hline W1-95 & 3 & $\mathrm{~S}$ & 36.204315 & -10.464885 & 0.180 & -23.05 & 11.31 & 0.606 & $\mathrm{R}$ \\
\hline W1-96 & 1 & - & 36.370941 & -10.446504 & 0.076 & -20.85 & 10.06 & 0.272 & $\mathrm{~B}$ \\
\hline W1-97 & 2 & - & 36.408279 & -10.303560 & 0.070 & -20.84 & 10.42 & 0.600 & $\mathrm{R}$ \\
\hline W1-98 & 2 & - & 37.792419 & -11.126202 & 0.172 & -23.06 & 10.85 & 0.189 & B \\
\hline W1-99 & 0 & - & 37.685009 & -11.122371 & 0.123 & -21.82 & 10.30 & 0.144 & $\mathrm{~B}$ \\
\hline W1-100 & 0 & - & 37.452518 & -11.041182 & 0.180 & -23.07 & 11.35 & 0.632 & $\mathrm{R}$ \\
\hline W1-101 & 1 & - & 37.476299 & -10.962550 & 0.151 & -23.14 & 11.30 & 0.566 & $\mathrm{R}$ \\
\hline W1-102 & 0 & - & 37.457287 & -10.896633 & 0.149 & -22.47 & 11.05 & 0.577 & $\mathrm{R}$ \\
\hline W1-103 & 0 & - & 37.460041 & -10.823323 & 0.120 & -21.94 & 10.87 & 0.605 & $\mathrm{R}$ \\
\hline W1-104 & 0 & - & 37.025684 & -10.745133 & 0.180 & -22.87 & 11.23 & 0.605 & $\mathrm{R}$ \\
\hline W1-110 & 4 & $\mathrm{~L}, \mathrm{~S}$ & 37.223198 & -10.591873 & 0.128 & -22.22 & 10.70 & 0.354 & $\mathrm{~B}$ \\
\hline W1-111 & 0 & - & 37.241467 & -10.566697 & 0.148 & -22.70 & 10.71 & 0.199 & $\mathrm{~B}$ \\
\hline W1-112 & 0 & - & 37.670563 & -10.531555 & 0.120 & -21.93 & 10.84 & 0.583 & $\mathrm{R}$ \\
\hline W1-113 & 2 & - & 37.715500 & -10.368069 & 0.182 & -23.23 & 11.49 & 0.702 & $\mathrm{R}$ \\
\hline W1-114 & 0 & - & 38.727276 & -11.166331 & 0.150 & -23.00 & 11.33 & 0.642 & $\mathrm{R}$ \\
\hline W1-115 & 0 & - & 38.126999 & -11.066277 & 0.099 & -21.87 & 10.47 & 0.280 & $\mathrm{~B}$ \\
\hline W1-117 & 1 & - & 38.804420 & -11.023103 & 0.120 & -22.26 & 10.89 & 0.516 & $\mathrm{~B}$ \\
\hline W1-118 & 0 & - & 38.320705 & -11.016455 & 0.116 & -21.81 & 10.56 & 0.376 & B \\
\hline W1-120 & 2 & - & 38.449925 & -10.950802 & 0.149 & -22.67 & 11.03 & 0.494 & $\mathrm{~B}$ \\
\hline W1-122 & 0 & - & 38.525471 & -10.858728 & 0.043 & -19.82 & 9.67 & 0.300 & $\mathrm{~B}$ \\
\hline W1-123 & 0 & - & 37.933041 & -10.739057 & 0.150 & -22.41 & 11.05 & 0.605 & $\mathrm{R}$ \\
\hline W1-125 & 0 & - & 38.624039 & -10.501018 & 0.180 & -23.22 & 11.39 & 0.616 & $\mathrm{R}$ \\
\hline W1-126 & 4 & $\mathrm{~S}, \mathrm{~L}$ & 38.071007 & -10.471202 & 0.151 & -22.50 & 11.11 & 0.620 & $\mathrm{R}$ \\
\hline W1-127 & 2 & - & 38.575352 & -10.382453 & 0.139 & -22.44 & 11.04 & 0.584 & $\mathrm{R}$ \\
\hline W1-134 & 4 & $\mathrm{~L}$ & 30.613457 & -9.799909 & 0.157 & -23.35 & 11.17 & 0.372 & $\mathrm{~B}$ \\
\hline
\end{tabular}


TABLE 4-Continued

\begin{tabular}{|c|c|c|c|c|c|c|c|c|c|}
\hline ID & Confidence $^{\mathrm{b}}$ & Features $^{\mathrm{c}}$ & $\begin{array}{c}\text { RA } \\
{[\mathrm{J} 2000]}\end{array}$ & $\begin{array}{c}\text { Dec } \\
{[\mathrm{J} 2000]}\end{array}$ & $z_{p h o t}$ & $\begin{array}{c}\mathrm{M}_{r}^{\prime \mathrm{d}} \\
{[\mathrm{mag}]}\end{array}$ & $\begin{array}{c}\mathrm{M}_{\star}^{\mathrm{e}} \\
{\left[\log \left(\mathrm{M}_{\odot}\right)\right]}\end{array}$ & $\begin{array}{c}\left(g^{\prime}-r^{\prime}\right)^{\mathrm{f}} \\
{[\mathrm{mag}]}\end{array}$ & Red/Blue ${ }^{\mathrm{g}}$ \\
\hline W1-135 & 1 & - & 30.270990 & -9.787633 & 0.171 & -22.81 & 11.05 & 0.461 & B \\
\hline W1-136 & 0 & - & 30.594109 & -9.775151 & 0.122 & -22.28 & 10.82 & 0.447 & B \\
\hline W1-137 & 1 & - & 30.552790 & -9.671059 & 0.150 & -22.55 & 11.13 & 0.629 & $\mathrm{R}$ \\
\hline W1-139 & 1 & - & 30.535917 & -9.531606 & 0.141 & -22.21 & 10.85 & 0.493 & B \\
\hline W1-140 & 4 & - & 30.707821 & -9.336653 & 0.181 & -23.08 & 11.33 & 0.613 & $\mathrm{R}$ \\
\hline W1-141 & 0 & - & 31.767675 & -10.230257 & 0.150 & -22.59 & 11.14 & 0.622 & $\mathrm{R}$ \\
\hline W1-142 & 0 & - & 32.003788 & -10.192514 & 0.182 & -23.05 & 11.32 & 0.615 & $\mathrm{R}$ \\
\hline W1-143 & 3 & $\mathrm{H}$ & 31.730707 & -10.154305 & 0.152 & -22.52 & 11.09 & 0.602 & $\mathrm{R}$ \\
\hline W1-145 & 0 & - & 31.790983 & -10.093591 & 0.180 & -22.87 & 11.22 & 0.592 & $\mathrm{R}$ \\
\hline W1-148 & 4 & $\mathrm{~L}$ & 31.547184 & -9.947580 & 0.126 & -22.49 & 10.96 & 0.499 & $\mathrm{~B}$ \\
\hline W1-149 & 2 & - & 31.333336 & -9.935458 & 0.159 & -22.71 & 11.04 & 0.484 & $\mathrm{~B}$ \\
\hline W1-151 & 0 & - & 32.048481 & -9.898329 & 0.132 & -22.33 & 10.94 & 0.533 & $\mathrm{R}$ \\
\hline W1-152 & 0 & - & 31.227858 & -9.815267 & 0.150 & -22.69 & 11.17 & 0.607 & $\mathrm{R}$ \\
\hline W1-153 & 0 & - & 31.566692 & -9.807934 & 0.168 & -22.91 & 11.06 & 0.434 & B \\
\hline W1-154 & 0 & - & 31.557116 & -9.787653 & 0.090 & -21.71 & 10.75 & 0.581 & $\mathrm{R}$ \\
\hline W1-155 & 0 & - & 31.576050 & -9.666735 & 0.150 & -22.73 & 11.17 & 0.594 & $\mathrm{R}$ \\
\hline W1-156 & 4 & $\mathrm{~A}, \mathrm{~S}$ & 31.790985 & -9.564985 & 0.121 & -22.65 & 11.11 & 0.574 & $\mathrm{R}$ \\
\hline W1-157 & 3 & $\mathrm{~S}$ & 32.060642 & -9.546564 & 0.134 & -22.65 & 11.06 & 0.525 & $\mathrm{~B}$ \\
\hline W1-159 & 0 & - & 31.692123 & -9.438184 & 0.086 & -21.39 & 10.21 & 0.220 & $\mathrm{~B}$ \\
\hline W1-160 & 4 & $\mathrm{~L}$ & 31.720535 & -9.397388 & 0.180 & -22.88 & 11.21 & 0.583 & $\mathrm{R}$ \\
\hline W1-164 & 0 & - & 32.367489 & -10.114808 & 0.093 & -21.23 & 10.35 & 0.396 & $\mathrm{~B}$ \\
\hline W1-165 & 2 & - & 32.110931 & -10.109645 & 0.099 & -21.46 & 10.28 & 0.251 & $\mathrm{~B}$ \\
\hline W1-166 & 3 & $\mathrm{~S}$ & 32.816593 & -9.948530 & 0.133 & -22.10 & 10.88 & 0.561 & $\mathrm{R}$ \\
\hline W1-167 & 0 & - & 32.868648 & -9.949264 & 0.083 & -20.96 & 10.23 & 0.387 & $\mathrm{~B}$ \\
\hline W1-168 & 3 & $\mathrm{~F}$ & 32.871441 & -9.941854 & 0.150 & -22.79 & 11.18 & 0.584 & $\mathrm{R}$ \\
\hline W1-169 & 0 & - & 32.152912 & -9.937702 & 0.141 & -22.27 & 10.76 & 0.389 & $\mathrm{~B}$ \\
\hline W1-170 & 0 & - & 32.531841 & -9.908673 & 0.114 & -22.33 & 10.70 & 0.323 & $\mathrm{~B}$ \\
\hline W1-171 & 2 & - & 32.314884 & -9.784696 & 0.195 & -23.55 & 11.07 & 0.213 & B \\
\hline W1-174 & 0 & - & 32.157692 & -9.702569 & 0.137 & -22.40 & 10.81 & 0.390 & $\mathrm{~B}$ \\
\hline W1-175 & 0 & - & 33.062721 & -9.604751 & 0.100 & -21.52 & 10.69 & 0.598 & $\mathrm{R}$ \\
\hline W1-176 & 0 & - & 32.950943 & -9.591608 & 0.129 & -21.96 & 10.66 & 0.414 & $\mathrm{~B}$ \\
\hline W1-177 & 4 & $\mathrm{~A}$ & 32.297382 & -9.546334 & 0.092 & -21.61 & 10.74 & 0.617 & $\mathrm{R}$ \\
\hline W1-178 & 4 & $\mathrm{~A}$ & 32.297039 & -9.545559 & 0.092 & -21.38 & 10.64 & 0.601 & $\mathrm{R}$ \\
\hline W1-179 & 0 & - & 32.344719 & -9.524055 & 0.134 & -22.49 & 10.82 & 0.373 & $\mathrm{~B}$ \\
\hline W1-180 & 0 & - & 32.421181 & -9.526288 & 0.149 & -22.36 & 10.73 & 0.334 & $\mathrm{~B}$ \\
\hline W1-181 & 0 & - & 32.156177 & -9.475406 & 0.150 & -22.49 & 11.09 & 0.614 & $\mathrm{R}$ \\
\hline W1-182 & 1 & - & 33.442871 & -10.218962 & 0.095 & -21.34 & 10.62 & 0.605 & $\mathrm{R}$ \\
\hline W1-183 & 1 & - & 33.855614 & -10.179149 & 0.130 & -22.62 & 11.09 & 0.560 & $\mathrm{R}$ \\
\hline W1-184 & 0 & - & 33.697487 & -10.192449 & 0.149 & -22.41 & 10.95 & 0.517 & $\mathrm{~B}$ \\
\hline W1-185 & 2 & - & 33.166058 & -10.152111 & 0.102 & -21.87 & 10.61 & 0.401 & B \\
\hline W1-187 & 2 & - & 33.808041 & -10.090777 & 0.125 & -22.15 & 10.88 & 0.547 & $\mathrm{R}$ \\
\hline W1-188 & 2 & - & 33.166721 & -10.072129 & 0.119 & -22.91 & 11.11 & 0.476 & B \\
\hline W1-189 & 2 & - & 33.193256 & -10.045926 & 0.187 & -23.96 & 11.47 & 0.428 & $\mathrm{~B}$ \\
\hline W1-190 & 0 & - & 33.760208 & -10.066311 & 0.092 & -21.54 & 10.57 & 0.488 & $\mathrm{~B}$ \\
\hline
\end{tabular}


TABLE 4-Continued

\begin{tabular}{|c|c|c|c|c|c|c|c|c|c|}
\hline ID & Confidence $^{\mathrm{b}}$ & Features $^{\mathrm{c}}$ & $\begin{array}{c}\text { RA } \\
{[\mathrm{J} 2000]}\end{array}$ & $\begin{array}{c}\text { Dec } \\
{[\mathrm{J} 2000]}\end{array}$ & $z_{p h o t}$ & $\begin{array}{c}\mathrm{M}_{r}^{\prime \mathrm{d}} \\
{[\mathrm{mag}]}\end{array}$ & $\begin{array}{c}\mathrm{M}_{\star}^{\mathrm{e}} \\
{\left[\log \left(\mathrm{M}_{\odot}\right)\right]}\end{array}$ & $\begin{array}{c}\left(g^{\prime}-r^{\prime}\right)^{\mathrm{f}} \\
{[\mathrm{mag}]}\end{array}$ & Red/Blue ${ }^{\mathrm{g}}$ \\
\hline W1-191 & 0 & - & 33.662407 & -10.009939 & 0.150 & -23.08 & 11.28 & 0.573 & $\mathrm{R}$ \\
\hline W1-192 & 0 & - & 33.608509 & -9.889497 & 0.159 & -22.62 & 11.10 & 0.572 & $\mathrm{R}$ \\
\hline W1-195 & 0 & - & 33.598667 & -9.697360 & 0.095 & -21.88 & 10.77 & 0.543 & $\mathrm{R}$ \\
\hline W1-196 & 3 & $\mathrm{~L}$ & 33.847900 & -9.624586 & 0.183 & -22.97 & 11.25 & 0.586 & $\mathrm{R}$ \\
\hline W1-197 & 0 & - & 33.267975 & -9.594253 & 0.142 & -22.36 & 10.79 & 0.385 & $\mathrm{~B}$ \\
\hline W1-199 & 4 & $\mathrm{~A}, \mathrm{~L}$ & 33.399471 & -9.553778 & 0.118 & -22.03 & 10.83 & 0.546 & $\mathrm{R}$ \\
\hline W1-201 & 1 & - & 33.129509 & -9.486804 & 0.116 & -22.18 & 10.93 & 0.579 & $\mathrm{R}$ \\
\hline W1-202 & 4 & $\mathrm{~L}, \mathrm{~F}$ & 33.786945 & -9.493225 & 0.116 & -21.87 & 10.57 & 0.368 & $\mathrm{~B}$ \\
\hline W1-204 & 0 & - & 34.263840 & -10.192270 & 0.194 & -23.55 & 11.54 & 0.633 & $\mathrm{R}$ \\
\hline W1-205 & 0 & - & 34.649841 & -10.076246 & 0.117 & -22.10 & 10.68 & 0.382 & $\mathrm{~B}$ \\
\hline W1-206 & 3 & $\mathrm{~L}, \mathrm{H}$ & 34.827370 & -9.934042 & 0.136 & -21.97 & 10.30 & 0.091 & $\mathrm{~B}$ \\
\hline W1-207 & 2 & - & 34.744122 & -9.905999 & 0.113 & -21.79 & 10.70 & 0.511 & $\mathrm{~B}$ \\
\hline W1-208 & 4 & $\mathrm{H}$ & 34.780174 & -9.756317 & 0.090 & -21.35 & 10.59 & 0.574 & $\mathrm{R}$ \\
\hline W1-209 & 0 & - & 34.100971 & -9.735641 & 0.096 & -21.40 & 10.51 & 0.480 & B \\
\hline W1-210 & 1 & - & 34.885441 & -9.652877 & 0.106 & -21.98 & 10.46 & 0.231 & $\mathrm{~B}$ \\
\hline W1-211 & 0 & - & 34.813702 & -9.590844 & 0.082 & -21.03 & 10.11 & 0.251 & $\mathrm{~B}$ \\
\hline W1-214 & 1 & - & 35.832821 & -10.245669 & 0.100 & -21.68 & 10.76 & 0.600 & $\mathrm{R}$ \\
\hline W1-215 & 0 & - & 35.571705 & -10.056229 & 0.090 & -22.00 & 10.38 & 0.155 & $\mathrm{~B}$ \\
\hline W1-217 & 2 & - & 35.011841 & -9.981007 & 0.120 & -22.05 & 10.93 & 0.620 & $\mathrm{R}$ \\
\hline W1-218 & 2 & - & 35.271664 & -9.623956 & 0.101 & -21.62 & 10.48 & 0.381 & B \\
\hline W1-219 & 3 & $\mathrm{~F}$ & 35.197960 & -9.624628 & 0.088 & -21.23 & 10.50 & 0.532 & $\mathrm{R}$ \\
\hline W1-221 & 1 & - & 36.765987 & -10.246611 & 0.133 & -23.05 & 11.04 & 0.360 & $\mathrm{~B}$ \\
\hline W1-223 & 0 & - & 36.645374 & -10.239376 & 0.141 & -22.28 & 10.82 & 0.442 & $\mathrm{~B}$ \\
\hline W1-225 & 2 & - & 36.149441 & -9.866188 & 0.076 & -20.94 & 10.44 & 0.582 & $\mathrm{R}$ \\
\hline W1-227 & 0 & - & 36.250824 & -9.856068 & 0.082 & -21.14 & 10.40 & 0.479 & $\mathrm{~B}$ \\
\hline W1-229 & 2 & - & 36.340656 & -9.493792 & 0.084 & -21.31 & 10.23 & 0.261 & $\mathrm{~B}$ \\
\hline W1-231 & 4 & $\mathrm{~S}, \mathrm{M}$ & 36.662842 & -9.480710 & 0.120 & -22.19 & 10.98 & 0.614 & $\mathrm{R}$ \\
\hline W1-232 & 1 & - & 36.751221 & -9.476720 & 0.102 & -22.01 & 10.70 & 0.428 & B \\
\hline W1-234 & 2 & - & 36.893604 & -10.250633 & 0.120 & -21.96 & 10.93 & 0.654 & $\mathrm{R}$ \\
\hline W1-235 & 0 & - & 37.771584 & -10.183326 & 0.155 & -23.01 & 11.37 & 0.674 & $\mathrm{R}$ \\
\hline W1-236 & 4 & $\mathrm{~L}, \mathrm{M}$ & 37.049145 & -10.090625 & 0.140 & -22.65 & 11.04 & 0.503 & $\mathrm{~B}$ \\
\hline W1-237 & 1 & - & 37.142803 & -10.071088 & 0.086 & -21.02 & 10.42 & 0.538 & $\mathrm{R}$ \\
\hline W1-238 & 4 & $\mathrm{~A}, \mathrm{~S}, \mathrm{M}$ & 37.104496 & -10.032083 & 0.096 & -21.52 & 10.41 & 0.345 & $\mathrm{~B}$ \\
\hline W1-239 & 0 & - & 37.231674 & -10.012664 & 0.143 & -22.59 & 11.02 & 0.513 & $\mathrm{~B}$ \\
\hline W1-240 & 0 & - & 37.743439 & -9.927332 & 0.078 & -21.17 & 10.22 & 0.303 & $\mathrm{~B}$ \\
\hline W1-242 & 4 & M & 37.091965 & -9.887218 & 0.076 & -20.77 & 10.26 & 0.483 & $\mathrm{~B}$ \\
\hline W1-243 & 1 & - & 37.789600 & -9.833735 & 0.108 & -21.86 & 10.46 & 0.270 & B \\
\hline W1-244 & 1 & - & 37.106548 & -9.767576 & 0.176 & -23.28 & 11.21 & 0.431 & $\mathrm{~B}$ \\
\hline W1-245 & 2 & - & 37.630184 & -9.676272 & 0.094 & -21.51 & 10.31 & 0.260 & $\mathrm{~B}$ \\
\hline W1-246 & 1 & - & 36.920544 & -9.607326 & 0.115 & -21.88 & 10.75 & 0.524 & $\mathrm{R}$ \\
\hline W1-247 & 0 & - & 37.113533 & -9.492078 & 0.054 & -20.21 & 9.85 & 0.315 & $\mathrm{~B}$ \\
\hline W1-248 & 0 & - & 37.301956 & -9.508386 & 0.150 & -22.87 & 11.27 & 0.636 & $\mathrm{R}$ \\
\hline W1-249 & 3 & $\mathrm{~S}$ & 37.558922 & -9.441507 & 0.112 & -21.77 & 10.80 & 0.613 & $\mathrm{R}$ \\
\hline W1-250 & 0 & - & 37.161964 & -9.432535 & 0.099 & -21.42 & 10.63 & 0.587 & $\mathrm{R}$ \\
\hline
\end{tabular}


TABLE 4-Continued

\begin{tabular}{|c|c|c|c|c|c|c|c|c|c|}
\hline ID & Confidence $^{\mathrm{b}}$ & Features $^{\mathrm{c}}$ & $\begin{array}{c}\text { RA } \\
{[\mathrm{J} 2000]}\end{array}$ & $\begin{array}{c}\text { Dec } \\
{[\mathrm{J} 2000]}\end{array}$ & $z_{p h o t}$ & $\begin{array}{c}\mathrm{M}_{r}^{\prime \mathrm{d}} \\
{[\mathrm{mag}]}\end{array}$ & $\begin{array}{c}\mathrm{M}_{\star}^{\mathrm{e}} \\
{\left[\log \left(\mathrm{M}_{\odot}\right)\right]}\end{array}$ & $\begin{array}{c}\left(g^{\prime}-r^{\prime}\right)^{\mathrm{f}} \\
{[\mathrm{mag}]}\end{array}$ & Red/Blue ${ }^{\mathrm{g}}$ \\
\hline W1-255 & 1 & - & 37.872478 & -10.262243 & 0.180 & -23.02 & 11.31 & 0.619 & $\mathrm{R}$ \\
\hline W1-256 & 2 & - & 37.916256 & -10.148810 & 0.150 & -22.96 & 11.35 & 0.673 & $\mathrm{R}$ \\
\hline W1-257 & 0 & - & 38.147003 & -10.081855 & 0.121 & -22.81 & 11.23 & 0.618 & $\mathrm{R}$ \\
\hline W1-258 & 0 & - & 37.930779 & -10.007853 & 0.160 & -22.60 & 11.05 & 0.535 & $\mathrm{R}$ \\
\hline W1-259 & 0 & - & 38.407307 & -9.934512 & 0.194 & -23.37 & 11.32 & 0.501 & $\mathrm{~B}$ \\
\hline W1-260 & 2 & - & 37.911781 & -9.898181 & 0.152 & -22.97 & 11.35 & 0.672 & $\mathrm{R}$ \\
\hline W1-261 & 1 & - & 38.079853 & -9.817242 & 0.185 & -22.87 & 11.03 & 0.423 & $\mathrm{~B}$ \\
\hline W1-263 & 0 & - & 37.878296 & -9.718374 & 0.163 & -22.59 & 10.95 & 0.453 & $\mathrm{~B}$ \\
\hline W1-264 & 4 & $\mathrm{H}$ & 38.058838 & -9.531059 & 0.153 & -23.52 & 11.48 & 0.587 & $\mathrm{R}$ \\
\hline W1-265 & 1 & - & 38.535053 & -9.482280 & 0.166 & -22.63 & 11.06 & 0.529 & $\mathrm{~B}$ \\
\hline W1-266 & 4 & $\mathrm{~S}, \mathrm{~A}$ & 37.851940 & -9.358905 & 0.120 & -21.96 & 10.88 & 0.610 & $\mathrm{R}$ \\
\hline W1-267 & 0 & - & 30.322906 & -9.311458 & 0.141 & -22.44 & 10.83 & 0.397 & $\mathrm{~B}$ \\
\hline W1-270 & 0 & - & 31.020853 & -9.106462 & 0.090 & -21.53 & 10.67 & 0.578 & $\mathrm{R}$ \\
\hline W1-272 & 1 & - & 30.634579 & -8.937060 & 0.166 & -22.91 & 10.97 & 0.357 & B \\
\hline W1-273 & 4 & $\mathrm{~S}$ & 30.580271 & -8.897491 & 0.126 & -22.38 & 11.05 & 0.612 & $\mathrm{R}$ \\
\hline W1-274 & 3 & $\mathrm{~A}$ & 31.069664 & -8.868791 & 0.088 & -21.35 & 10.21 & 0.236 & $\mathrm{~B}$ \\
\hline W1-276 & 1 & - & 31.105413 & -8.840892 & 0.086 & -21.25 & 10.55 & 0.574 & $\mathrm{R}$ \\
\hline W1-277 & 2 & - & 30.353409 & -8.779748 & 0.065 & -20.61 & 10.36 & 0.626 & $\mathrm{R}$ \\
\hline W1-278 & 0 & - & 30.943834 & -8.557221 & 0.162 & -23.43 & 11.31 & 0.472 & $\mathrm{~B}$ \\
\hline W1-281 & 0 & - & 31.439518 & -9.213283 & 0.172 & -22.83 & 11.18 & 0.574 & $\mathrm{R}$ \\
\hline W1-283 & 2 & - & 31.273115 & -9.018538 & 0.067 & -20.45 & 10.12 & 0.469 & $\mathrm{~B}$ \\
\hline W1-284 & 0 & - & 31.317882 & -8.988440 & 0.158 & -22.69 & 11.18 & 0.622 & $\mathrm{R}$ \\
\hline W1-285 & 0 & - & 31.285994 & -8.912488 & 0.095 & -21.60 & 10.65 & 0.541 & $\mathrm{R}$ \\
\hline W1-288 & 4 & $\mathrm{~S}$ & 31.250198 & -8.710932 & 0.090 & -21.50 & 10.66 & 0.585 & $\mathrm{R}$ \\
\hline W1-289 & 0 & - & 31.652765 & -8.685903 & 0.121 & -22.12 & 10.91 & 0.579 & $\mathrm{R}$ \\
\hline W1-290 & 1 & - & 31.861036 & -8.613501 & 0.084 & -22.07 & 10.70 & 0.413 & $\mathrm{~B}$ \\
\hline W1-291 & 0 & - & 31.166386 & -8.608130 & 0.092 & -21.31 & 10.53 & 0.532 & $\mathrm{R}$ \\
\hline W1-293 & 1 & - & 32.003056 & -8.411625 & 0.146 & -23.05 & 11.19 & 0.498 & B \\
\hline W1-295 & 0 & - & 32.683708 & -9.228481 & 0.167 & -23.00 & 10.85 & 0.211 & $\mathrm{~B}$ \\
\hline W1-296 & 1 & - & 33.019218 & -9.128716 & 0.180 & -22.97 & 11.28 & 0.612 & $\mathrm{R}$ \\
\hline W1-297 & 4 & $\mathrm{~A}$ & 32.478092 & -9.082160 & 0.138 & -22.18 & 10.78 & 0.446 & $\mathrm{~B}$ \\
\hline W1-299 & 3 & $\mathrm{H}, \mathrm{L}$ & 33.058464 & -8.905539 & 0.148 & -22.35 & 11.03 & 0.603 & $\mathrm{R}$ \\
\hline W1-300 & 3 & $\mathrm{~A}$ & 32.199753 & -8.833365 & 0.150 & -22.77 & 11.18 & 0.596 & $\mathrm{R}$ \\
\hline W1-301 & 2 & - & 32.634121 & -8.820353 & 0.125 & -21.98 & 10.91 & 0.636 & $\mathrm{R}$ \\
\hline W1-302 & 1 & - & 32.197437 & -8.713874 & 0.060 & -20.52 & 10.27 & 0.578 & $\mathrm{R}$ \\
\hline W1-303 & 0 & - & 32.400856 & -8.496565 & 0.119 & -21.84 & 10.59 & 0.397 & $\mathrm{~B}$ \\
\hline W1-305 & 0 & - & 33.134842 & -9.216304 & 0.092 & -21.34 & 10.47 & 0.472 & B \\
\hline W1-306 & 2 & - & 33.321972 & -9.192824 & 0.087 & -21.07 & 10.23 & 0.353 & $\mathrm{~B}$ \\
\hline W1-308 & 0 & - & 33.592514 & -9.104666 & 0.155 & -23.01 & 11.26 & 0.577 & $\mathrm{R}$ \\
\hline W1-309 & 0 & - & 33.536820 & -9.013524 & 0.141 & -22.89 & 11.13 & 0.500 & $\mathrm{~B}$ \\
\hline W1-310 & 4 & M & 33.823746 & -9.017983 & 0.151 & -22.43 & 11.07 & 0.609 & $\mathrm{R}$ \\
\hline W1-311 & 2 & - & 33.430519 & -8.509462 & 0.175 & -22.83 & 10.70 & 0.137 & $\mathrm{~B}$ \\
\hline W1-313 & 1 & - & 34.078667 & -9.309242 & 0.192 & -23.04 & 11.18 & 0.495 & $\mathrm{~B}$ \\
\hline W1-314 & 3 & $\mathrm{~A}$ & 34.039547 & -9.233173 & 0.102 & -21.37 & 10.25 & 0.264 & $\mathrm{~B}$ \\
\hline
\end{tabular}


TABLE 4-Continued

\begin{tabular}{|c|c|c|c|c|c|c|c|c|c|}
\hline ID & Confidence $^{\mathrm{b}}$ & Features $^{\mathrm{c}}$ & $\begin{array}{c}\text { RA } \\
{[\mathrm{J} 2000]}\end{array}$ & $\begin{array}{c}\text { Dec } \\
{[\mathrm{J} 2000]}\end{array}$ & $z_{\text {phot }}$ & $\begin{array}{c}\mathrm{M}_{r}^{\prime \mathrm{d}} \\
{[\mathrm{mag}]}\end{array}$ & $\begin{array}{c}\mathrm{M}_{\star}^{\mathrm{e}} \\
{\left[\log \left(\mathrm{M}_{\odot}\right)\right]}\end{array}$ & $\begin{array}{c}\left(g^{\prime}-r^{\prime}\right)^{\mathrm{f}} \\
{[\mathrm{mag}]}\end{array}$ & Red/Blue ${ }^{\mathrm{g}}$ \\
\hline W1-315 & 3 & $\mathrm{~A}$ & 34.550709 & -9.030195 & 0.162 & -23.24 & 11.15 & 0.399 & B \\
\hline W1-316 & 4 & $\mathrm{~A}, \mathrm{~F}$ & 34.825420 & -9.022326 & 0.091 & -21.52 & 10.78 & 0.678 & $\mathrm{R}$ \\
\hline W1-317 & 2 & - & 34.049931 & -9.011267 & 0.070 & -21.21 & 10.39 & 0.446 & B \\
\hline W1-318 & 0 & - & 34.261826 & -8.989400 & 0.175 & -23.06 & 11.33 & 0.622 & $\mathrm{R}$ \\
\hline W1-319 & 0 & - & 34.405296 & -8.952990 & 0.118 & -22.44 & 11.12 & 0.657 & $\mathrm{R}$ \\
\hline W1-320 & 1 & - & 34.123463 & -8.897534 & 0.101 & -21.52 & 10.56 & 0.484 & $\mathrm{~B}$ \\
\hline W1-321 & 0 & - & 34.342415 & -8.880447 & 0.074 & -20.90 & 10.18 & 0.368 & B \\
\hline W1-322 & 1 & - & 34.492828 & -8.831283 & 0.153 & -22.62 & 10.91 & 0.409 & $\mathrm{~B}$ \\
\hline W1-323 & 4 & M & 34.588104 & -8.807344 & 0.064 & -20.36 & 10.04 & 0.438 & B \\
\hline W1-327 & 0 & - & 34.458447 & -8.667436 & 0.179 & -22.89 & 11.26 & 0.621 & $\mathrm{R}$ \\
\hline W1-331 & 2 & - & 34.875824 & -8.406717 & 0.181 & -23.82 & 11.40 & 0.411 & B \\
\hline W1-332 & 4 & - & 35.774872 & -9.247176 & 0.082 & -21.47 & 10.38 & 0.336 & B \\
\hline W1-333 & 1 & - & 35.354874 & -9.218025 & 0.138 & -22.21 & 10.66 & 0.324 & B \\
\hline W1-334 & 4 & $\mathrm{~A}$ & 35.370956 & -9.163622 & 0.082 & -21.30 & 10.48 & 0.488 & B \\
\hline W1-335 & 3 & $\mathrm{~A}$ & 35.399403 & -9.168033 & 0.154 & -22.73 & 11.00 & 0.449 & B \\
\hline W1-336 & 3 & $\mathrm{~A}, \mathrm{M}$ & 35.887535 & -9.034291 & 0.080 & -20.93 & 9.99 & 0.187 & B \\
\hline W1-337 & 0 & - & 35.589836 & -9.010030 & 0.067 & -20.81 & 10.32 & 0.518 & $\mathrm{R}$ \\
\hline W1-338 & 0 & - & 35.534065 & -8.869640 & 0.179 & -22.91 & 10.75 & 0.154 & B \\
\hline W1-339 & 4 & M & 35.867577 & -8.865355 & 0.180 & -23.40 & 11.50 & 0.653 & $\mathrm{R}$ \\
\hline W1-341 & 4 & $\mathrm{~F}$ & 35.079681 & -8.786392 & 0.120 & -22.37 & 11.07 & 0.639 & $\mathrm{R}$ \\
\hline W1-342 & 3 & $\mathrm{H}, \mathrm{L}$ & 35.289887 & -8.788397 & 0.146 & -22.46 & 11.09 & 0.617 & $\mathrm{R}$ \\
\hline W1-343 & 0 & - & 35.503918 & -8.693703 & 0.153 & -22.57 & 11.13 & 0.619 & $\mathrm{R}$ \\
\hline W1-344 & 0 & - & 35.171421 & -8.663119 & 0.150 & -22.99 & 11.31 & 0.632 & $\mathrm{R}$ \\
\hline W1-346 & 0 & - & 35.375603 & -8.637333 & 0.112 & -21.65 & 10.58 & 0.459 & B \\
\hline W1-347 & 2 & - & 35.080135 & -8.635124 & 0.100 & -21.97 & 10.84 & 0.575 & $\mathrm{R}$ \\
\hline W1-348 & 0 & - & 35.231770 & -8.594810 & 0.069 & -20.81 & 10.16 & 0.384 & B \\
\hline W1-349 & 0 & - & 35.464695 & -8.582577 & 0.154 & -22.73 & 11.15 & 0.580 & $\mathrm{R}$ \\
\hline W1-351 & 0 & - & 35.051662 & -8.539475 & 0.157 & -22.90 & 11.21 & 0.571 & $\mathrm{R}$ \\
\hline W1-352 & 0 & - & 35.480911 & -8.465271 & 0.120 & -22.17 & 10.96 & 0.607 & $\mathrm{R}$ \\
\hline W1-353 & 0 & - & 35.596168 & -8.425898 & 0.155 & -22.45 & 10.89 & 0.450 & B \\
\hline W1-354 & 0 & - & 36.714825 & -9.273974 & 0.092 & -21.89 & 10.59 & 0.380 & B \\
\hline W1-355 & 0 & - & 36.049198 & -9.177271 & 0.081 & -20.87 & 10.18 & 0.375 & B \\
\hline W1-356 & 0 & - & 36.843426 & -9.162097 & 0.149 & -22.58 & 11.02 & 0.521 & B \\
\hline W1-359 & 0 & - & 36.136131 & -9.097031 & 0.090 & -21.65 & 10.48 & 0.361 & B \\
\hline W1-360 & 4 & $\mathrm{~A}$ & 36.108280 & -9.102746 & 0.098 & -22.26 & 10.78 & 0.418 & B \\
\hline W1-361 & 1 & - & 36.086956 & -8.921722 & 0.112 & -21.71 & 10.55 & 0.407 & B \\
\hline W1-362 & 2 & - & 36.457710 & -8.800187 & 0.095 & -21.21 & 10.28 & 0.346 & B \\
\hline W1-363 & 4 & $\mathrm{H}, \mathrm{F}$ & 36.592510 & -8.789453 & 0.171 & -23.21 & 11.39 & 0.617 & $\mathrm{R}$ \\
\hline W1-364 & 2 & - & 36.242054 & -8.757931 & 0.154 & -22.49 & 11.10 & 0.618 & $\mathrm{R}$ \\
\hline W1-365 & 2 & - & 36.239533 & -8.745112 & 0.150 & -23.33 & 11.47 & 0.648 & $\mathrm{R}$ \\
\hline W1-366 & 0 & - & 36.359467 & -8.741807 & 0.120 & -22.22 & 11.00 & 0.623 & $\mathrm{R}$ \\
\hline W1-367 & 1 & - & 36.619774 & -8.724485 & 0.174 & -23.31 & 11.10 & 0.322 & B \\
\hline W1-368 & 0 & - & 36.337116 & -8.650527 & 0.108 & -21.85 & 10.50 & 0.312 & B \\
\hline W1-369 & 1 & - & 36.430889 & -8.623646 & 0.116 & -21.99 & 10.90 & 0.614 & $\mathrm{R}$ \\
\hline
\end{tabular}


TABLE 4-Continued

\begin{tabular}{|c|c|c|c|c|c|c|c|c|c|}
\hline ID & Confidence $^{\mathrm{b}}$ & Features $^{\mathrm{c}}$ & $\begin{array}{c}\text { RA } \\
{[\mathrm{J} 2000]}\end{array}$ & $\begin{array}{c}\text { Dec } \\
{[\mathrm{J} 2000]}\end{array}$ & $z_{\text {phot }}$ & $\begin{array}{c}\mathrm{M}_{r}^{\prime \mathrm{d}} \\
{[\mathrm{mag}]}\end{array}$ & $\begin{array}{c}\mathrm{M}_{\star}^{\mathrm{e}} \\
{\left[\log \left(\mathrm{M}_{\odot}\right)\right]}\end{array}$ & $\begin{array}{c}\left(g^{\prime}-r^{\prime}\right)^{\mathrm{f}} \\
{[\mathrm{mag}]}\end{array}$ & Red/Blue ${ }^{\mathrm{g}}$ \\
\hline W1-370 & 3 & M & 36.499699 & -8.604684 & 0.120 & -22.43 & 11.04 & 0.585 & $\mathrm{R}$ \\
\hline W1-373 & 0 & - & 36.081986 & -8.438305 & 0.060 & -21.01 & 10.54 & 0.646 & $\mathrm{R}$ \\
\hline W1-374 & 1 & - & 36.402958 & -8.427581 & 0.120 & -21.83 & 10.79 & 0.583 & $\mathrm{R}$ \\
\hline W1-375 & 4 & $\mathrm{~A}, \mathrm{M}$ & 36.299404 & -8.411882 & 0.132 & -22.09 & 10.90 & 0.589 & $\mathrm{R}$ \\
\hline W1-376 & 0 & - & 37.238090 & -9.177957 & 0.118 & -22.17 & 10.72 & 0.399 & B \\
\hline W1-377 & 2 & - & 37.480873 & -9.135452 & 0.092 & -21.29 & 10.50 & 0.515 & $\mathrm{~B}$ \\
\hline W1-379 & 2 & - & 37.785503 & -9.118298 & 0.150 & -22.94 & 11.25 & 0.591 & $\mathrm{R}$ \\
\hline W1-380 & 0 & - & 37.384773 & -9.020236 & 0.135 & -22.75 & 10.94 & 0.384 & $\mathrm{~B}$ \\
\hline W1-381 & 0 & - & 36.946880 & -8.946523 & 0.126 & -21.98 & 10.78 & 0.519 & B \\
\hline W1-383 & 0 & - & 37.022304 & -8.925438 & 0.151 & -22.86 & 11.27 & 0.635 & $\mathrm{R}$ \\
\hline W1-385 & 0 & - & 37.091084 & -8.802407 & 0.180 & -22.91 & 11.26 & 0.610 & $\mathrm{R}$ \\
\hline W1-386 & 4 & $\mathrm{~L}$ & 36.945919 & -8.625799 & 0.090 & -21.20 & 10.07 & 0.159 & B \\
\hline W1-387 & 0 & - & 37.325748 & -8.635001 & 0.175 & -22.92 & 11.23 & 0.586 & $\mathrm{R}$ \\
\hline W1-389 & 0 & - & 37.190685 & -8.570327 & 0.150 & -22.74 & 11.22 & 0.642 & $\mathrm{R}$ \\
\hline W1-390 & 4 & $\mathrm{~F}, \mathrm{M}$ & 37.642868 & -8.529206 & 0.120 & -22.72 & 11.25 & 0.669 & $\mathrm{R}$ \\
\hline W1-392 & 4 & $\mathrm{~L}, \mathrm{~F}$ & 37.189228 & -8.460222 & 0.060 & -20.68 & 9.74 & 0.053 & B \\
\hline W1-393 & 2 & - & 36.944374 & -8.400080 & 0.085 & -21.11 & 10.23 & 0.331 & $\mathrm{~B}$ \\
\hline W1-394 & 4 & $\mathrm{~F}, \mathrm{~A}$ & 38.354347 & -9.190852 & 0.120 & -22.60 & 11.18 & 0.648 & $\mathrm{R}$ \\
\hline W1-396 & 0 & - & 38.472805 & -9.042811 & 0.100 & -21.90 & 10.53 & 0.322 & B \\
\hline W1-397 & 0 & - & 38.289867 & -9.052702 & 0.150 & -22.74 & 11.21 & 0.630 & $\mathrm{R}$ \\
\hline W1-398 & 1 & - & 38.705605 & -8.957496 & 0.121 & -22.02 & 10.92 & 0.624 & $\mathrm{R}$ \\
\hline W1-399 & 0 & - & 38.137596 & -8.934146 & 0.180 & -23.24 & 11.41 & 0.625 & $\mathrm{R}$ \\
\hline W1-400 & 2 & - & 38.445270 & -8.846831 & 0.180 & -23.13 & 11.14 & 0.428 & B \\
\hline W1-402 & 0 & - & 38.619667 & -8.799247 & 0.079 & -21.25 & 10.33 & 0.376 & B \\
\hline W1-403 & 4 & $\mathrm{~S}$ & 38.007790 & -8.779521 & 0.127 & -22.39 & 11.06 & 0.624 & $\mathrm{R}$ \\
\hline W1-405 & 0 & - & 38.728970 & -8.710130 & 0.150 & -22.61 & 11.12 & 0.598 & $\mathrm{R}$ \\
\hline W1-409 & 0 & - & 30.508089 & -8.359650 & 0.169 & -23.48 & 11.45 & 0.576 & $\mathrm{R}$ \\
\hline W1-410 & 0 & - & 30.542717 & -8.197873 & 0.120 & -21.97 & 10.62 & 0.373 & B \\
\hline W1-412 & 0 & - & 30.976486 & -8.163961 & 0.185 & -23.70 & 11.39 & 0.445 & B \\
\hline W1-414 & 0 & - & 30.962435 & -8.090237 & 0.135 & -22.14 & 10.88 & 0.552 & $\mathrm{R}$ \\
\hline W1-415 & 0 & - & 31.009247 & -8.064521 & 0.150 & -23.40 & 11.48 & 0.631 & $\mathrm{R}$ \\
\hline W1-416 & 0 & - & 30.207500 & -8.041871 & 0.120 & -22.68 & 10.92 & 0.391 & B \\
\hline W1-418 & 0 & - & 30.425079 & -7.952306 & 0.121 & -22.18 & 10.98 & 0.628 & $\mathrm{R}$ \\
\hline W1-420 & 0 & - & 30.226286 & -7.910847 & 0.088 & -21.47 & 10.48 & 0.434 & B \\
\hline W1-421 & 0 & - & 31.143105 & -7.848433 & 0.187 & -23.52 & 11.35 & 0.477 & B \\
\hline W1-422 & 0 & - & 31.020010 & -7.836710 & 0.180 & -23.22 & 11.37 & 0.600 & $\mathrm{R}$ \\
\hline W1-423 & 1 & - & 31.069555 & -7.827113 & 0.138 & -22.65 & 10.96 & 0.437 & B \\
\hline W1-424 & 3 & $\mathrm{~L}$ & 30.243257 & -7.700147 & 0.182 & -23.29 & 11.41 & 0.614 & $\mathrm{R}$ \\
\hline W1-425 & 3 & $\mathrm{~S}$ & 30.555906 & -7.665324 & 0.071 & -20.79 & 10.08 & 0.312 & B \\
\hline W1-426 & 4 & M & 30.726990 & -7.650760 & 0.151 & -23.19 & 11.39 & 0.632 & $\mathrm{R}$ \\
\hline W1-427 & 0 & - & 30.198858 & -7.644201 & 0.150 & -22.52 & 11.10 & 0.606 & $\mathrm{R}$ \\
\hline W1-428 & 0 & - & 30.412691 & -7.636160 & 0.146 & -22.70 & 11.09 & 0.538 & $\mathrm{R}$ \\
\hline W1-431 & 3 & $\mathrm{~S}$ & 30.450819 & -7.590811 & 0.165 & -23.12 & 11.24 & 0.521 & B \\
\hline W1-432 & 0 & - & 30.435945 & -7.582360 & 0.090 & -21.15 & 10.23 & 0.322 & B \\
\hline
\end{tabular}


TABLE 4-Continued

\begin{tabular}{|c|c|c|c|c|c|c|c|c|c|}
\hline ID & Confidence $^{\mathrm{b}}$ & Features $^{\mathrm{c}}$ & $\begin{array}{c}\text { RA } \\
{[\mathrm{J} 2000]}\end{array}$ & $\begin{array}{c}\text { Dec } \\
{[\mathrm{J} 2000]}\end{array}$ & $z_{\text {phot }}$ & $\begin{array}{c}\mathrm{M}_{r}^{\prime \mathrm{d}} \\
{[\mathrm{mag}]}\end{array}$ & $\begin{array}{c}\mathrm{M}_{\star}^{\mathrm{e}} \\
{\left[\log \left(\mathrm{M}_{\odot}\right)\right]}\end{array}$ & $\begin{array}{c}\left(g^{\prime}-r^{\prime}\right)^{\mathrm{f}} \\
{[\mathrm{mag}]}\end{array}$ & Red/Blue ${ }^{\mathrm{g}}$ \\
\hline W1-433 & 0 & - & 30.346054 & -7.587179 & 0.152 & -22.73 & 11.19 & 0.614 & $\mathrm{R}$ \\
\hline W1-434 & 1 & - & 31.093634 & -7.585923 & 0.131 & -22.50 & 10.99 & 0.523 & B \\
\hline W1-435 & 0 & - & 30.941503 & -7.477212 & 0.090 & -21.48 & 10.68 & 0.601 & $\mathrm{R}$ \\
\hline W1-436 & 0 & - & 30.895050 & -7.469967 & 0.096 & -21.22 & 10.19 & 0.255 & B \\
\hline W1-438 & 3 & $\mathrm{H}$ & 31.237183 & -8.164458 & 0.095 & -21.39 & 10.74 & 0.693 & $\mathrm{R}$ \\
\hline W1-439 & 1 & - & 31.246838 & -8.137804 & 0.137 & -23.08 & 11.10 & 0.410 & $\mathrm{~B}$ \\
\hline W1-440 & 0 & - & 31.610189 & -8.036794 & 0.095 & -21.82 & 10.51 & 0.335 & B \\
\hline W1-441 & 4 & $\mathrm{~F}$ & 31.933512 & -8.009825 & 0.082 & -21.32 & 10.16 & 0.199 & $\mathrm{~B}$ \\
\hline W1-442 & 0 & - & 32.047100 & -7.983270 & 0.087 & -21.32 & 10.59 & 0.584 & $\mathrm{R}$ \\
\hline W1-443 & 0 & - & 32.001194 & -7.865070 & 0.111 & -22.41 & 10.76 & 0.345 & B \\
\hline W1-444 & 0 & - & 31.261265 & -7.851139 & 0.127 & -22.01 & 10.83 & 0.545 & $\mathrm{R}$ \\
\hline W1-446 & 0 & - & 31.933899 & -7.721002 & 0.150 & -22.48 & 11.08 & 0.608 & $\mathrm{R}$ \\
\hline W1-447 & 2 & - & 31.485830 & -7.679638 & 0.162 & -22.87 & 11.04 & 0.429 & B \\
\hline W1-448 & 0 & - & 32.059742 & -7.678372 & 0.110 & -22.36 & 10.81 & 0.405 & B \\
\hline W1-449 & 4 & $\mathrm{~S}$ & 31.629106 & -7.639644 & 0.151 & -23.06 & 11.31 & 0.600 & $\mathrm{R}$ \\
\hline W1-450 & 0 & - & 31.543543 & -7.594961 & 0.075 & -21.15 & 10.28 & 0.363 & B \\
\hline W1-451 & 0 & - & 31.715618 & -7.615479 & 0.181 & -23.14 & 11.36 & 0.622 & $\mathrm{R}$ \\
\hline W1-453 & 0 & - & 31.825010 & -7.469476 & 0.180 & -22.89 & 11.27 & 0.631 & $\mathrm{R}$ \\
\hline W1-454 & 0 & - & 32.117767 & -8.197608 & 0.107 & -21.78 & 10.62 & 0.443 & B \\
\hline W1-455 & 4 & $\mathrm{H}$ & 32.905582 & -8.173978 & 0.180 & -23.70 & 11.48 & 0.526 & B \\
\hline W1-458 & 0 & - & 32.158356 & -7.903811 & 0.187 & -23.13 & 11.03 & 0.326 & $\mathrm{~B}$ \\
\hline W1-459 & 0 & - & 32.264141 & -7.847484 & 0.180 & -23.45 & 11.23 & 0.387 & B \\
\hline W1-460 & 0 & - & 32.217712 & -7.846273 & 0.152 & -23.33 & 11.11 & 0.325 & B \\
\hline W1-461 & 0 & - & 32.575809 & -7.805628 & 0.168 & -23.41 & 11.45 & 0.605 & $\mathrm{R}$ \\
\hline W1-462 & 1 & - & 32.582008 & -7.725028 & 0.122 & -22.40 & 11.12 & 0.672 & $\mathrm{R}$ \\
\hline W1-463 & 0 & - & 32.723820 & -7.699318 & 0.169 & -23.08 & 11.28 & 0.573 & $\mathrm{R}$ \\
\hline W1-464 & 0 & - & 33.057972 & -7.583480 & 0.077 & -21.12 & 10.26 & 0.361 & B \\
\hline W1-467 & 1 & - & 32.582432 & -7.508636 & 0.076 & -20.87 & 10.11 & 0.313 & B \\
\hline W1-468 & 4 & $\mathrm{~A}, \mathrm{M}$ & 33.830673 & -8.359132 & 0.165 & -22.83 & 11.03 & 0.432 & B \\
\hline W1-469 & 2 & - & 33.867256 & -8.310109 & 0.176 & -23.10 & 11.22 & 0.507 & B \\
\hline W1-470 & 0 & - & 33.835342 & -8.175205 & 0.178 & -22.99 & 11.14 & 0.479 & B \\
\hline W1-472 & 2 & - & 33.874676 & -8.060620 & 0.144 & -23.05 & 11.31 & 0.603 & $\mathrm{R}$ \\
\hline W1-473 & 0 & - & 33.897575 & -8.065253 & 0.105 & -21.90 & 10.72 & 0.485 & B \\
\hline W1-474 & 0 & - & 33.429676 & -8.026393 & 0.150 & -22.97 & 11.36 & 0.681 & $\mathrm{R}$ \\
\hline W1-475 & 0 & - & 33.261536 & -7.775615 & 0.136 & -22.31 & 11.04 & 0.627 & $\mathrm{R}$ \\
\hline W1-478 & 2 & - & 34.021797 & -7.581259 & 0.180 & -22.88 & 11.12 & 0.502 & B \\
\hline W1-479 & 0 & - & 34.786030 & -8.375119 & 0.180 & -23.45 & 11.43 & 0.572 & $\mathrm{R}$ \\
\hline W1-481 & 0 & - & 34.747299 & -8.334416 & 0.158 & -22.53 & 10.94 & 0.463 & B \\
\hline W1-482 & 0 & - & 34.714664 & -8.320452 & 0.120 & -22.24 & 11.04 & 0.660 & $\mathrm{R}$ \\
\hline W1-483 & 0 & - & 34.557095 & -8.308818 & 0.177 & -22.75 & 11.00 & 0.435 & B \\
\hline W1-485 & 3 & M & 34.224121 & -8.198995 & 0.150 & -22.64 & 11.14 & 0.606 & $\mathrm{R}$ \\
\hline W1-487 & 0 & - & 34.392185 & -8.145350 & 0.110 & -21.75 & 10.57 & 0.412 & B \\
\hline W1-490 & 0 & - & 34.716053 & -8.061680 & 0.120 & -22.10 & 10.95 & 0.623 & $\mathrm{R}$ \\
\hline W1-491 & 0 & - & 34.160461 & -7.990540 & 0.180 & -22.94 & 11.28 & 0.618 & $\mathrm{R}$ \\
\hline
\end{tabular}


TABLE 4-Continued

\begin{tabular}{|c|c|c|c|c|c|c|c|c|c|}
\hline ID & Confidence $^{\mathrm{b}}$ & Features $^{c}$ & $\begin{array}{c}\text { RA } \\
{[\mathrm{J} 2000]}\end{array}$ & $\begin{array}{c}\text { Dec } \\
{[\mathrm{J} 2000]}\end{array}$ & $z_{\text {phot }}$ & $\begin{array}{c}\mathrm{M}_{r}^{\prime \mathrm{d}} \\
{[\mathrm{mag}]}\end{array}$ & $\begin{array}{c}\mathrm{M}_{\star}^{\mathrm{e}} \\
{\left[\log \left(\mathrm{M}_{\odot}\right)\right]}\end{array}$ & $\begin{array}{c}\left(g^{\prime}-r^{\prime}\right)^{\mathrm{f}} \\
{[\mathrm{mag}]}\end{array}$ & Red/Blue ${ }^{\mathrm{g}}$ \\
\hline W1-492 & 1 & - & 34.505474 & -7.911125 & 0.163 & -22.71 & 11.06 & 0.506 & B \\
\hline W1-493 & 2 & - & 34.827641 & -7.896359 & 0.179 & -23.33 & 11.24 & 0.446 & B \\
\hline W1-494 & 1 & - & 34.500744 & -7.898188 & 0.169 & -22.87 & 11.24 & 0.604 & $\mathrm{R}$ \\
\hline W1-497 & 0 & - & 34.864635 & -7.759414 & 0.120 & -22.19 & 11.01 & 0.646 & $\mathrm{R}$ \\
\hline W1-500 & 0 & - & 35.592838 & -8.326071 & 0.120 & -22.04 & 10.94 & 0.638 & $\mathrm{R}$ \\
\hline W1-501 & 2 & - & 35.382393 & -8.199127 & 0.117 & -21.78 & 10.73 & 0.545 & $\mathrm{R}$ \\
\hline W1-502 & 0 & - & 35.114681 & -8.187393 & 0.085 & -21.30 & 10.40 & 0.420 & B \\
\hline W1-503 & 4 & - & 35.469696 & -8.161862 & 0.101 & -21.41 & 10.24 & 0.236 & $\mathrm{~B}$ \\
\hline W1-504 & 0 & - & 35.128216 & -8.149623 & 0.176 & -23.03 & 10.87 & 0.220 & B \\
\hline W1-505 & 0 & - & 35.486008 & -7.971487 & 0.139 & -22.86 & 11.18 & 0.559 & $\mathrm{R}$ \\
\hline W1-507 & 1 & - & 35.278183 & -7.785260 & 0.080 & -20.78 & 10.03 & 0.272 & B \\
\hline W1-509 & 0 & - & 35.587955 & -7.683170 & 0.135 & -22.12 & 10.78 & 0.463 & B \\
\hline W1-510 & 0 & - & 36.668934 & -8.390828 & 0.110 & -21.88 & 10.69 & 0.468 & B \\
\hline W1-511 & 2 & - & 36.385536 & -8.346303 & 0.150 & -22.80 & 11.25 & 0.640 & $\mathrm{R}$ \\
\hline W1-512 & 4 & $\mathrm{H}, \mathrm{L}$ & 36.311661 & -8.347800 & 0.153 & -22.83 & 11.28 & 0.660 & $\mathrm{R}$ \\
\hline W1-513 & 0 & - & 36.433968 & -8.323864 & 0.098 & -21.42 & 10.41 & 0.387 & B \\
\hline W1-514 & 0 & - & 36.790924 & -8.305021 & 0.169 & -22.89 & 11.12 & 0.497 & $\mathrm{~B}$ \\
\hline W1-515 & 1 & - & 36.385372 & -8.268518 & 0.154 & -22.99 & 10.96 & 0.315 & B \\
\hline W1-516 & 0 & - & 36.587559 & -8.253731 & 0.147 & -22.62 & 11.14 & 0.607 & $\mathrm{R}$ \\
\hline W1-517 & 0 & - & 35.945682 & -8.213507 & 0.077 & -20.98 & 10.32 & 0.463 & B \\
\hline W1-518 & 2 & - & 36.389431 & -7.874524 & 0.090 & -21.42 & 10.32 & 0.306 & $\mathrm{~B}$ \\
\hline W1-524 & 2 & - & 36.028103 & -7.650845 & 0.135 & -22.26 & 10.99 & 0.605 & $\mathrm{R}$ \\
\hline W1-526 & 0 & - & 37.363289 & -8.256560 & 0.113 & -21.73 & 10.76 & 0.584 & $\mathrm{R}$ \\
\hline W1-527 & 0 & - & 37.558399 & -8.187303 & 0.150 & -22.56 & 11.14 & 0.630 & $\mathrm{R}$ \\
\hline W1-528 & 2 & - & 37.376488 & -8.128593 & 0.197 & -23.25 & 11.28 & 0.508 & B \\
\hline W1-531 & 1 & - & 37.778675 & -7.642822 & 0.106 & -21.51 & 10.40 & 0.339 & B \\
\hline W1-532 & 0 & - & 38.786820 & -8.373136 & 0.098 & -21.93 & 10.92 & 0.656 & $\mathrm{R}$ \\
\hline W1-533 & 0 & - & 37.854969 & -8.262341 & 0.164 & -22.67 & 10.97 & 0.438 & B \\
\hline W1-534 & 4 & $\mathrm{~F}$ & 37.892899 & -8.233998 & 0.090 & -21.79 & 10.90 & 0.697 & $\mathrm{R}$ \\
\hline W1-535 & 0 & - & 38.101021 & -8.194493 & 0.168 & -23.05 & 11.10 & 0.416 & B \\
\hline W1-536 & 4 & $\mathrm{~S}$ & 38.084538 & -8.188407 & 0.100 & -21.40 & 10.69 & 0.638 & $\mathrm{R}$ \\
\hline W1-537 & 1 & - & 38.599934 & -7.477653 & 0.181 & -22.77 & 10.74 & 0.196 & B \\
\hline W1-538 & 0 & - & 30.951706 & -7.426952 & 0.052 & -19.83 & 9.66 & 0.282 & B \\
\hline W1-539 & 0 & - & 30.726099 & -7.306275 & 0.179 & -23.23 & 11.43 & 0.654 & $\mathrm{R}$ \\
\hline W1-540 & 0 & - & 30.839809 & -7.277797 & 0.163 & -22.69 & 10.82 & 0.296 & B \\
\hline W1-541 & 0 & - & 30.487570 & -7.158827 & 0.180 & -22.89 & 11.30 & 0.652 & $\mathrm{R}$ \\
\hline W1-542 & 0 & - & 30.527094 & -7.132986 & 0.180 & -23.06 & 11.30 & 0.597 & $\mathrm{R}$ \\
\hline W1-543 & 0 & - & 30.565449 & -7.092520 & 0.188 & -23.05 & 11.03 & 0.357 & B \\
\hline W1-544 & 0 & - & 30.823832 & -7.060918 & 0.180 & -23.36 & 11.55 & 0.708 & $\mathrm{R}$ \\
\hline W1-545 & 0 & - & 30.927311 & -7.050244 & 0.147 & -22.40 & 10.84 & 0.418 & B \\
\hline W1-547 & 0 & - & 30.721937 & -6.979598 & 0.149 & -22.43 & 10.82 & 0.396 & B \\
\hline W1-548 & 0 & - & 30.198666 & -6.919785 & 0.180 & -23.24 & 11.42 & 0.641 & $\mathrm{R}$ \\
\hline W1-549 & 0 & - & 30.693359 & -6.907103 & 0.166 & -22.95 & 11.28 & 0.611 & $\mathrm{R}$ \\
\hline W1-550 & 4 & $\mathrm{~L}, \mathrm{~A}, \mathrm{M}$ & 30.536102 & -6.820979 & 0.120 & -22.69 & 11.24 & 0.669 & $\mathrm{R}$ \\
\hline
\end{tabular}


TABLE 4-Continued

\begin{tabular}{|c|c|c|c|c|c|c|c|c|c|}
\hline ID & Confidence $^{\mathrm{b}}$ & Features $^{c}$ & $\begin{array}{c}\text { RA } \\
{[\mathrm{J} 2000]}\end{array}$ & $\begin{array}{c}\text { Dec } \\
{[\mathrm{J} 2000]}\end{array}$ & $z_{\text {phot }}$ & $\begin{array}{c}\mathrm{M}_{r}^{\prime \mathrm{d}} \\
{[\mathrm{mag}]}\end{array}$ & $\begin{array}{c}\mathrm{M}_{\star}^{\mathrm{e}} \\
{\left[\log \left(\mathrm{M}_{\odot}\right)\right]}\end{array}$ & $\begin{array}{c}\left(g^{\prime}-r^{\prime}\right)^{\mathrm{f}} \\
{[\mathrm{mag}]}\end{array}$ & Red/Blue ${ }^{\mathrm{g}}$ \\
\hline W1-551 & 0 & - & 30.434628 & -6.787655 & 0.120 & -22.01 & 10.94 & 0.649 & $\mathrm{R}$ \\
\hline W1-552 & 0 & - & 31.019070 & -6.782743 & 0.090 & -21.17 & 10.62 & 0.660 & $\mathrm{R}$ \\
\hline W1-553 & 1 & - & 30.875198 & -6.755153 & 0.167 & -23.30 & 11.17 & 0.390 & B \\
\hline W1-554 & 3 & M & 30.566404 & -6.720750 & 0.121 & -22.44 & 11.13 & 0.660 & $\mathrm{R}$ \\
\hline W1-558 & 2 & - & 30.548346 & -6.627577 & 0.137 & -22.39 & 10.78 & 0.366 & B \\
\hline W1-560 & 1 & - & 30.670692 & -6.573194 & 0.170 & -23.34 & 11.19 & 0.400 & $\mathrm{~B}$ \\
\hline W1-561 & 3 & $\mathrm{~A}$ & 31.647377 & -7.404199 & 0.113 & -22.01 & 10.86 & 0.574 & $\mathrm{R}$ \\
\hline W1-564 & 0 & - & 31.719782 & -7.349049 & 0.084 & -21.04 & 10.40 & 0.514 & $\mathrm{R}$ \\
\hline W1-565 & 0 & - & 31.296377 & -7.214758 & 0.146 & -22.36 & 10.96 & 0.540 & $\mathrm{R}$ \\
\hline W1-567 & 0 & - & 31.978157 & -7.113281 & 0.107 & -22.61 & 10.83 & 0.332 & B \\
\hline W1-568 & 0 & - & 32.005310 & -7.043040 & 0.134 & -22.82 & 10.90 & 0.325 & B \\
\hline W1-569 & 0 & - & 31.201136 & -7.036559 & 0.150 & -22.78 & 11.22 & 0.622 & $\mathrm{R}$ \\
\hline W1-570 & 0 & - & 31.164372 & -6.910533 & 0.090 & -21.75 & 10.49 & 0.337 & B \\
\hline W1-571 & 2 & - & 31.958036 & -6.536741 & 0.063 & -20.39 & 10.16 & 0.527 & $\mathrm{R}$ \\
\hline W1-572 & 1 & - & 33.017510 & -7.378637 & 0.120 & -22.52 & 11.18 & 0.683 & $\mathrm{R}$ \\
\hline W1-573 & 0 & - & 32.791145 & -7.142229 & 0.151 & -22.54 & 11.10 & 0.604 & $\mathrm{R}$ \\
\hline W1-574 & 1 & - & 32.326901 & -7.121483 & 0.104 & -21.72 & 10.56 & 0.411 & $\mathrm{~B}$ \\
\hline W1-576 & 1 & - & 32.519753 & -6.981900 & 0.159 & -22.85 & 11.12 & 0.509 & B \\
\hline W1-577 & 0 & - & 32.561520 & -6.885199 & 0.091 & -21.70 & 10.53 & 0.389 & B \\
\hline W1-578 & 0 & - & 32.635807 & -6.873928 & 0.149 & -22.91 & 11.03 & 0.409 & B \\
\hline W1-579 & 2 & - & 32.144451 & -6.846555 & 0.180 & -23.18 & 11.40 & 0.640 & $\mathrm{R}$ \\
\hline W1-580 & 0 & - & 32.930675 & -6.826228 & 0.117 & -22.14 & 10.64 & 0.333 & B \\
\hline W1-581 & 0 & - & 32.131687 & -6.806475 & 0.120 & -22.23 & 10.99 & 0.612 & $\mathrm{R}$ \\
\hline W1-582 & 0 & - & 32.596760 & -6.755602 & 0.157 & -22.40 & 10.61 & 0.216 & B \\
\hline W1-584 & 0 & - & 32.631798 & -6.700701 & 0.137 & -22.19 & 10.97 & 0.616 & $\mathrm{R}$ \\
\hline W1-585 & 0 & - & 32.602192 & -6.688014 & 0.089 & -21.66 & 10.73 & 0.590 & $\mathrm{R}$ \\
\hline W1-587 & 4 & $\mathrm{~S}$ & 33.123718 & -7.464976 & 0.155 & -22.71 & 11.08 & 0.525 & B \\
\hline W1-588 & 4 & $\mathrm{~L}, \mathrm{H}$ & 33.593636 & -7.440462 & 0.150 & -22.61 & 11.13 & 0.604 & $\mathrm{R}$ \\
\hline W1-590 & 0 & - & 33.745148 & -7.243700 & 0.117 & -22.21 & 10.75 & 0.403 & B \\
\hline W1-592 & 3 & $\mathrm{~F}$ & 33.374947 & -7.184364 & 0.180 & -22.99 & 11.27 & 0.587 & $\mathrm{R}$ \\
\hline W1-593 & 0 & - & 33.654018 & -7.137939 & 0.068 & -21.00 & 10.21 & 0.354 & B \\
\hline W1-595 & 0 & - & 33.448841 & -7.130751 & 0.109 & -21.76 & 10.51 & 0.351 & B \\
\hline W1-596 & 0 & - & 33.940559 & -7.039578 & 0.150 & -22.59 & 11.11 & 0.592 & $\mathrm{R}$ \\
\hline W1-597 & 0 & - & 33.385891 & -7.030203 & 0.120 & -22.37 & 11.00 & 0.568 & $\mathrm{R}$ \\
\hline W1-598 & 0 & - & 33.798859 & -7.004424 & 0.123 & -22.36 & 10.75 & 0.355 & B \\
\hline W1-599 & 3 & M & 33.462406 & -6.951181 & 0.162 & -22.64 & 11.13 & 0.594 & $\mathrm{R}$ \\
\hline W1-600 & 0 & - & 33.340557 & -6.938809 & 0.114 & -21.97 & 10.82 & 0.555 & $\mathrm{R}$ \\
\hline W1-601 & 0 & - & 33.073338 & -6.879371 & 0.075 & -20.90 & 10.34 & 0.510 & B \\
\hline W1-602 & 2 & - & 33.282700 & -6.894778 & 0.098 & -21.47 & 10.64 & 0.573 & $\mathrm{R}$ \\
\hline W1-603 & 0 & - & 33.491337 & -6.793230 & 0.104 & -22.12 & 10.42 & 0.147 & B \\
\hline W1-604 & 1 & - & 33.372200 & -6.814934 & 0.150 & -22.82 & 11.19 & 0.585 & $\mathrm{R}$ \\
\hline W1-606 & 0 & - & 33.392811 & -6.781964 & 0.092 & -21.48 & 10.44 & 0.387 & B \\
\hline W1-609 & 0 & - & 34.578026 & -7.437544 & 0.120 & -22.35 & 10.79 & 0.395 & B \\
\hline W1-611 & 2 & - & 34.273319 & -7.278216 & 0.118 & -22.16 & 10.73 & 0.405 & B \\
\hline
\end{tabular}


TABLE 4-Continued

\begin{tabular}{|c|c|c|c|c|c|c|c|c|c|}
\hline ID & Confidence $^{\mathrm{b}}$ & Features $^{\mathrm{c}}$ & $\begin{array}{c}\text { RA } \\
{[\mathrm{J} 2000]}\end{array}$ & $\begin{array}{c}\text { Dec } \\
{[\mathrm{J} 2000]}\end{array}$ & $z_{\text {phot }}$ & $\begin{array}{c}\mathrm{M}_{r}^{\prime \mathrm{d}} \\
{[\mathrm{mag}]}\end{array}$ & $\begin{array}{c}\mathrm{M}_{\star}^{\mathrm{e}} \\
{\left[\log \left(\mathrm{M}_{\odot}\right)\right]}\end{array}$ & $\begin{array}{c}\left(g^{\prime}-r^{\prime}\right)^{\mathrm{f}} \\
{[\mathrm{mag}]}\end{array}$ & Red/Blue ${ }^{\mathrm{g}}$ \\
\hline W1-613 & 0 & - & 34.204697 & -7.262546 & 0.092 & -21.82 & 10.81 & 0.605 & $\mathrm{R}$ \\
\hline W1-614 & 0 & - & 34.539787 & -7.236530 & 0.154 & -22.66 & 10.91 & 0.387 & B \\
\hline W1-615 & 1 & - & 34.520210 & -7.215819 & 0.179 & -22.88 & 11.23 & 0.600 & $\mathrm{R}$ \\
\hline W1-616 & 0 & - & 34.192707 & -7.142098 & 0.071 & -20.60 & 9.89 & 0.217 & B \\
\hline W1-618 & 0 & - & 34.339634 & -6.897657 & 0.088 & -21.55 & 10.39 & 0.319 & B \\
\hline W1-619 & 0 & - & 34.684315 & -6.880465 & 0.185 & -23.62 & 11.33 & 0.426 & $\mathrm{~B}$ \\
\hline W1-620 & 0 & - & 34.459740 & -6.874788 & 0.121 & -21.96 & 10.85 & 0.586 & $\mathrm{R}$ \\
\hline W1-621 & 0 & - & 34.191956 & -6.859875 & 0.091 & -21.65 & 10.77 & 0.623 & $\mathrm{R}$ \\
\hline W1-622 & 3 & $\mathrm{~L}, \mathrm{~F}$ & 34.591911 & -6.840216 & 0.182 & -23.34 & 11.01 & 0.238 & B \\
\hline W1-623 & 0 & - & 34.308617 & -6.794756 & 0.131 & -22.72 & 11.05 & 0.492 & B \\
\hline W1-624 & 0 & - & 34.439461 & -6.784333 & 0.102 & -21.38 & 10.36 & 0.358 & B \\
\hline W1-626 & 3 & $\mathrm{~L}, \mathrm{~S}$ & 34.122917 & -6.758203 & 0.087 & -21.46 & 10.57 & 0.509 & B \\
\hline W1-627 & 0 & - & 34.071777 & -6.748288 & 0.173 & -23.83 & 11.60 & 0.584 & $\mathrm{R}$ \\
\hline W1-631 & 0 & - & 34.336746 & -6.637058 & 0.180 & -23.25 & 11.37 & 0.593 & $\mathrm{R}$ \\
\hline W1-635 & 0 & - & 34.146091 & -6.558790 & 0.091 & -21.48 & 10.26 & 0.226 & B \\
\hline W1-638 & 0 & - & 35.865505 & -7.236383 & 0.097 & -21.25 & 10.29 & 0.341 & B \\
\hline W1-639 & 4 & $\mathrm{~A}, \mathrm{M}$ & 35.580322 & -6.809200 & 0.150 & -22.82 & 11.23 & 0.614 & $\mathrm{R}$ \\
\hline W1-640 & 4 & $\mathrm{~A}, \mathrm{M}$ & 35.579639 & -6.810287 & 0.159 & -22.73 & 11.19 & 0.611 & $\mathrm{R}$ \\
\hline W1-642 & 1 & - & 35.639961 & -6.673075 & 0.130 & -22.54 & 10.62 & 0.174 & B \\
\hline W1-648 & 0 & - & 36.383587 & -7.247941 & 0.188 & -23.92 & 11.45 & 0.421 & B \\
\hline W1-649 & 2 & - & 35.967853 & -7.259577 & 0.180 & -23.32 & 11.43 & 0.617 & $\mathrm{R}$ \\
\hline W1-650 & 0 & - & 36.193508 & -7.256995 & 0.180 & -23.21 & 11.42 & 0.647 & $\mathrm{R}$ \\
\hline W1-651 & 0 & - & 35.963501 & -7.244499 & 0.190 & -23.11 & 11.37 & 0.641 & $\mathrm{R}$ \\
\hline W1-652 & 4 & $\mathrm{~F}, \mathrm{~S}$ & 36.492519 & -7.227057 & 0.171 & -22.91 & 11.21 & 0.567 & $\mathrm{R}$ \\
\hline W1-653 & 4 & $\mathrm{~L}, \mathrm{H}, \mathrm{F}$ & 36.547245 & -7.192420 & 0.147 & -22.49 & 11.12 & 0.638 & $\mathrm{R}$ \\
\hline W1-654 & 0 & - & 36.535072 & -7.164761 & 0.173 & -22.90 & 11.27 & 0.626 & $\mathrm{R}$ \\
\hline W1-655 & 4 & $\mathrm{~F}$ & 36.459648 & -7.048455 & 0.112 & -22.20 & 10.97 & 0.611 & $\mathrm{R}$ \\
\hline W1-657 & 1 & - & 36.442379 & -6.723511 & 0.179 & -22.94 & 11.30 & 0.634 & $\mathrm{R}$ \\
\hline W1-658 & 1 & - & 36.395885 & -6.642710 & 0.152 & -23.31 & 11.45 & 0.634 & $\mathrm{R}$ \\
\hline W1-660 & 0 & - & 36.008266 & -6.570480 & 0.126 & -22.63 & 10.80 & 0.298 & B \\
\hline W1-661 & 0 & - & 37.250568 & -7.322034 & 0.124 & -22.25 & 10.86 & 0.493 & B \\
\hline W1-663 & 4 & $\mathrm{~S}$ & 37.172844 & -7.030169 & 0.120 & -22.09 & 10.95 & 0.625 & $\mathrm{R}$ \\
\hline W1-664 & 0 & - & 37.598686 & -7.013355 & 0.074 & -21.30 & 10.25 & 0.282 & B \\
\hline W1-665 & 0 & - & 37.132874 & -6.875463 & 0.089 & -21.10 & 10.21 & 0.324 & B \\
\hline W1-666 & 3 & $\mathrm{~F}$ & 37.562542 & -6.875550 & 0.127 & -22.01 & 10.81 & 0.535 & $\mathrm{R}$ \\
\hline W1-669 & 0 & - & 38.650562 & -7.343497 & 0.090 & -21.43 & 10.36 & 0.337 & B \\
\hline W1-670 & 0 & - & 38.421764 & -7.233589 & 0.180 & -22.96 & 11.28 & 0.614 & $\mathrm{R}$ \\
\hline W1-671 & 4 & $\mathrm{~S}, \mathrm{~F}$ & 38.327782 & -6.914946 & 0.150 & -22.48 & 11.12 & 0.636 & $\mathrm{R}$ \\
\hline W1-672 & 0 & - & 38.645008 & -6.798974 & 0.180 & -22.95 & 11.27 & 0.606 & $\mathrm{R}$ \\
\hline W1-674 & 0 & - & 38.660545 & -6.718350 & 0.088 & -22.27 & 10.66 & 0.307 & B \\
\hline W1-676 & 3 & $\mathrm{~L}, \mathrm{~F}$ & 30.930340 & -6.481916 & 0.187 & -23.70 & 11.44 & 0.496 & B \\
\hline W1-677 & 4 & $\mathrm{~A}, \mathrm{M}$ & 30.938095 & -6.431514 & 0.090 & -21.46 & 10.69 & 0.619 & $\mathrm{R}$ \\
\hline W1-678 & 0 & - & 30.804665 & -6.387253 & 0.180 & -23.33 & 11.26 & 0.465 & B \\
\hline W1-680 & 0 & - & 30.822420 & -6.321378 & 0.118 & -22.22 & 10.59 & 0.260 & B \\
\hline
\end{tabular}


TABLE 4-Continued

\begin{tabular}{|c|c|c|c|c|c|c|c|c|c|}
\hline ID & Confidence $^{\mathrm{b}}$ & Features $^{\mathrm{c}}$ & $\begin{array}{c}\text { RA } \\
{[\mathrm{J} 2000]}\end{array}$ & $\begin{array}{c}\text { Dec } \\
{[\mathrm{J} 2000]}\end{array}$ & $z_{\text {phot }}$ & $\begin{array}{c}\mathrm{M}_{r}^{\prime \mathrm{d}} \\
{[\mathrm{mag}]}\end{array}$ & $\begin{array}{c}\mathrm{M}_{\star}^{\mathrm{e}} \\
{\left[\log \left(\mathrm{M}_{\odot}\right)\right]}\end{array}$ & $\begin{array}{c}\left(g^{\prime}-r^{\prime}\right)^{\mathrm{f}} \\
{[\mathrm{mag}]}\end{array}$ & Red/Blue ${ }^{\mathrm{g}}$ \\
\hline W1-681 & 0 & - & 30.443417 & -6.287400 & 0.067 & -21.22 & 10.61 & 0.635 & $\mathrm{R}$ \\
\hline W1-683 & 4 & $\mathrm{~A}$ & 30.218664 & -6.293143 & 0.150 & -22.66 & 11.19 & 0.637 & $\mathrm{R}$ \\
\hline W1-684 & 2 & - & 30.203859 & -6.285913 & 0.162 & -22.83 & 11.27 & 0.646 & $\mathrm{R}$ \\
\hline W1-685 & 0 & - & 30.837103 & -6.284542 & 0.179 & -23.34 & 11.35 & 0.537 & B \\
\hline W1-687 & 0 & - & 30.619959 & -6.157635 & 0.156 & -23.02 & 11.08 & 0.414 & B \\
\hline W1-688 & 0 & - & 30.285782 & -6.134123 & 0.123 & -22.17 & 10.99 & 0.637 & $\mathrm{R}$ \\
\hline W1-689 & 0 & - & 30.946752 & -6.040495 & 0.120 & -22.02 & 10.94 & 0.646 & $\mathrm{R}$ \\
\hline W1-690 & 2 & - & 30.864016 & -6.024021 & 0.062 & -20.48 & 10.30 & 0.622 & $\mathrm{R}$ \\
\hline W1-691 & 2 & - & 30.933336 & -5.997407 & 0.150 & -22.67 & 11.19 & 0.640 & $\mathrm{R}$ \\
\hline W1-692 & 0 & - & 31.042820 & -5.819750 & 0.134 & -22.18 & 10.68 & 0.352 & B \\
\hline W1-694 & 0 & - & 30.705420 & -5.733137 & 0.150 & -22.41 & 11.07 & 0.619 & $\mathrm{R}$ \\
\hline W1-700 & 0 & - & 32.061672 & -6.316863 & 0.165 & -22.71 & 11.00 & 0.447 & B \\
\hline W1-701 & 0 & - & 31.437141 & -6.286194 & 0.096 & -21.61 & 10.65 & 0.528 & $\mathrm{R}$ \\
\hline W1-702 & 0 & - & 31.413164 & -6.256960 & 0.111 & -21.81 & 10.56 & 0.378 & B \\
\hline W1-703 & 4 & $\mathrm{~S}$ & 31.229925 & -6.248841 & 0.099 & -21.54 & 10.70 & 0.600 & $\mathrm{R}$ \\
\hline W1-704 & 3 & M & 31.439827 & -6.230363 & 0.097 & -21.39 & 10.56 & 0.529 & $\mathrm{R}$ \\
\hline W1-705 & 0 & - & 31.248655 & -6.199285 & 0.180 & -22.88 & 11.26 & 0.626 & $\mathrm{R}$ \\
\hline W1-706 & 4 & $\mathrm{~S}$ & 31.433109 & -6.161631 & 0.104 & -21.51 & 10.42 & 0.361 & B \\
\hline W1-708 & 0 & - & 31.438442 & -6.154890 & 0.093 & -21.42 & 10.57 & 0.531 & $\mathrm{R}$ \\
\hline W1-709 & 0 & - & 31.511915 & -6.096455 & 0.145 & -22.67 & 10.89 & 0.368 & B \\
\hline W1-710 & 4 & $\mathrm{~A}$ & 31.520147 & -6.054352 & 0.091 & -22.10 & 10.92 & 0.595 & $\mathrm{R}$ \\
\hline W1-711 & 0 & - & 31.974148 & -6.000361 & 0.150 & -22.40 & 10.86 & 0.437 & B \\
\hline W1-713 & 4 & $\mathrm{~A}, \mathrm{~S}$ & 31.985376 & -5.700945 & 0.129 & -22.02 & 10.88 & 0.586 & $\mathrm{R}$ \\
\hline W1-715 & 0 & - & 32.891781 & -6.503044 & 0.093 & -21.37 & 10.35 & 0.347 & B \\
\hline W1-718 & 0 & - & 32.933716 & -6.383487 & 0.173 & -22.92 & 11.22 & 0.568 & $\mathrm{R}$ \\
\hline W1-719 & 0 & - & 32.468521 & -6.368835 & 0.060 & -20.35 & 9.73 & 0.158 & B \\
\hline W1-720 & 1 & - & 32.424068 & -6.319464 & 0.116 & -22.08 & 10.61 & 0.331 & B \\
\hline W1-721 & 0 & - & 32.788055 & -6.306238 & 0.144 & -23.32 & 11.35 & 0.547 & $\mathrm{R}$ \\
\hline W1-722 & 3 & $\mathrm{~S}$ & 32.266251 & -6.195110 & 0.150 & -23.21 & 11.38 & 0.614 & $\mathrm{R}$ \\
\hline W1-723 & 0 & - & 32.171623 & -6.199658 & 0.117 & -21.98 & 10.57 & 0.330 & B \\
\hline W1-724 & 0 & - & 32.484177 & -6.154427 & 0.127 & -22.40 & 10.98 & 0.549 & $\mathrm{R}$ \\
\hline W1-725 & 1 & - & 32.614037 & -5.983314 & 0.056 & -20.21 & 9.88 & 0.345 & B \\
\hline W1-726 & 1 & - & 32.138538 & -5.927599 & 0.150 & -22.67 & 11.13 & 0.584 & $\mathrm{R}$ \\
\hline W1-727 & 0 & - & 32.142296 & -5.820430 & 0.063 & -20.31 & 10.21 & 0.608 & $\mathrm{R}$ \\
\hline W1-728 & 0 & - & 32.291225 & -5.791084 & 0.134 & -22.90 & 11.02 & 0.402 & B \\
\hline W1-730 & 0 & - & 32.198467 & -5.787222 & 0.082 & -21.65 & 10.46 & 0.352 & B \\
\hline W1-731 & 0 & - & 32.237541 & -5.699671 & 0.156 & -22.64 & 11.09 & 0.555 & $\mathrm{R}$ \\
\hline W1-733 & 0 & - & 32.895180 & -5.601454 & 0.102 & -21.64 & 10.38 & 0.282 & B \\
\hline W1-734 & 0 & - & 33.082310 & -6.448335 & 0.060 & -20.19 & 10.16 & 0.605 & $\mathrm{R}$ \\
\hline W1-735 & 0 & - & 33.763390 & -6.446371 & 0.082 & -21.39 & 10.56 & 0.528 & $\mathrm{R}$ \\
\hline W1-736 & 0 & - & 33.412563 & -6.444153 & 0.120 & -22.09 & 10.95 & 0.626 & $\mathrm{R}$ \\
\hline W1-739 & 0 & - & 33.369022 & -6.370758 & 0.161 & -22.54 & 10.93 & 0.450 & B \\
\hline W1-741 & 1 & - & 33.071938 & -6.155965 & 0.157 & -22.63 & 10.71 & 0.226 & B \\
\hline W1-742 & 0 & - & 33.628422 & -6.136790 & 0.167 & -23.33 & 11.23 & 0.433 & B \\
\hline
\end{tabular}


TABLE 4-Continued

\begin{tabular}{|c|c|c|c|c|c|c|c|c|c|}
\hline ID & Confidence $^{\mathrm{b}}$ & Features $^{\mathrm{c}}$ & $\begin{array}{c}\text { RA } \\
{[\mathrm{J} 2000]}\end{array}$ & $\begin{array}{c}\text { Dec } \\
{[\mathrm{J} 2000]}\end{array}$ & $z_{\text {phot }}$ & $\begin{array}{c}\mathrm{M}_{r}^{\prime \mathrm{d}} \\
{[\mathrm{mag}]}\end{array}$ & $\begin{array}{c}\mathrm{M}_{\star}^{\mathrm{e}} \\
{\left[\log \left(\mathrm{M}_{\odot}\right)\right]}\end{array}$ & $\begin{array}{c}\left(g^{\prime}-r^{\prime}\right)^{\mathrm{f}} \\
{[\mathrm{mag}]}\end{array}$ & Red/Blue ${ }^{\mathrm{g}}$ \\
\hline W1-743 & 0 & - & 33.227508 & -6.136630 & 0.193 & -23.13 & 11.35 & 0.613 & $\mathrm{R}$ \\
\hline W1-744 & 2 & - & 33.567810 & -6.099414 & 0.152 & -22.97 & 11.28 & 0.609 & $\mathrm{R}$ \\
\hline W1-745 & 0 & - & 33.491737 & -5.965949 & 0.163 & -23.35 & 11.10 & 0.308 & B \\
\hline W1-746 & 0 & - & 33.171925 & -5.818039 & 0.125 & -22.07 & 10.69 & 0.401 & B \\
\hline W1-747 & 1 & - & 33.108700 & -5.812184 & 0.195 & -23.95 & 11.38 & 0.343 & B \\
\hline W1-749 & 0 & - & 34.816997 & -6.439240 & 0.106 & -21.67 & 10.38 & 0.269 & $\mathrm{~B}$ \\
\hline W1-751 & 0 & - & 34.724300 & -6.280736 & 0.076 & -21.33 & 10.39 & 0.396 & B \\
\hline W1-753 & 4 & - & 34.895359 & -6.105222 & 0.101 & -22.01 & 10.76 & 0.484 & $\mathrm{~B}$ \\
\hline W1-754 & 0 & - & 34.803513 & -6.038069 & 0.093 & -21.51 & 10.57 & 0.500 & B \\
\hline W1-755 & 0 & - & 34.817082 & -6.054928 & 0.099 & -21.61 & 10.64 & 0.524 & $\mathrm{R}$ \\
\hline W1-758 & 0 & - & 34.140095 & -6.041120 & 0.087 & -21.45 & 10.30 & 0.275 & B \\
\hline W1-760 & 2 & - & 34.787804 & -5.910472 & 0.151 & -22.66 & 11.18 & 0.634 & $\mathrm{R}$ \\
\hline W1-761 & 4 & $\mathrm{~L}, \mathrm{H}$ & 34.852253 & -5.904421 & 0.149 & -22.47 & 11.08 & 0.609 & $\mathrm{R}$ \\
\hline W1-762 & 0 & - & 34.794674 & -5.844139 & 0.140 & -22.38 & 10.88 & 0.465 & B \\
\hline W1-763 & 0 & - & 35.603085 & -6.461341 & 0.052 & -19.90 & 9.92 & 0.494 & B \\
\hline W1-764 & 2 & - & 35.139710 & -6.434043 & 0.178 & -22.84 & 11.04 & 0.445 & B \\
\hline W1-765 & 2 & - & 35.602489 & -6.267052 & 0.066 & -20.55 & 10.29 & 0.589 & $\mathrm{R}$ \\
\hline W1-766 & 4 & M & 35.749302 & -6.188344 & 0.121 & -22.18 & 10.97 & 0.614 & $\mathrm{R}$ \\
\hline W1-768 & 0 & - & 35.561119 & -6.142495 & 0.149 & -22.44 & 10.70 & 0.278 & B \\
\hline W1-769 & 0 & - & 35.364758 & -5.823608 & 0.071 & -21.51 & 10.40 & 0.343 & B \\
\hline W1-770 & 0 & - & 35.038280 & -5.710916 & 0.091 & -21.85 & 10.51 & 0.321 & $\mathrm{~B}$ \\
\hline W1-773 & 1 & - & 36.102467 & -6.473917 & 0.180 & -23.06 & 11.33 & 0.623 & $\mathrm{R}$ \\
\hline W1-774 & 0 & - & 36.097210 & -6.412980 & 0.090 & -21.27 & 10.57 & 0.579 & $\mathrm{R}$ \\
\hline W1-776 & 0 & - & 36.268723 & -6.310822 & 0.150 & -22.52 & 10.87 & 0.401 & B \\
\hline W1-777 & 0 & - & 36.087307 & -6.152583 & 0.120 & -22.66 & 11.12 & 0.575 & $\mathrm{R}$ \\
\hline W1-778 & 4 & $\mathrm{~L}, \mathrm{~F}, \mathrm{M}$ & 36.626690 & -6.157453 & 0.193 & -23.34 & 10.90 & 0.139 & B \\
\hline W1-779 & 0 & - & 36.178097 & -6.140841 & 0.101 & -22.06 & 10.73 & 0.441 & B \\
\hline W1-780 & 2 & - & 36.373215 & -6.102281 & 0.163 & -23.19 & 11.16 & 0.420 & B \\
\hline W1-781 & 0 & - & 36.289196 & -6.073741 & 0.149 & -22.76 & 11.16 & 0.576 & $\mathrm{R}$ \\
\hline W1-782 & 0 & - & 36.576565 & -6.092054 & 0.062 & -20.42 & 10.25 & 0.602 & $\mathrm{R}$ \\
\hline W1-783 & 0 & - & 36.365509 & -6.044162 & 0.150 & -22.60 & 11.05 & 0.536 & $\mathrm{R}$ \\
\hline W1-785 & 0 & - & 36.127052 & -5.918778 & 0.072 & -21.29 & 10.30 & 0.331 & B \\
\hline W1-786 & 0 & - & 36.289810 & -5.939649 & 0.124 & -21.96 & 10.85 & 0.583 & $\mathrm{R}$ \\
\hline W1-787 & 0 & - & 36.308022 & -5.911330 & 0.060 & -20.46 & 10.26 & 0.596 & $\mathrm{R}$ \\
\hline W1-788 & 0 & - & 36.403004 & -5.873018 & 0.072 & -20.72 & 10.16 & 0.411 & B \\
\hline W1-790 & 0 & - & 36.444675 & -5.742699 & 0.060 & -20.20 & 10.15 & 0.590 & $\mathrm{R}$ \\
\hline W1-793 & 0 & - & 36.961597 & -6.403534 & 0.094 & -21.34 & 10.24 & 0.260 & B \\
\hline W1-794 & 0 & - & 37.707981 & -6.362548 & 0.128 & -22.00 & 10.79 & 0.512 & B \\
\hline W1-795 & 0 & - & 37.149994 & -5.940963 & 0.099 & -21.71 & 10.40 & 0.270 & B \\
\hline W1-796 & 0 & - & 37.332607 & -5.939698 & 0.093 & -21.23 & 10.17 & 0.241 & B \\
\hline W1-798 & 0 & - & 37.225613 & -5.622992 & 0.082 & -20.97 & 10.36 & 0.503 & B \\
\hline W1-800 & 3 & $\mathrm{~F}$ & 38.652245 & -6.375801 & 0.150 & -23.17 & 11.33 & 0.586 & $\mathrm{R}$ \\
\hline W1-802 & 0 & - & 38.540077 & -6.128997 & 0.072 & -20.87 & 10.04 & 0.251 & B \\
\hline W1-804 & 0 & - & 38.631935 & -6.076873 & 0.163 & -22.78 & 10.66 & 0.123 & B \\
\hline
\end{tabular}


TABLE 4-Continued

\begin{tabular}{|c|c|c|c|c|c|c|c|c|c|}
\hline ID & Confidence $^{\mathrm{b}}$ & Features $^{\mathrm{c}}$ & $\begin{array}{c}\text { RA } \\
{[\mathrm{J} 2000]}\end{array}$ & $\begin{array}{c}\text { Dec } \\
{[\mathrm{J} 2000]}\end{array}$ & $z_{p h o t}$ & $\begin{array}{c}\mathrm{M}_{r}^{\prime \mathrm{d}} \\
{[\mathrm{mag}]}\end{array}$ & $\begin{array}{c}\mathrm{M}_{\star}^{\mathrm{e}} \\
{\left[\log \left(\mathrm{M}_{\odot}\right)\right]}\end{array}$ & $\begin{array}{c}\left(g^{\prime}-r^{\prime}\right)^{\mathrm{f}} \\
{[\mathrm{mag}]}\end{array}$ & Red/Blue ${ }^{\mathrm{g}}$ \\
\hline W1-806 & 1 & - & 38.690754 & -5.711457 & 0.180 & -23.55 & 11.50 & 0.596 & $\mathrm{R}$ \\
\hline W2-1 & 0 & - & 132.259323 & -5.652067 & 0.090 & -21.64 & 10.51 & 0.397 & $\mathrm{~B}$ \\
\hline $\mathrm{W} 2-2$ & 1 & - & 132.776993 & -5.645323 & 0.119 & -22.67 & 11.16 & 0.606 & $\mathrm{R}$ \\
\hline $\mathrm{W} 2-6$ & 0 & - & 132.715134 & -5.517411 & 0.092 & -21.34 & 10.63 & 0.610 & $\mathrm{R}$ \\
\hline W2-9 & 0 & - & 132.413864 & -5.281756 & 0.108 & -21.94 & 10.87 & 0.609 & $\mathrm{R}$ \\
\hline W2-12 & 4 & $\mathrm{~F}$ & 132.406158 & -5.236991 & 0.095 & -21.50 & 10.50 & 0.438 & $\mathrm{~B}$ \\
\hline W2-13 & 0 & - & 132.334045 & -5.225745 & 0.142 & -22.28 & 11.02 & 0.618 & $\mathrm{R}$ \\
\hline W2-15 & 0 & - & 132.288940 & -5.148350 & 0.139 & -22.20 & 10.63 & 0.306 & $\mathrm{~B}$ \\
\hline W2-18 & 1 & - & 132.794662 & -5.089084 & 0.130 & -22.24 & 10.91 & 0.539 & $\mathrm{R}$ \\
\hline W2-19 & 1 & - & 132.672485 & -5.028939 & 0.091 & -21.63 & 10.72 & 0.586 & $\mathrm{R}$ \\
\hline $\mathrm{W} 2-20$ & 0 & - & 132.753586 & -5.024798 & 0.093 & -21.37 & 10.56 & 0.539 & $\mathrm{R}$ \\
\hline W2-22 & 0 & - & 132.648727 & -4.999977 & 0.041 & -19.30 & 9.40 & 0.243 & $\mathrm{~B}$ \\
\hline W2-24 & 0 & - & 132.658295 & -5.003128 & 0.070 & -20.81 & 10.40 & 0.597 & $\mathrm{R}$ \\
\hline $\mathrm{W} 2-27$ & 0 & - & 132.607681 & -4.976483 & 0.050 & -19.77 & 9.98 & 0.589 & $\mathrm{R}$ \\
\hline W2-29 & 0 & - & 132.390610 & -4.935987 & 0.150 & -23.23 & 11.41 & 0.635 & $\mathrm{R}$ \\
\hline $\mathrm{W} 2-30$ & 0 & - & 132.363586 & -4.918815 & 0.121 & -22.38 & 11.05 & 0.611 & $\mathrm{R}$ \\
\hline W2-33 & 0 & - & 132.521866 & -4.860580 & 0.091 & -21.68 & 10.79 & 0.626 & $\mathrm{R}$ \\
\hline W2-39 & 1 & - & 133.931671 & -5.551276 & 0.124 & -22.10 & 10.93 & 0.604 & $\mathrm{R}$ \\
\hline W2-42 & 4 & $\mathrm{~S}$ & 133.883636 & -5.512226 & 0.130 & -22.60 & 10.95 & 0.448 & $\mathrm{~B}$ \\
\hline $\mathrm{W} 2-43$ & 0 & - & 133.842102 & -5.473709 & 0.139 & -22.28 & 10.87 & 0.491 & $\mathrm{~B}$ \\
\hline $\mathrm{W} 2-47$ & 0 & - & 133.259811 & -5.379779 & 0.067 & -20.45 & 10.16 & 0.507 & $\mathrm{R}$ \\
\hline W2-49 & 1 & - & 133.513626 & -5.344761 & 0.102 & -21.59 & 10.44 & 0.347 & $\mathrm{~B}$ \\
\hline $\mathrm{W} 2-50$ & 0 & - & 133.386047 & -5.337523 & 0.055 & -20.22 & 10.17 & 0.598 & $\mathrm{R}$ \\
\hline W2-51 & 0 & - & 133.501862 & -5.217670 & 0.121 & -22.06 & 10.46 & 0.201 & $\mathrm{~B}$ \\
\hline W2-52 & 0 & - & 133.459320 & -5.208953 & 0.096 & -21.45 & 10.52 & 0.469 & $\mathrm{~B}$ \\
\hline $\mathrm{W} 2-55$ & 1 & - & 133.132980 & -5.110237 & 0.166 & -22.67 & 10.73 & 0.223 & $\mathrm{~B}$ \\
\hline W2-56 & 0 & - & 133.229568 & -5.059359 & 0.062 & -20.41 & 10.25 & 0.599 & $\mathrm{R}$ \\
\hline W2-57 & 0 & - & 133.200607 & -5.044389 & 0.075 & -20.70 & 10.34 & 0.582 & $\mathrm{R}$ \\
\hline W2-59 & 0 & - & 133.355881 & -4.995103 & 0.144 & -22.27 & 10.88 & 0.506 & $\mathrm{~B}$ \\
\hline $\mathrm{W} 2-60$ & 0 & - & 133.087677 & -4.951773 & 0.158 & -23.13 & 11.19 & 0.473 & $\mathrm{~B}$ \\
\hline W2-67 & 0 & - & 134.359970 & -5.661165 & 0.152 & -22.66 & 11.17 & 0.624 & $\mathrm{R}$ \\
\hline W2-68 & 4 & $\mathrm{~A}$ & 134.097809 & -5.648972 & 0.092 & -21.53 & 10.74 & 0.644 & $\mathrm{R}$ \\
\hline W2-69 & 4 & $\mathrm{~A}$ & 134.096191 & -5.646927 & 0.090 & -21.84 & 10.90 & 0.669 & $\mathrm{R}$ \\
\hline $\mathrm{W} 2-70$ & 2 & - & 134.447235 & -5.631858 & 0.173 & -23.66 & 11.34 & 0.419 & $\mathrm{~B}$ \\
\hline $\mathrm{W} 2-74$ & 0 & - & 134.630676 & -5.496285 & 0.097 & -21.49 & 10.55 & 0.491 & $\mathrm{~B}$ \\
\hline $\mathrm{W} 2-76$ & 0 & - & 134.025543 & -5.465482 & 0.100 & -21.40 & 10.63 & 0.588 & $\mathrm{R}$ \\
\hline $\mathrm{W} 2-77$ & 4 & M & 134.633362 & -5.460415 & 0.120 & -22.17 & 10.99 & 0.634 & $\mathrm{R}$ \\
\hline W2-81 & 0 & - & 134.870483 & -5.411745 & 0.144 & -22.69 & 11.12 & 0.563 & $\mathrm{R}$ \\
\hline W2-82 & 0 & - & 134.835327 & -5.409752 & 0.142 & -22.77 & 11.01 & 0.436 & $\mathrm{~B}$ \\
\hline W2-83 & 0 & - & 134.719360 & -5.413341 & 0.120 & -22.15 & 10.96 & 0.615 & $\mathrm{R}$ \\
\hline W2-84 & 1 & - & 134.339188 & -5.404944 & 0.114 & -21.71 & 10.78 & 0.612 & $\mathrm{R}$ \\
\hline W2-87 & 1 & - & 134.072891 & -5.385250 & 0.087 & -21.44 & 10.47 & 0.429 & B \\
\hline W2-88 & 4 & $\mathrm{~A}$ & 134.738327 & -5.350936 & 0.117 & -22.71 & 11.19 & 0.621 & $\mathrm{R}$ \\
\hline W2-89 & 3 & $\mathrm{~L}, \mathrm{~A}$ & 134.826599 & -5.350978 & 0.120 & -22.06 & 10.91 & 0.608 & $\mathrm{R}$ \\
\hline
\end{tabular}


TABLE 4-Continued

\begin{tabular}{|c|c|c|c|c|c|c|c|c|c|}
\hline ID & Confidence $^{\mathrm{b}}$ & Features $^{\mathrm{c}}$ & $\begin{array}{c}\text { RA } \\
{[\mathrm{J} 2000]}\end{array}$ & $\begin{array}{c}\text { Dec } \\
{[\mathrm{J} 2000]}\end{array}$ & $z_{p h o t}$ & $\begin{array}{c}\mathrm{M}_{r}^{\prime \mathrm{d}} \\
{[\mathrm{mag}]}\end{array}$ & $\begin{array}{c}\mathrm{M}_{\star}^{\mathrm{e}} \\
{\left[\log \left(\mathrm{M}_{\odot}\right)\right]}\end{array}$ & $\begin{array}{c}\left(g^{\prime}-r^{\prime}\right)^{\mathrm{f}} \\
{[\mathrm{mag}]}\end{array}$ & Red/Blue ${ }^{\mathrm{g}}$ \\
\hline W2-90 & 0 & - & 134.428360 & -5.262855 & 0.129 & -22.41 & 11.01 & 0.571 & $\mathrm{R}$ \\
\hline W2-91 & 0 & - & 134.675140 & -5.274775 & 0.076 & -21.04 & 10.13 & 0.274 & $\mathrm{~B}$ \\
\hline W2-92 & 0 & - & 134.526459 & -5.252233 & 0.144 & -22.94 & 11.26 & 0.598 & $\mathrm{R}$ \\
\hline W2-93 & 0 & - & 134.875931 & -5.188420 & 0.096 & -21.29 & 10.37 & 0.395 & B \\
\hline W2-94 & 1 & - & 134.426621 & -5.086775 & 0.094 & -21.83 & 10.70 & 0.500 & $\mathrm{~B}$ \\
\hline W2-96 & 0 & - & 134.323563 & -5.047715 & 0.065 & -20.64 & 10.37 & 0.625 & $\mathrm{R}$ \\
\hline W2-97 & 0 & - & 134.786636 & -5.043170 & 0.120 & -21.92 & 10.86 & 0.611 & $\mathrm{R}$ \\
\hline W2-99 & 0 & - & 133.990845 & -4.978526 & 0.153 & -23.58 & 11.52 & 0.605 & $\mathrm{R}$ \\
\hline W2-105 & 4 & $\mathrm{~S}$ & 134.349228 & -4.895570 & 0.095 & -21.98 & 10.92 & 0.644 & $\mathrm{R}$ \\
\hline W2-110 & 0 & - & 134.708542 & -4.756621 & 0.094 & -21.30 & 10.58 & 0.579 & $\mathrm{R}$ \\
\hline W2-111 & 0 & - & 134.085617 & -4.754487 & 0.119 & -22.01 & 10.90 & 0.610 & $\mathrm{R}$ \\
\hline W2-112 & 0 & - & 134.558044 & -4.724215 & 0.119 & -22.61 & 11.14 & 0.616 & $\mathrm{R}$ \\
\hline W2-113 & 2 & - & 134.839401 & -4.719500 & 0.116 & -22.01 & 10.88 & 0.594 & $\mathrm{R}$ \\
\hline W2-114 & 0 & - & 135.687607 & -5.668280 & 0.129 & -22.07 & 10.74 & 0.449 & B \\
\hline W2-115 & 0 & - & 135.053574 & -5.584150 & 0.082 & -21.11 & 10.44 & 0.520 & $\mathrm{R}$ \\
\hline W2-118 & 1 & - & 135.292801 & -5.354094 & 0.068 & -20.83 & 10.42 & 0.609 & $\mathrm{R}$ \\
\hline W2-120 & 0 & - & 135.755264 & -5.289909 & 0.180 & -23.09 & 11.31 & 0.597 & $\mathrm{R}$ \\
\hline W2-121 & 3 & $\mathrm{~S}$ & 135.812897 & -5.275785 & 0.074 & -20.86 & 10.38 & 0.557 & $\mathrm{R}$ \\
\hline W2-122 & 0 & - & 135.557220 & -5.214266 & 0.078 & -20.99 & 10.36 & 0.495 & $\mathrm{~B}$ \\
\hline W2-123 & 0 & - & 134.986710 & -5.150891 & 0.099 & -21.49 & 10.47 & 0.416 & $\mathrm{~B}$ \\
\hline W2-124 & 0 & - & 135.593979 & -5.093661 & 0.084 & -21.83 & 10.76 & 0.553 & $\mathrm{R}$ \\
\hline W2-125 & 4 & $\mathrm{~S}$ & 134.981278 & -5.050979 & 0.095 & -21.50 & 10.61 & 0.537 & $\mathrm{R}$ \\
\hline W2-128 & 4 & $\mathrm{~F}$ & 135.198120 & -4.994212 & 0.181 & -22.92 & 11.28 & 0.631 & $\mathrm{R}$ \\
\hline W2-133 & 0 & - & 135.290176 & -4.718444 & 0.139 & -22.39 & 11.01 & 0.573 & $\mathrm{R}$ \\
\hline W2-134 & 0 & - & 135.987289 & -5.535717 & 0.134 & -22.39 & 10.93 & 0.503 & $\mathrm{~B}$ \\
\hline W2-135 & 0 & - & 135.999420 & -5.529022 & 0.141 & -22.46 & 11.03 & 0.561 & $\mathrm{R}$ \\
\hline W2-136 & 2 & - & 136.320755 & -5.473670 & 0.150 & -22.52 & 11.11 & 0.615 & $\mathrm{R}$ \\
\hline W2-137 & 0 & - & 135.926346 & -5.412499 & 0.180 & -22.92 & 11.27 & 0.619 & $\mathrm{R}$ \\
\hline W2-139 & 2 & - & 136.241318 & -5.355691 & 0.121 & -21.97 & 10.91 & 0.633 & $\mathrm{R}$ \\
\hline W2-140 & 0 & - & 136.099869 & -5.212903 & 0.092 & -21.25 & 10.45 & 0.482 & $\mathrm{~B}$ \\
\hline W2-143 & 0 & - & 136.468353 & -5.018272 & 0.129 & -22.55 & 11.09 & 0.590 & $\mathrm{R}$ \\
\hline W2-145 & 0 & - & 136.124847 & -4.951866 & 0.083 & -21.02 & 10.15 & 0.300 & $\mathrm{~B}$ \\
\hline W2-146 & 1 & - & 136.312515 & -4.889222 & 0.090 & -21.82 & 10.83 & 0.618 & $\mathrm{R}$ \\
\hline W2-147 & 1 & - & 132.516892 & -4.674360 & 0.082 & -20.89 & 10.21 & 0.398 & $\mathrm{~B}$ \\
\hline W2-152 & 0 & - & 132.302368 & -4.405945 & 0.095 & -21.34 & 10.36 & 0.368 & $\mathrm{~B}$ \\
\hline W2-154 & 0 & - & 132.527802 & -4.330365 & 0.126 & -22.34 & 10.87 & 0.467 & $\mathrm{~B}$ \\
\hline W2-156 & 0 & - & 132.175110 & -4.326365 & 0.192 & -23.04 & 11.32 & 0.621 & $\mathrm{R}$ \\
\hline W2-157 & 0 & - & 132.787613 & -4.175069 & 0.192 & -23.42 & 11.33 & 0.493 & $\mathrm{~B}$ \\
\hline W2-158 & 0 & - & 132.804611 & -4.138937 & 0.125 & -22.09 & 10.83 & 0.522 & $\mathrm{~B}$ \\
\hline W2-159 & 0 & - & 132.889358 & -4.113917 & 0.145 & -22.69 & 11.16 & 0.599 & $\mathrm{R}$ \\
\hline W2-160 & 0 & - & 132.731720 & -4.100234 & 0.138 & -22.23 & 10.98 & 0.605 & $\mathrm{R}$ \\
\hline W2-163 & 0 & - & 132.305405 & -3.988713 & 0.084 & -21.21 & 10.54 & 0.571 & $\mathrm{R}$ \\
\hline W2-169 & 2 & - & 133.395615 & -4.607748 & 0.067 & -20.73 & 10.30 & 0.530 & $\mathrm{R}$ \\
\hline W2-170 & 4 & $\mathrm{~L}$ & 133.407410 & -4.602370 & 0.046 & -19.79 & 9.95 & 0.553 & $\mathrm{R}$ \\
\hline
\end{tabular}


TABLE 4-Continued

\begin{tabular}{|c|c|c|c|c|c|c|c|c|c|}
\hline ID & Confidence $^{\mathrm{b}}$ & Features $^{\mathrm{c}}$ & $\begin{array}{c}\text { RA } \\
{[\mathrm{J} 2000]}\end{array}$ & $\begin{array}{c}\text { Dec } \\
{[\mathrm{J} 2000]}\end{array}$ & $z_{p h o t}$ & $\begin{array}{c}\mathrm{M}_{r}^{\prime \mathrm{d}} \\
{[\mathrm{mag}]}\end{array}$ & $\begin{array}{c}\mathrm{M}_{\star}^{\mathrm{e}} \\
{\left[\log \left(\mathrm{M}_{\odot}\right)\right]}\end{array}$ & $\begin{array}{c}\left(g^{\prime}-r^{\prime}\right)^{\mathrm{f}} \\
{[\mathrm{mag}]}\end{array}$ & Red/Blue ${ }^{\mathrm{g}}$ \\
\hline W2-171 & 0 & - & 133.442429 & -4.486828 & 0.132 & -22.65 & 11.16 & 0.617 & $\mathrm{R}$ \\
\hline W2-172 & 1 & - & 133.775757 & -4.406029 & 0.065 & -21.09 & 10.24 & 0.354 & $\mathrm{~B}$ \\
\hline W2-173 & 0 & - & 133.601166 & -4.379664 & 0.074 & -20.74 & 9.92 & 0.188 & $\mathrm{~B}$ \\
\hline W2-174 & 3 & $\mathrm{~F}$ & 133.640060 & -4.101349 & 0.060 & -20.26 & 9.66 & 0.131 & B \\
\hline W2-176 & 0 & - & 133.310883 & -3.883724 & 0.089 & -21.63 & 10.48 & 0.368 & $\mathrm{~B}$ \\
\hline W2-179 & 0 & - & 134.109619 & -4.629538 & 0.071 & -20.95 & 10.23 & 0.390 & $\mathrm{~B}$ \\
\hline W2-180 & 0 & - & 134.482361 & -4.607965 & 0.064 & -20.75 & 10.06 & 0.313 & $\mathrm{~B}$ \\
\hline W2-181 & 0 & - & 134.342041 & -4.608329 & 0.120 & -21.97 & 10.88 & 0.613 & $\mathrm{R}$ \\
\hline W2-182 & 0 & - & 134.737427 & -4.563935 & 0.151 & -23.21 & 11.39 & 0.618 & $\mathrm{R}$ \\
\hline W2-183 & 0 & - & 134.836960 & -4.544373 & 0.057 & -21.11 & 10.02 & 0.148 & $\mathrm{~B}$ \\
\hline W2-184 & 0 & - & 134.498932 & -4.542769 & 0.117 & -22.29 & 10.74 & 0.367 & $\mathrm{~B}$ \\
\hline W2-188 & 1 & - & 134.077087 & -4.343590 & 0.154 & -22.69 & 11.12 & 0.567 & $\mathrm{R}$ \\
\hline W2-190 & 4 & M & 134.182358 & -4.220983 & 0.180 & -22.93 & 11.24 & 0.592 & $\mathrm{R}$ \\
\hline W2-192 & 0 & - & 134.123688 & -4.147353 & 0.081 & -21.43 & 10.39 & 0.361 & B \\
\hline W2-193 & 2 & - & 134.718765 & -4.170416 & 0.104 & -21.80 & 10.69 & 0.496 & $\mathrm{~B}$ \\
\hline W2-195 & 0 & - & 134.002884 & -4.108618 & 0.086 & -21.40 & 10.47 & 0.445 & $\mathrm{~B}$ \\
\hline W2-196 & 0 & - & 134.065872 & -4.033625 & 0.147 & -22.85 & 11.21 & 0.594 & $\mathrm{R}$ \\
\hline W2-200 & 0 & - & 135.343567 & -4.670206 & 0.090 & -21.18 & 10.58 & 0.622 & $\mathrm{R}$ \\
\hline W2-202 & 0 & - & 135.494507 & -4.618280 & 0.073 & -20.80 & 10.32 & 0.528 & $\mathrm{R}$ \\
\hline W2-203 & 0 & - & 135.814468 & -4.608713 & 0.150 & -22.68 & 11.15 & 0.598 & $\mathrm{R}$ \\
\hline W2-204 & 4 & $\mathrm{~A}$ & 135.184586 & -4.595252 & 0.150 & -22.63 & 11.18 & 0.641 & $\mathrm{R}$ \\
\hline W2-207 & 0 & - & 135.829803 & -4.548098 & 0.188 & -23.06 & 11.34 & 0.627 & $\mathrm{R}$ \\
\hline W2-210 & 0 & - & 135.021881 & -4.459963 & 0.106 & -22.15 & 10.90 & 0.559 & $\mathrm{R}$ \\
\hline W2-211 & 0 & - & 135.588928 & -4.460535 & 0.090 & -21.19 & 10.60 & 0.634 & $\mathrm{R}$ \\
\hline W2-213 & 0 & - & 135.479981 & -4.429192 & 0.090 & -21.22 & 10.55 & 0.585 & $\mathrm{R}$ \\
\hline W2-215 & 0 & - & 135.259399 & -4.396011 & 0.162 & -23.06 & 11.33 & 0.621 & $\mathrm{R}$ \\
\hline W2-216 & 0 & - & 134.994598 & -4.369384 & 0.180 & -23.30 & 11.44 & 0.639 & $\mathrm{R}$ \\
\hline W2-218 & 0 & - & 135.248123 & -4.217878 & 0.105 & -21.80 & 10.75 & 0.555 & $\mathrm{R}$ \\
\hline W2-219 & 0 & - & 134.998444 & -4.188590 & 0.103 & -21.98 & 10.43 & 0.202 & $\mathrm{~B}$ \\
\hline W2-220 & 2 & - & 134.931320 & -4.192882 & 0.164 & -22.90 & 11.29 & 0.641 & $\mathrm{R}$ \\
\hline W2-221 & 0 & - & 135.061218 & -4.141319 & 0.090 & -21.38 & 10.55 & 0.526 & $\mathrm{R}$ \\
\hline W2-222 & 1 & - & 135.095703 & -4.120724 & 0.126 & -22.47 & 10.99 & 0.530 & $\mathrm{~B}$ \\
\hline W2-224 & 0 & - & 135.039917 & -4.100404 & 0.167 & -23.19 & 11.39 & 0.629 & $\mathrm{R}$ \\
\hline W2-226 & 0 & - & 135.048904 & -4.013731 & 0.153 & -22.80 & 11.19 & 0.590 & $\mathrm{R}$ \\
\hline W2-227 & 1 & - & 135.229477 & -3.986971 & 0.145 & -22.64 & 10.74 & 0.246 & $\mathrm{~B}$ \\
\hline W2-231 & 0 & - & 136.716858 & -4.619988 & 0.169 & -23.00 & 11.27 & 0.588 & $\mathrm{R}$ \\
\hline W2-232 & 0 & - & 136.398773 & -4.613728 & 0.131 & -22.33 & 11.05 & 0.634 & $\mathrm{R}$ \\
\hline W2-234 & 0 & - & 136.051926 & -4.373498 & 0.130 & -22.82 & 11.25 & 0.636 & $\mathrm{R}$ \\
\hline W2-235 & 0 & - & 135.990906 & -4.301221 & 0.134 & -22.37 & 11.04 & 0.606 & $\mathrm{R}$ \\
\hline W2-238 & 0 & - & 136.584213 & -4.188684 & 0.101 & -21.49 & 10.70 & 0.621 & $\mathrm{R}$ \\
\hline W2-240 & 0 & - & 136.581207 & -4.017592 & 0.083 & -21.86 & 10.77 & 0.553 & $\mathrm{R}$ \\
\hline W2-241 & 0 & - & 136.666214 & -4.009181 & 0.156 & -22.58 & 10.63 & 0.172 & B \\
\hline W2-242 & 0 & - & 135.934112 & -3.931017 & 0.163 & -22.95 & 11.31 & 0.645 & $\mathrm{R}$ \\
\hline W2-245 & 1 & - & 132.611786 & -3.303008 & 0.107 & -21.81 & 10.55 & 0.376 & $\mathrm{~B}$ \\
\hline
\end{tabular}


TABLE 4-Continued

\begin{tabular}{|c|c|c|c|c|c|c|c|c|c|}
\hline ID & Confidence $^{\mathrm{b}}$ & Features $^{\mathrm{c}}$ & $\begin{array}{c}\text { RA } \\
{[\mathrm{J} 2000]}\end{array}$ & $\begin{array}{c}\text { Dec } \\
{[\mathrm{J} 2000]}\end{array}$ & $z_{p h o t}$ & $\begin{array}{c}\mathrm{M}_{r}^{\prime \mathrm{d}} \\
{[\mathrm{mag}]}\end{array}$ & $\begin{array}{c}\mathrm{M}_{\star}^{\mathrm{e}} \\
{\left[\log \left(\mathrm{M}_{\odot}\right)\right]}\end{array}$ & $\begin{array}{c}\left(g^{\prime}-r^{\prime}\right)^{\mathrm{f}} \\
{[\mathrm{mag}]}\end{array}$ & Red/Blue ${ }^{\mathrm{g}}$ \\
\hline W2-246 & 0 & - & 132.380005 & -3.304142 & 0.065 & -20.49 & 10.19 & 0.524 & $\mathrm{R}$ \\
\hline W2-252 & 3 & M & 133.701965 & -3.148548 & 0.111 & -22.42 & 11.03 & 0.578 & $\mathrm{R}$ \\
\hline W2-253 & 3 & $\mathrm{~A}$ & 133.198395 & -3.092494 & 0.060 & -21.28 & 10.65 & 0.650 & $\mathrm{R}$ \\
\hline W2-254 & 1 & - & 133.253647 & -3.035109 & 0.091 & -21.86 & 10.85 & 0.625 & $\mathrm{R}$ \\
\hline W2-260 & 0 & - & 134.592590 & -3.565807 & 0.182 & -23.45 & 11.48 & 0.613 & $\mathrm{R}$ \\
\hline W2-262 & 0 & - & 134.759598 & -3.083574 & 0.144 & -22.59 & 10.99 & 0.491 & $\mathrm{~B}$ \\
\hline W2-264 & 3 & $\mathrm{~S}$ & 134.600677 & -3.026728 & 0.079 & -20.98 & 10.34 & 0.475 & B \\
\hline W2-266 & 1 & - & 134.304062 & -2.872144 & 0.134 & -22.34 & 10.99 & 0.576 & $\mathrm{R}$ \\
\hline W2-271 & 0 & - & 135.732040 & -3.453702 & 0.064 & -20.76 & 10.31 & 0.527 & $\mathrm{R}$ \\
\hline W2-272 & 0 & - & 134.988358 & -3.436502 & 0.067 & -20.56 & 10.21 & 0.519 & $\mathrm{R}$ \\
\hline W2-273 & 0 & - & 135.148850 & -3.420034 & 0.179 & -23.01 & 11.34 & 0.643 & $\mathrm{R}$ \\
\hline W2-274 & 0 & - & 135.237701 & -3.397559 & 0.153 & -22.95 & 11.29 & 0.627 & $\mathrm{R}$ \\
\hline W2-283 & 2 & - & 135.975525 & -3.760420 & 0.087 & -21.71 & 10.64 & 0.485 & $\mathrm{~B}$ \\
\hline W2-285 & 0 & - & 136.415604 & -3.701932 & 0.128 & -23.11 & 11.36 & 0.626 & $\mathrm{R}$ \\
\hline W2-288 & 0 & - & 136.535477 & -3.297325 & 0.187 & -23.34 & 10.87 & 0.106 & $\mathrm{~B}$ \\
\hline W2-289 & 0 & - & 136.346664 & -3.095819 & 0.090 & -21.33 & 10.40 & 0.411 & $\mathrm{~B}$ \\
\hline W2-298 & 1 & - & 132.558639 & -2.512032 & 0.125 & -22.05 & 10.43 & 0.179 & $\mathrm{~B}$ \\
\hline W2-299 & 3 & $\mathrm{~S}, \mathrm{~L}$ & 132.204147 & -2.317823 & 0.100 & -22.07 & 10.70 & 0.417 & $\mathrm{~B}$ \\
\hline W2-300 & 0 & - & 132.264084 & -2.186942 & 0.075 & -20.73 & 10.29 & 0.528 & $\mathrm{R}$ \\
\hline W2-303 & 3 & M & 132.347031 & -2.104322 & 0.086 & -21.18 & 10.48 & 0.533 & $\mathrm{R}$ \\
\hline W2-304 & 0 & - & 132.341003 & -2.086731 & 0.107 & -21.63 & 10.74 & 0.606 & $\mathrm{R}$ \\
\hline W2-305 & 0 & - & 132.714615 & -2.051213 & 0.061 & -20.40 & 10.30 & 0.652 & $\mathrm{R}$ \\
\hline W2-319 & 1 & - & 135.001083 & -2.756109 & 0.096 & -21.43 & 10.52 & 0.481 & B \\
\hline W2-321 & 0 & - & 135.463547 & -2.664347 & 0.143 & -22.49 & 11.07 & 0.595 & $\mathrm{R}$ \\
\hline W2-322 & 3 & $\mathrm{~F}$ & 135.711639 & -2.611654 & 0.112 & -21.92 & 10.83 & 0.580 & $\mathrm{R}$ \\
\hline W2-323 & 0 & - & 135.584045 & -2.439234 & 0.196 & -23.12 & 11.33 & 0.599 & $\mathrm{R}$ \\
\hline W2-329 & 1 & - & 135.159592 & -2.046415 & 0.135 & -22.28 & 10.98 & 0.591 & $\mathrm{R}$ \\
\hline W2-333 & 0 & - & 136.102127 & -2.623159 & 0.122 & -21.88 & 10.85 & 0.612 & $\mathrm{R}$ \\
\hline W2-335 & 1 & - & 136.207016 & -2.571914 & 0.108 & -21.74 & 10.78 & 0.606 & $\mathrm{R}$ \\
\hline W2-336 & 0 & - & 136.776611 & -2.564915 & 0.149 & -22.55 & 11.06 & 0.563 & $\mathrm{R}$ \\
\hline W2-338 & 0 & - & 136.518890 & -2.467287 & 0.150 & -22.62 & 11.13 & 0.600 & $\mathrm{R}$ \\
\hline W2-341 & 1 & - & 135.909988 & -2.330835 & 0.083 & -21.16 & 10.45 & 0.515 & $\mathrm{~B}$ \\
\hline W2-343 & 0 & - & 136.003708 & -2.278247 & 0.156 & -22.95 & 11.27 & 0.608 & $\mathrm{R}$ \\
\hline W2-344 & 2 & - & 136.748779 & -2.154649 & 0.152 & -23.18 & 11.36 & 0.599 & $\mathrm{R}$ \\
\hline W2-345 & 0 & - & 132.321686 & -1.756479 & 0.077 & -21.53 & 10.46 & 0.395 & $\mathrm{~B}$ \\
\hline W2-346 & 0 & - & 132.929489 & -1.555087 & 0.065 & -20.59 & 10.39 & 0.660 & $\mathrm{R}$ \\
\hline W2-348 & 3 & $\mathrm{~A}$ & 132.477875 & -1.434345 & 0.134 & -22.19 & 10.54 & 0.224 & B \\
\hline W2-351 & 4 & $\mathrm{~S}, \mathrm{~F}$ & 133.975159 & -1.899000 & 0.079 & -21.66 & 10.59 & 0.456 & $\mathrm{~B}$ \\
\hline W2-353 & 0 & - & 133.381226 & -1.699979 & 0.116 & -22.20 & 10.95 & 0.588 & $\mathrm{R}$ \\
\hline W2-355 & 4 & $\mathrm{~A}$ & 133.831940 & -1.682380 & 0.095 & -21.28 & 10.60 & 0.605 & $\mathrm{R}$ \\
\hline W2-356 & 1 & - & 133.312698 & -1.648569 & 0.158 & -23.17 & 11.21 & 0.479 & $\mathrm{~B}$ \\
\hline W2-357 & 0 & - & 133.284500 & -1.647511 & 0.160 & -22.69 & 11.01 & 0.468 & B \\
\hline W2-359 & 0 & - & 133.758240 & -1.338264 & 0.073 & -20.90 & 10.21 & 0.389 & $\mathrm{~B}$ \\
\hline W2-367 & 0 & - & 134.482437 & -1.490402 & 0.127 & -21.90 & 10.50 & 0.296 & $\mathrm{~B}$ \\
\hline
\end{tabular}


TABLE 4-Continued

\begin{tabular}{|c|c|c|c|c|c|c|c|c|c|}
\hline ID & Confidence $^{\mathrm{b}}$ & Features $^{c}$ & $\begin{array}{c}\text { RA } \\
{[\mathrm{J} 2000]}\end{array}$ & $\begin{array}{c}\text { Dec } \\
{[\mathrm{J} 2000]}\end{array}$ & $z_{\text {phot }}$ & $\begin{array}{c}\mathrm{M}_{r}^{\prime \mathrm{d}} \\
{[\mathrm{mag}]}\end{array}$ & $\begin{array}{c}\mathrm{M}_{\star}{ }^{\mathrm{e}} \\
{\left[\log \left(\mathrm{M}_{\odot}\right)\right]}\end{array}$ & $\begin{array}{c}\left(g^{\prime}-r^{\prime}\right)^{\mathrm{f}} \\
{[\mathrm{mag}]}\end{array}$ & Red/Blue ${ }^{\mathrm{g}}$ \\
\hline W2-368 & 0 & - & 134.066177 & -1.477762 & 0.134 & -22.30 & 10.74 & 0.364 & B \\
\hline W2-369 & 4 & $\mathrm{~S}$ & 134.375290 & -1.393179 & 0.060 & -20.59 & 10.32 & 0.603 & $\mathrm{R}$ \\
\hline W2-376 & 0 & - & 134.140808 & -0.982172 & 0.090 & -21.20 & 10.58 & 0.617 & $\mathrm{R}$ \\
\hline W2-377 & 0 & - & 135.488373 & -1.913194 & 0.077 & -20.87 & 10.41 & 0.583 & $\mathrm{R}$ \\
\hline W2-382 & 2 & - & 135.470764 & -1.443076 & 0.177 & -23.30 & 11.40 & 0.598 & $\mathrm{R}$ \\
\hline W2-383 & 0 & - & 134.959381 & -1.356144 & 0.172 & -22.95 & 11.28 & 0.613 & $\mathrm{R}$ \\
\hline W2-384 & 0 & - & 135.347717 & -1.266364 & 0.079 & -20.87 & 10.19 & 0.387 & B \\
\hline W2-385 & 4 & $\mathrm{~L}$ & 135.601990 & -1.262184 & 0.097 & -21.73 & 10.72 & 0.554 & $\mathrm{R}$ \\
\hline W2-392 & 0 & - & 136.503021 & -1.555141 & 0.115 & -22.05 & 10.89 & 0.588 & $\mathrm{R}$ \\
\hline W2-393 & 0 & - & 136.488342 & -1.530554 & 0.086 & -21.52 & 10.41 & 0.348 & B \\
\hline W2-394 & 1 & - & 136.606049 & -1.406790 & 0.136 & -23.04 & 11.15 & 0.468 & B \\
\hline W2-395 & 0 & - & 136.289154 & -1.181990 & 0.180 & -23.23 & 11.36 & 0.588 & $\mathrm{R}$ \\
\hline W2-396 & 4 & $\mathrm{H}, \mathrm{F}$ & 136.284973 & -1.148197 & 0.063 & -21.21 & 10.56 & 0.596 & $\mathrm{R}$ \\
\hline W2-397 & 4 & $\mathrm{~A}, \mathrm{~L}$ & 136.328873 & -1.149851 & 0.089 & -21.06 & 10.26 & 0.381 & B \\
\hline W2-399 & 0 & - & 136.787796 & -1.057288 & 0.067 & -20.53 & 10.18 & 0.502 & B \\
\hline W2-400 & 0 & - & 136.018036 & -1.033201 & 0.152 & -22.91 & 11.06 & 0.429 & B \\
\hline W3-2 & 0 & - & 209.808914 & 51.244030 & 0.057 & -20.97 & 10.47 & 0.596 & $\mathrm{R}$ \\
\hline W3-3 & 0 & - & 210.462769 & 51.332729 & 0.086 & -21.30 & 10.60 & 0.599 & $\mathrm{R}$ \\
\hline W3-4 & 3 & M & 209.485474 & 51.354378 & 0.180 & -22.96 & 11.29 & 0.623 & $\mathrm{R}$ \\
\hline W3-5 & 0 & - & 209.821686 & 51.385986 & 0.191 & -23.22 & 11.41 & 0.634 & $\mathrm{R}$ \\
\hline W3-7 & 0 & - & 210.130035 & 51.642860 & 0.153 & -22.44 & 10.81 & 0.379 & B \\
\hline W3-8 & 0 & - & 210.094452 & 51.859917 & 0.165 & -23.34 & 11.19 & 0.396 & B \\
\hline W3-9 & 1 & - & 209.938370 & 52.025921 & 0.180 & -22.96 & 11.31 & 0.639 & $\mathrm{R}$ \\
\hline W3-12 & 1 & - & 210.942337 & 51.332611 & 0.083 & -21.14 & 10.31 & 0.398 & B \\
\hline W3-13 & 4 & $\mathrm{~S}$ & 211.455597 & 51.391953 & 0.138 & -22.66 & 11.09 & 0.545 & $\mathrm{R}$ \\
\hline W3-14 & 4 & $\mathrm{~A}$ & 212.006683 & 51.707348 & 0.076 & -20.86 & 10.42 & 0.598 & $\mathrm{R}$ \\
\hline W3-16 & 2 & - & 211.849899 & 51.834339 & 0.187 & -23.10 & 11.37 & 0.641 & $\mathrm{R}$ \\
\hline W3-18 & 1 & - & 211.486053 & 51.917835 & 0.100 & -21.97 & 10.64 & 0.392 & B \\
\hline W3-19 & 0 & - & 211.724411 & 52.029446 & 0.077 & -21.08 & 10.21 & 0.331 & B \\
\hline W3-20 & 0 & - & 211.034622 & 52.074902 & 0.084 & -21.20 & 10.35 & 0.413 & B \\
\hline W3-22 & 0 & - & 212.357788 & 51.231632 & 0.150 & -23.18 & 11.36 & 0.602 & $\mathrm{R}$ \\
\hline W3-23 & 0 & - & 212.391144 & 51.295437 & 0.150 & -22.46 & 11.12 & 0.648 & $\mathrm{R}$ \\
\hline W3-24 & 0 & - & 212.435638 & 51.484604 & 0.183 & -23.28 & 11.42 & 0.625 & $\mathrm{R}$ \\
\hline W3-26 & 0 & - & 212.625275 & 52.130371 & 0.124 & -21.92 & 10.63 & 0.408 & B \\
\hline W3-28 & 0 & - & 213.814575 & 51.231358 & 0.120 & -21.97 & 10.91 & 0.640 & $\mathrm{R}$ \\
\hline W3-29 & 0 & - & 213.848114 & 51.443409 & 0.063 & -21.73 & 10.80 & 0.623 & $\mathrm{R}$ \\
\hline W3-31 & 0 & - & 214.099060 & 51.482929 & 0.140 & -22.64 & 11.15 & 0.615 & $\mathrm{R}$ \\
\hline W3-32 & 1 & - & 213.784790 & 51.613769 & 0.062 & -20.74 & 10.23 & 0.465 & B \\
\hline W3-33 & 0 & - & 214.616455 & 51.634003 & 0.090 & -21.52 & 10.51 & 0.435 & B \\
\hline W3-34 & 0 & - & 213.963867 & 51.856842 & 0.065 & -20.82 & 10.36 & 0.559 & $\mathrm{R}$ \\
\hline W3-35 & 1 & - & 213.896790 & 51.864822 & 0.090 & -21.47 & 10.70 & 0.624 & $\mathrm{R}$ \\
\hline W3-36 & 0 & - & 214.878281 & 51.924484 & 0.111 & -22.31 & 10.54 & 0.182 & B \\
\hline W3-38 & 0 & - & 214.745422 & 52.028076 & 0.092 & -21.71 & 10.75 & 0.585 & $\mathrm{R}$ \\
\hline W3-39 & 4 & - & 213.968430 & 52.052456 & 0.096 & -21.85 & 10.72 & 0.510 & B \\
\hline
\end{tabular}


TABLE 4-Continued

\begin{tabular}{|c|c|c|c|c|c|c|c|c|c|}
\hline ID & Confidence $^{\mathrm{b}}$ & Features $^{\mathrm{c}}$ & $\begin{array}{c}\text { RA } \\
{[\mathrm{J} 2000]}\end{array}$ & $\begin{array}{c}\text { Dec } \\
{[\mathrm{J} 2000]}\end{array}$ & $z_{\text {phot }}$ & $\begin{array}{c}\mathrm{M}_{r}^{\prime \mathrm{d}} \\
{[\mathrm{mag}]}\end{array}$ & $\begin{array}{c}\mathrm{M}_{\star}^{\mathrm{e}} \\
{\left[\log \left(\mathrm{M}_{\odot}\right)\right]}\end{array}$ & $\begin{array}{c}\left(g^{\prime}-r^{\prime}\right)^{\mathrm{f}} \\
{[\mathrm{mag}]}\end{array}$ & Red/Blue ${ }^{\mathrm{g}}$ \\
\hline W3-40 & 1 & - & 213.995758 & 52.050819 & 0.086 & -21.62 & 10.54 & 0.435 & B \\
\hline W3-41 & 1 & - & 213.957642 & 52.054176 & 0.089 & -21.54 & 10.61 & 0.516 & B \\
\hline W3-43 & 2 & - & 213.765137 & 52.076160 & 0.154 & -23.05 & 11.26 & 0.561 & $\mathrm{R}$ \\
\hline W3-45 & 4 & $\mathrm{~F}$ & 213.817093 & 52.082340 & 0.090 & -21.25 & 10.58 & 0.600 & $\mathrm{R}$ \\
\hline W3-48 & 0 & - & 214.609482 & 52.147411 & 0.133 & -22.03 & 10.63 & 0.367 & $\mathrm{~B}$ \\
\hline W3-49 & 0 & - & 214.050446 & 52.160267 & 0.061 & -20.53 & 10.29 & 0.595 & $\mathrm{R}$ \\
\hline W3-50 & 1 & - & 215.867218 & 51.330772 & 0.072 & -20.65 & 10.09 & 0.371 & $\mathrm{~B}$ \\
\hline W3-56 & 1 & - & 215.869675 & 51.918484 & 0.099 & -21.38 & 10.67 & 0.634 & $\mathrm{R}$ \\
\hline W3-58 & 0 & - & 215.896591 & 52.154884 & 0.062 & -20.71 & 10.32 & 0.555 & $\mathrm{R}$ \\
\hline W3-60 & 4 & $\mathrm{~S}, \mathrm{~L}, \mathrm{~F}$ & 217.666061 & 51.258587 & 0.180 & -22.91 & 11.27 & 0.619 & $\mathrm{R}$ \\
\hline W3-61 & 0 & - & 216.790588 & 51.329815 & 0.107 & -22.30 & 10.77 & 0.396 & $\mathrm{~B}$ \\
\hline W3-63 & 0 & - & 217.458588 & 51.692394 & 0.090 & -21.81 & 10.45 & 0.284 & $\mathrm{~B}$ \\
\hline W3-64 & 0 & - & 217.168594 & 51.722370 & 0.091 & -21.76 & 10.47 & 0.314 & $\mathrm{~B}$ \\
\hline W3-66 & 3 & M & 217.373825 & 51.741810 & 0.089 & -21.08 & 10.22 & 0.340 & B \\
\hline W3-67 & 4 & - & 217.168747 & 52.056721 & 0.095 & -22.09 & 10.95 & 0.627 & $\mathrm{R}$ \\
\hline W3-70 & 0 & - & 218.759338 & 51.467274 & 0.074 & -21.17 & 10.55 & 0.595 & $\mathrm{R}$ \\
\hline W3-72 & 0 & - & 218.496979 & 51.510536 & 0.076 & -21.41 & 10.66 & 0.614 & $\mathrm{R}$ \\
\hline W3-73 & 0 & - & 218.268997 & 51.522354 & 0.167 & -22.97 & 11.10 & 0.449 & $\mathrm{~B}$ \\
\hline W3-74 & 0 & - & 219.370926 & 51.582718 & 0.060 & -20.27 & 10.21 & 0.618 & $\mathrm{R}$ \\
\hline W3-75 & 3 & $\mathrm{~A}$ & 219.326599 & 51.612659 & 0.156 & -22.98 & 11.29 & 0.615 & $\mathrm{R}$ \\
\hline W3-76 & 0 & - & 219.349945 & 51.646694 & 0.152 & -22.59 & 10.66 & 0.185 & $\mathrm{~B}$ \\
\hline W3-77 & 0 & - & 219.128418 & 51.660389 & 0.075 & -20.71 & 10.07 & 0.334 & $\mathrm{~B}$ \\
\hline W3-78 & 1 & - & 218.992279 & 51.692577 & 0.075 & -21.32 & 10.36 & 0.373 & B \\
\hline W3-79 & 0 & - & 219.359604 & 51.694965 & 0.088 & -21.54 & 10.47 & 0.397 & $\mathrm{~B}$ \\
\hline W3-80 & 0 & - & 219.439178 & 51.735279 & 0.087 & -21.14 & 10.26 & 0.352 & $\mathrm{~B}$ \\
\hline W3-81 & 2 & - & 219.424499 & 51.763504 & 0.125 & -21.98 & 10.66 & 0.411 & $\mathrm{~B}$ \\
\hline W3-82 & 4 & M & 218.930649 & 51.769669 & 0.053 & -20.19 & 10.13 & 0.576 & $\mathrm{R}$ \\
\hline W3-83 & 1 & - & 219.180328 & 51.791294 & 0.150 & -22.93 & 11.29 & 0.629 & $\mathrm{R}$ \\
\hline W3-84 & 0 & - & 219.410309 & 51.792931 & 0.107 & -21.48 & 10.32 & 0.287 & $\mathrm{~B}$ \\
\hline W3-85 & 0 & - & 219.153854 & 51.920685 & 0.090 & -21.42 & 10.68 & 0.626 & $\mathrm{R}$ \\
\hline W3-86 & 0 & - & 218.736588 & 51.970367 & 0.088 & -21.27 & 10.32 & 0.358 & $\mathrm{~B}$ \\
\hline W3-88 & 1 & - & 218.435577 & 51.974934 & 0.091 & -21.22 & 10.60 & 0.622 & $\mathrm{R}$ \\
\hline W3-89 & 0 & - & 218.426041 & 52.056137 & 0.075 & -21.06 & 10.25 & 0.369 & B \\
\hline W3-91 & 3 & M & 210.215012 & 52.268498 & 0.150 & -23.10 & 11.36 & 0.636 & $\mathrm{R}$ \\
\hline W3-92 & 0 & - & 210.487763 & 52.279442 & 0.136 & -22.65 & 10.89 & 0.377 & $\mathrm{~B}$ \\
\hline W3-93 & 0 & - & 209.798569 & 52.362545 & 0.162 & -23.01 & 10.91 & 0.260 & B \\
\hline W3-94 & 1 & - & 209.292969 & 52.343117 & 0.175 & -22.77 & 11.06 & 0.482 & B \\
\hline W3-95 & 3 & $\mathrm{~F}, \mathrm{H}$ & 209.650284 & 52.355919 & 0.117 & -21.95 & 10.60 & 0.364 & $\mathrm{~B}$ \\
\hline W3-96 & 0 & - & 209.666946 & 52.360199 & 0.150 & -22.68 & 11.23 & 0.668 & $\mathrm{R}$ \\
\hline W3-97 & 1 & - & 209.940918 & 52.376900 & 0.140 & -22.17 & 10.74 & 0.417 & $\mathrm{~B}$ \\
\hline W3-98 & 0 & - & 209.355331 & 52.392796 & 0.127 & -22.34 & 11.08 & 0.653 & $\mathrm{R}$ \\
\hline W3-99 & 0 & - & 209.590347 & 52.392918 & 0.066 & -20.76 & 10.03 & 0.280 & B \\
\hline W3-101 & 0 & - & 210.164871 & 52.439419 & 0.150 & -22.71 & 11.20 & 0.632 & $\mathrm{R}$ \\
\hline W3-102 & 4 & $\mathrm{~S}$ & 210.275452 & 52.472275 & 0.090 & -21.24 & 10.61 & 0.631 & $\mathrm{R}$ \\
\hline
\end{tabular}


TABLE 4-Continued

\begin{tabular}{|c|c|c|c|c|c|c|c|c|c|}
\hline ID & Confidence $^{\mathrm{b}}$ & Features $^{c}$ & $\begin{array}{c}\mathrm{RA} \\
{[\mathrm{J} 2000]}\end{array}$ & $\begin{array}{c}\text { Dec } \\
{[\mathrm{J} 2000]}\end{array}$ & $z_{p h o t}$ & $\begin{array}{c}\mathrm{M}_{r}^{\prime \mathrm{d}} \\
{[\mathrm{mag}]}\end{array}$ & $\begin{array}{c}\mathrm{M}_{\star}^{\mathrm{e}} \\
{\left[\log \left(\mathrm{M}_{\odot}\right)\right]}\end{array}$ & $\begin{array}{c}\left(g^{\prime}-r^{\prime}\right)^{\mathrm{f}} \\
{[\mathrm{mag}]}\end{array}$ & Red/Blue ${ }^{\mathrm{g}}$ \\
\hline W3-103 & 0 & - & 209.749939 & 52.501736 & 0.181 & -22.85 & 11.03 & 0.431 & $\mathrm{~B}$ \\
\hline W3-104 & 0 & - & 210.152359 & 52.538620 & 0.073 & -21.60 & 10.41 & 0.321 & $\mathrm{~B}$ \\
\hline W3-106 & 0 & - & 209.535187 & 52.526508 & 0.104 & -21.50 & 10.44 & 0.378 & $\mathrm{~B}$ \\
\hline W3-107 & 0 & - & 209.404678 & 52.580059 & 0.117 & -21.83 & 10.86 & 0.645 & $\mathrm{R}$ \\
\hline W3-108 & 3 & M & 209.618195 & 52.586479 & 0.060 & -20.34 & 10.26 & 0.642 & $\mathrm{R}$ \\
\hline W3-110 & 1 & - & 209.330246 & 52.731747 & 0.180 & -23.50 & 11.53 & 0.639 & $\mathrm{R}$ \\
\hline W3-112 & 4 & $\mathrm{~L}, \mathrm{~A}$ & 209.395874 & 52.810326 & 0.092 & -21.50 & 10.33 & 0.289 & $\mathrm{~B}$ \\
\hline W3-113 & 0 & - & 209.500320 & 52.883415 & 0.067 & -20.44 & 10.31 & 0.647 & $\mathrm{R}$ \\
\hline W3-114 & 0 & - & 209.289475 & 52.941017 & 0.073 & -20.71 & 10.07 & 0.331 & B \\
\hline W3-115 & 0 & - & 209.898544 & 52.953594 & 0.120 & -22.09 & 10.97 & 0.648 & $\mathrm{R}$ \\
\hline W3-116 & 0 & - & 209.505081 & 52.963158 & 0.119 & -21.85 & 10.85 & 0.626 & $\mathrm{R}$ \\
\hline W3-117 & 4 & $\mathrm{~A}$ & 209.858505 & 52.992821 & 0.090 & -21.70 & 10.80 & 0.634 & $\mathrm{R}$ \\
\hline W3-118 & 0 & - & 209.485703 & 53.001759 & 0.120 & -21.99 & 10.91 & 0.631 & $\mathrm{R}$ \\
\hline W3-119 & 0 & - & 209.408997 & 53.023777 & 0.120 & -22.00 & 10.92 & 0.631 & $\mathrm{R}$ \\
\hline W3-123 & 1 & - & 210.910278 & 52.205021 & 0.172 & -22.96 & 11.27 & 0.600 & $\mathrm{R}$ \\
\hline W3-125 & 0 & - & 212.192047 & 52.354225 & 0.125 & -22.18 & 10.95 & 0.596 & $\mathrm{R}$ \\
\hline W3-127 & 0 & - & 211.565323 & 52.543030 & 0.155 & -22.92 & 11.06 & 0.433 & B \\
\hline W3-132 & 0 & - & 212.096909 & 52.917885 & 0.105 & -22.32 & 10.71 & 0.335 & $\mathrm{~B}$ \\
\hline W3-133 & 0 & - & 212.649674 & 52.252735 & 0.123 & -21.97 & 10.44 & 0.212 & B \\
\hline W3-134 & 0 & - & 213.330643 & 52.311333 & 0.146 & -22.55 & 11.05 & 0.552 & $\mathrm{R}$ \\
\hline W3-135 & 0 & - & 212.903153 & 52.342899 & 0.083 & -20.97 & 10.08 & 0.246 & $\mathrm{~B}$ \\
\hline W3-136 & 0 & - & 212.533722 & 52.351055 & 0.060 & -20.26 & 10.03 & 0.460 & $\mathrm{~B}$ \\
\hline W3-138 & 0 & - & 213.503693 & 52.394409 & 0.131 & -22.12 & 10.94 & 0.611 & $\mathrm{R}$ \\
\hline W3-140 & 2 & - & 212.540070 & 52.422321 & 0.083 & -21.57 & 10.52 & 0.426 & B \\
\hline W3-142 & 0 & - & 212.626358 & 52.538002 & 0.073 & -20.83 & 10.03 & 0.254 & $\mathrm{~B}$ \\
\hline W3-143 & 0 & - & 212.727081 & 52.597843 & 0.062 & -20.55 & 10.29 & 0.591 & $\mathrm{R}$ \\
\hline W3-144 & 4 & A & 212.919144 & 52.635445 & 0.150 & -23.04 & 11.27 & 0.578 & $\mathrm{R}$ \\
\hline W3-145 & 4 & $\mathrm{~A}, \mathrm{~F}$ & 212.916229 & 52.654568 & 0.150 & -22.79 & 11.16 & 0.571 & $\mathrm{R}$ \\
\hline W3-147 & 0 & - & 212.901810 & 52.708626 & 0.090 & -21.89 & 10.83 & 0.592 & $\mathrm{R}$ \\
\hline W3-149 & 0 & - & 213.455978 & 52.786572 & 0.122 & -22.33 & 11.03 & 0.609 & $\mathrm{R}$ \\
\hline W3-150 & 0 & - & 213.598999 & 52.786713 & 0.121 & -21.85 & 10.82 & 0.601 & $\mathrm{R}$ \\
\hline W3-151 & 3 & A & 213.497894 & 52.784462 & 0.135 & -21.98 & 10.34 & 0.121 & $\mathrm{~B}$ \\
\hline W3-152 & 4 & $\mathrm{~A}$ & 212.945465 & 52.799118 & 0.104 & -21.79 & 10.79 & 0.591 & $\mathrm{R}$ \\
\hline W3-153 & 0 & - & 212.943024 & 52.861485 & 0.122 & -22.14 & 10.93 & 0.591 & $\mathrm{R}$ \\
\hline W3-155 & 0 & - & 214.632416 & 52.212444 & 0.118 & -21.80 & 10.58 & 0.397 & $\mathrm{~B}$ \\
\hline W3-156 & 0 & - & 213.941483 & 52.224419 & 0.118 & -21.90 & 10.86 & 0.616 & $\mathrm{R}$ \\
\hline W3-157 & 1 & - & 213.935242 & 52.315334 & 0.150 & -22.66 & 11.17 & 0.617 & $\mathrm{R}$ \\
\hline W3-158 & 0 & - & 213.737656 & 52.343651 & 0.083 & -20.96 & 10.47 & 0.604 & $\mathrm{R}$ \\
\hline W3-160 & 0 & - & 213.854080 & 52.424507 & 0.100 & -21.77 & 10.70 & 0.522 & $\mathrm{~B}$ \\
\hline W3-164 & 4 & $\mathrm{~A}$ & 214.529358 & 52.697182 & 0.151 & -23.06 & 11.33 & 0.623 & $\mathrm{R}$ \\
\hline W3-165 & 1 & - & 213.873688 & 52.779507 & 0.185 & -23.04 & 11.22 & 0.533 & $\mathrm{~B}$ \\
\hline W3-166 & 0 & - & 213.923721 & 52.813126 & 0.150 & -23.14 & 11.15 & 0.429 & B \\
\hline W3-169 & 0 & - & 214.629379 & 52.894325 & 0.172 & -23.71 & 11.37 & 0.430 & $\mathrm{~B}$ \\
\hline W3-171 & 1 & - & 214.626129 & 52.903076 & 0.080 & -21.55 & 10.66 & 0.564 & $\mathrm{R}$ \\
\hline
\end{tabular}


TABLE 4-Continued

\begin{tabular}{|c|c|c|c|c|c|c|c|c|c|}
\hline ID & Confidence $^{\mathrm{b}}$ & Features $^{c}$ & $\begin{array}{c}\text { RA } \\
{[\mathrm{J} 2000]}\end{array}$ & $\begin{array}{c}\text { Dec } \\
{[\mathrm{J} 2000]}\end{array}$ & $z_{\text {phot }}$ & $\begin{array}{c}\mathrm{M}_{r}^{\prime \mathrm{d}} \\
{[\mathrm{mag}]}\end{array}$ & $\begin{array}{c}\mathrm{M}_{\star}{ }^{\mathrm{e}} \\
{\left[\log \left(\mathrm{M}_{\odot}\right)\right]}\end{array}$ & $\begin{array}{c}\left(g^{\prime}-r^{\prime}\right)^{\mathrm{f}} \\
{[\mathrm{mag}]}\end{array}$ & Red/Blue ${ }^{\mathrm{g}}$ \\
\hline W3-173 & 4 & $\mathrm{~A}, \mathrm{M}$ & 214.348984 & 52.964146 & 0.151 & -23.20 & 11.41 & 0.644 & $\mathrm{R}$ \\
\hline W3-174 & 0 & - & 216.243637 & 52.493702 & 0.159 & -23.14 & 11.29 & 0.563 & $\mathrm{R}$ \\
\hline W3-175 & 2 & - & 216.063599 & 52.507214 & 0.083 & -20.91 & 10.22 & 0.395 & B \\
\hline W3-176 & 0 & - & 215.329254 & 52.542801 & 0.113 & -22.53 & 11.10 & 0.602 & $\mathrm{R}$ \\
\hline W3-177 & 0 & - & 215.482788 & 52.813068 & 0.090 & -21.27 & 10.66 & 0.660 & $\mathrm{R}$ \\
\hline W3-178 & 0 & - & 216.419113 & 52.819981 & 0.148 & -22.38 & 11.08 & 0.643 & $\mathrm{R}$ \\
\hline W3-179 & 0 & - & 215.880478 & 52.923363 & 0.083 & -20.98 & 10.20 & 0.351 & B \\
\hline W3-180 & 2 & - & 215.478272 & 53.004913 & 0.113 & -21.96 & 10.78 & 0.520 & B \\
\hline W3-181 & 0 & - & 218.209183 & 52.305428 & 0.076 & -21.10 & 10.23 & 0.338 & B \\
\hline W3-182 & 0 & - & 216.806152 & 52.399116 & 0.099 & -21.64 & 10.54 & 0.420 & B \\
\hline W3-183 & 0 & - & 217.106079 & 52.456772 & 0.129 & -22.28 & 10.99 & 0.597 & $\mathrm{R}$ \\
\hline W3-187 & 0 & - & 216.780335 & 52.833733 & 0.120 & -22.01 & 10.94 & 0.646 & $\mathrm{R}$ \\
\hline W3-188 & 3 & A & 217.734665 & 52.970123 & 0.169 & -23.45 & 11.17 & 0.339 & B \\
\hline W3-189 & 0 & - & 217.759003 & 52.985268 & 0.160 & -23.17 & 11.14 & 0.415 & B \\
\hline W3-190 & 0 & - & 218.293564 & 52.225994 & 0.082 & -21.15 & 10.32 & 0.401 & B \\
\hline W3-191 & 0 & - & 218.594971 & 52.477631 & 0.139 & -22.46 & 10.63 & 0.208 & B \\
\hline W3-192 & 0 & - & 218.660126 & 52.476520 & 0.120 & -21.89 & 10.79 & 0.554 & $\mathrm{R}$ \\
\hline W3-193 & 0 & - & 219.006836 & 52.532066 & 0.086 & -21.05 & 10.23 & 0.353 & B \\
\hline W3-194 & 0 & - & 219.133163 & 52.644855 & 0.143 & -22.76 & 10.97 & 0.405 & B \\
\hline W3-195 & 0 & - & 218.493957 & 52.657406 & 0.173 & -23.12 & 11.03 & 0.333 & B \\
\hline W3-196 & 0 & - & 218.587921 & 52.685917 & 0.164 & -22.77 & 11.00 & 0.434 & B \\
\hline W3-197 & 0 & - & 219.174988 & 52.703667 & 0.120 & -22.00 & 10.90 & 0.615 & $\mathrm{R}$ \\
\hline W3-198 & 0 & - & 218.301025 & 52.732204 & 0.060 & -20.74 & 10.37 & 0.588 & $\mathrm{R}$ \\
\hline W3-199 & 3 & $\mathrm{~S}, \mathrm{H}$ & 219.238205 & 52.761662 & 0.150 & -23.09 & 11.32 & 0.600 & $\mathrm{R}$ \\
\hline W3-200 & 0 & - & 218.724045 & 52.797436 & 0.176 & -22.94 & 11.28 & 0.617 & $\mathrm{R}$ \\
\hline W3-201 & 0 & - & 218.370041 & 52.816826 & 0.119 & -22.00 & 10.71 & 0.443 & B \\
\hline W3-202 & 0 & - & 219.062866 & 52.819733 & 0.120 & -22.13 & 10.96 & 0.622 & $\mathrm{R}$ \\
\hline W3-203 & 0 & - & 219.343277 & 52.835514 & 0.100 & -21.82 & 10.85 & 0.634 & $\mathrm{R}$ \\
\hline W3-204 & 0 & - & 219.431351 & 52.928234 & 0.182 & -23.74 & 11.61 & 0.628 & $\mathrm{R}$ \\
\hline W3-206 & 0 & - & 218.293991 & 52.975128 & 0.087 & -21.37 & 10.64 & 0.611 & $\mathrm{R}$ \\
\hline W3-207 & 1 & - & 218.282562 & 53.021771 & 0.117 & -21.98 & 10.67 & 0.416 & B \\
\hline W3-209 & 4 & $\mathrm{~S}$ & 210.362747 & 53.170868 & 0.137 & -22.34 & 10.94 & 0.527 & B \\
\hline W3-210 & 0 & - & 209.149353 & 53.217339 & 0.080 & -21.23 & 10.33 & 0.385 & B \\
\hline W3-211 & 4 & $\mathrm{M}, \mathrm{A}$ & 209.158005 & 53.236908 & 0.079 & -21.31 & 10.37 & 0.389 & B \\
\hline W3-212 & 4 & $\mathrm{M}, \mathrm{A}$ & 209.159347 & 53.238670 & 0.113 & -21.67 & 10.58 & 0.449 & B \\
\hline W3-213 & 0 & - & 209.227921 & 53.282810 & 0.126 & -21.93 & 10.76 & 0.513 & B \\
\hline W3-217 & 0 & - & 209.706406 & 53.480301 & 0.083 & -20.98 & 10.28 & 0.430 & B \\
\hline W3-218 & 0 & - & 209.378998 & 53.492874 & 0.180 & -22.97 & 11.28 & 0.606 & $\mathrm{R}$ \\
\hline W3-219 & 0 & - & 209.320465 & 53.505669 & 0.160 & -22.64 & 11.09 & 0.556 & $\mathrm{R}$ \\
\hline W3-220 & 0 & - & 209.882095 & 53.752670 & 0.110 & -22.45 & 10.89 & 0.444 & B \\
\hline W3-221 & 1 & - & 209.868240 & 53.750801 & 0.090 & -21.48 & 10.72 & 0.645 & $\mathrm{R}$ \\
\hline W3-223 & 2 & - & 209.271423 & 53.796322 & 0.096 & -22.13 & 10.87 & 0.539 & $\mathrm{R}$ \\
\hline W3-224 & 0 & - & 209.898498 & 53.857487 & 0.127 & -22.05 & 10.90 & 0.595 & $\mathrm{R}$ \\
\hline W3-225 & 0 & - & 209.862823 & 53.864845 & 0.076 & -20.85 & 10.14 & 0.351 & B \\
\hline
\end{tabular}


TABLE 4-Continued

\begin{tabular}{|c|c|c|c|c|c|c|c|c|c|}
\hline ID & Confidence $^{\mathrm{b}}$ & Features $^{c}$ & $\begin{array}{c}\mathrm{RA} \\
{[\mathrm{J} 2000]}\end{array}$ & $\begin{array}{c}\text { Dec } \\
{[\mathrm{J} 2000]}\end{array}$ & $z_{p h o t}$ & $\begin{array}{c}\mathrm{M}_{r}^{\prime \mathrm{d}} \\
{[\mathrm{mag}]}\end{array}$ & $\begin{array}{c}\mathrm{M}_{\star}^{\mathrm{e}} \\
{\left[\log \left(\mathrm{M}_{\odot}\right)\right]}\end{array}$ & $\begin{array}{c}\left(g^{\prime}-r^{\prime}\right)^{\mathrm{f}} \\
{[\mathrm{mag}]}\end{array}$ & Red/Blue ${ }^{\mathrm{g}}$ \\
\hline W3-226 & 2 & - & 209.741440 & 53.904179 & 0.154 & -23.00 & 11.26 & 0.585 & $\mathrm{R}$ \\
\hline W3-227 & 0 & - & 211.299377 & 53.115326 & 0.091 & -21.47 & 10.37 & 0.336 & $\mathrm{~B}$ \\
\hline W3-228 & 1 & - & 211.885223 & 53.144672 & 0.134 & -22.54 & 11.02 & 0.527 & $\mathrm{~B}$ \\
\hline W3-230 & 1 & - & 211.488709 & 53.511341 & 0.129 & -22.03 & 10.78 & 0.495 & B \\
\hline W3-231 & 0 & - & 211.888779 & 53.732769 & 0.145 & -22.79 & 10.94 & 0.372 & B \\
\hline W3-232 & 0 & - & 210.785950 & 53.727581 & 0.060 & -20.34 & 10.22 & 0.600 & $\mathrm{R}$ \\
\hline W3-234 & 0 & - & 210.904633 & 53.807507 & 0.124 & -22.67 & 10.89 & 0.370 & $\mathrm{~B}$ \\
\hline W3-236 & 0 & - & 212.061478 & 53.837715 & 0.145 & -22.85 & 11.04 & 0.431 & B \\
\hline W3-237 & 2 & - & 211.330795 & 53.865372 & 0.108 & -21.71 & 10.77 & 0.604 & $\mathrm{R}$ \\
\hline W3-238 & 0 & - & 211.975235 & 53.905971 & 0.060 & -20.21 & 10.15 & 0.589 & $\mathrm{R}$ \\
\hline W3-239 & 3 & $\mathrm{~A}$ & 211.729981 & 53.925156 & 0.060 & -20.31 & 10.17 & 0.568 & $\mathrm{R}$ \\
\hline W3-240 & 0 & - & 211.830338 & 53.932800 & 0.150 & -22.71 & 11.16 & 0.593 & $\mathrm{R}$ \\
\hline W3-243 & 4 & $\mathrm{~A}, \mathrm{~S}, \mathrm{M}$ & 211.850418 & 54.004223 & 0.089 & -21.25 & 10.56 & 0.576 & $\mathrm{R}$ \\
\hline W3-245 & 0 & - & 212.177994 & 53.998447 & 0.151 & -22.82 & 11.21 & 0.600 & $\mathrm{R}$ \\
\hline W3-246 & 4 & $\mathrm{~A}, \mathrm{~F}$ & 211.857315 & 54.009377 & 0.150 & -22.87 & 11.25 & 0.619 & $\mathrm{R}$ \\
\hline W3-247 & 0 & - & 212.131134 & 54.032150 & 0.156 & -22.66 & 10.91 & 0.388 & $\mathrm{~B}$ \\
\hline W3-248 & 0 & - & 212.929306 & 53.258701 & 0.154 & -22.58 & 11.14 & 0.625 & $\mathrm{R}$ \\
\hline W3-250 & 0 & - & 212.358276 & 53.416184 & 0.119 & -22.35 & 11.05 & 0.625 & $\mathrm{R}$ \\
\hline W3-251 & 0 & - & 212.503540 & 53.423107 & 0.122 & -22.10 & 10.92 & 0.598 & $\mathrm{R}$ \\
\hline W3-252 & 0 & - & 213.694290 & 53.479568 & 0.101 & -22.47 & 10.88 & 0.427 & $\mathrm{~B}$ \\
\hline W3-253 & 0 & - & 212.535065 & 53.640400 & 0.077 & -21.45 & 10.30 & 0.275 & $\mathrm{~B}$ \\
\hline W3-254 & 0 & - & 212.910584 & 53.620235 & 0.183 & -23.32 & 11.09 & 0.316 & $\mathrm{~B}$ \\
\hline W3-255 & 0 & - & 213.104614 & 53.632404 & 0.121 & -22.39 & 11.04 & 0.602 & $\mathrm{R}$ \\
\hline W3-256 & 2 & - & 212.563415 & 53.658115 & 0.131 & -22.60 & 11.14 & 0.621 & $\mathrm{R}$ \\
\hline W3-257 & 2 & - & 212.921493 & 53.651966 & 0.071 & -20.75 & 10.20 & 0.432 & $\mathrm{~B}$ \\
\hline W3-258 & 0 & - & 212.420593 & 53.655346 & 0.165 & -23.20 & 11.38 & 0.613 & $\mathrm{R}$ \\
\hline W3-259 & 0 & - & 212.570328 & 53.668453 & 0.097 & -21.50 & 10.49 & 0.429 & B \\
\hline W3-261 & 1 & - & 212.515091 & 53.763378 & 0.152 & -23.23 & 11.42 & 0.645 & $\mathrm{R}$ \\
\hline W3-262 & 0 & - & 212.271851 & 53.795422 & 0.088 & -21.20 & 10.27 & 0.338 & B \\
\hline W3-263 & 3 & A & 212.705353 & 53.772068 & 0.102 & -21.65 & 10.70 & 0.557 & $\mathrm{R}$ \\
\hline W3-264 & 1 & - & 212.880936 & 53.795136 & 0.191 & -23.25 & 11.40 & 0.615 & $\mathrm{R}$ \\
\hline W3-266 & 0 & - & 212.231049 & 53.824078 & 0.118 & -22.28 & 10.97 & 0.580 & $\mathrm{R}$ \\
\hline W3-270 & 0 & - & 213.141983 & 54.009670 & 0.174 & -23.05 & 11.29 & 0.590 & $\mathrm{R}$ \\
\hline W3-271 & 0 & - & 214.731949 & 53.108681 & 0.147 & -22.62 & 11.14 & 0.611 & $\mathrm{R}$ \\
\hline W3-272 & 4 & $\mathrm{~S}$ & 215.052277 & 53.124859 & 0.107 & -21.89 & 10.82 & 0.580 & $\mathrm{R}$ \\
\hline W3-273 & 3 & $\mathrm{~A}, \mathrm{~F}$ & 214.062256 & 53.146286 & 0.180 & -22.91 & 11.27 & 0.618 & $\mathrm{R}$ \\
\hline W3-274 & 3 & A & 214.933639 & 53.178600 & 0.120 & -22.62 & 11.14 & 0.609 & $\mathrm{R}$ \\
\hline W3-275 & 0 & - & 214.872299 & 53.194256 & 0.145 & -22.24 & 10.70 & 0.352 & B \\
\hline W3-276 & 0 & - & 214.998291 & 53.337639 & 0.112 & -21.65 & 10.61 & 0.478 & $\mathrm{~B}$ \\
\hline W3-277 & 0 & - & 214.112030 & 53.380070 & 0.120 & -22.07 & 10.94 & 0.625 & $\mathrm{R}$ \\
\hline W3-278 & 0 & - & 213.741882 & 53.588757 & 0.115 & -22.57 & 10.87 & 0.387 & $\mathrm{~B}$ \\
\hline W3-280 & 0 & - & 213.979172 & 53.587486 & 0.168 & -22.91 & 10.77 & 0.169 & B \\
\hline W3-281 & 0 & - & 214.461975 & 53.607838 & 0.170 & -23.45 & 11.24 & 0.405 & $\mathrm{~B}$ \\
\hline W3-282 & 0 & - & 215.132873 & 53.601181 & 0.180 & -22.93 & 11.27 & 0.615 & $\mathrm{R}$ \\
\hline
\end{tabular}


TABLE 4-Continued

\begin{tabular}{|c|c|c|c|c|c|c|c|c|c|}
\hline ID & Confidence $^{\mathrm{b}}$ & Features $^{c}$ & $\begin{array}{c}\text { RA } \\
{[\mathrm{J} 2000]}\end{array}$ & $\begin{array}{c}\text { Dec } \\
{[\mathrm{J} 2000]}\end{array}$ & $z_{\text {phot }}$ & $\begin{array}{c}\mathrm{M}_{r}^{\prime \mathrm{d}} \\
{[\mathrm{mag}]}\end{array}$ & $\begin{array}{c}\mathrm{M}_{\star}{ }^{\mathrm{e}} \\
{\left[\log \left(\mathrm{M}_{\odot}\right)\right]}\end{array}$ & $\begin{array}{c}\left(g^{\prime}-r^{\prime}\right)^{\mathrm{f}} \\
{[\mathrm{mag}]}\end{array}$ & Red/Blue ${ }^{\mathrm{g}}$ \\
\hline W3-283 & 4 & $\mathrm{~S}$ & 214.942932 & 53.612072 & 0.150 & -22.49 & 11.15 & 0.664 & $\mathrm{R}$ \\
\hline W3-284 & 4 & $\mathrm{~F}$ & 214.572891 & 53.788956 & 0.138 & -23.30 & 11.39 & 0.591 & $\mathrm{R}$ \\
\hline W3-285 & 0 & - & 214.511124 & 53.784096 & 0.120 & -22.18 & 11.00 & 0.638 & $\mathrm{R}$ \\
\hline W3-286 & 0 & - & 214.111115 & 53.809334 & 0.095 & -21.22 & 10.19 & 0.259 & B \\
\hline W3-287 & 1 & - & 214.078598 & 53.841564 & 0.125 & -22.12 & 10.97 & 0.636 & $\mathrm{R}$ \\
\hline W3-288 & 0 & - & 215.125763 & 53.866497 & 0.094 & -21.51 & 10.47 & 0.406 & B \\
\hline W3-292 & 0 & - & 216.622604 & 53.311359 & 0.121 & -21.98 & 10.71 & 0.451 & B \\
\hline W3-293 & 0 & - & 216.472351 & 53.327595 & 0.085 & -21.24 & 10.51 & 0.537 & $\mathrm{R}$ \\
\hline W3-294 & 4 & $\mathrm{~A}$ & 215.877731 & 53.473263 & 0.061 & -20.28 & 10.21 & 0.618 & $\mathrm{R}$ \\
\hline W3-296 & 3 & $\mathrm{~L}, \mathrm{H}$ & 216.543228 & 53.697403 & 0.181 & -22.91 & 11.27 & 0.620 & $\mathrm{R}$ \\
\hline W3-298 & 2 & - & 216.110794 & 53.738377 & 0.168 & -23.01 & 11.31 & 0.623 & $\mathrm{R}$ \\
\hline W3-299 & 1 & - & 215.489700 & 53.840237 & 0.154 & -22.56 & 11.17 & 0.652 & $\mathrm{R}$ \\
\hline W3-300 & 3 & $\mathrm{~S}, \mathrm{~A}$ & 216.182754 & 53.864155 & 0.179 & -23.63 & 11.27 & 0.360 & B \\
\hline W3-301 & 0 & - & 215.778595 & 53.948265 & 0.121 & -22.11 & 10.96 & 0.633 & $\mathrm{R}$ \\
\hline W3-303 & 0 & - & 217.032608 & 53.200806 & 0.084 & -20.98 & 10.23 & 0.382 & B \\
\hline W3-304 & 4 & $\mathrm{~F}$ & 218.016815 & 53.235176 & 0.171 & -23.28 & 11.39 & 0.594 & $\mathrm{R}$ \\
\hline W3-306 & 0 & - & 218.086365 & 53.438587 & 0.178 & -22.79 & 10.86 & 0.299 & B \\
\hline W3-308 & 1 & - & 218.187241 & 53.462620 & 0.179 & -22.87 & 11.23 & 0.601 & $\mathrm{R}$ \\
\hline W3-310 & 0 & - & 217.074982 & 53.568829 & 0.120 & -22.34 & 11.08 & 0.658 & $\mathrm{R}$ \\
\hline W3-311 & 4 & $\mathrm{~A}, \mathrm{~L}$ & 218.163193 & 53.586056 & 0.127 & -22.21 & 10.92 & 0.554 & $\mathrm{R}$ \\
\hline W3-312 & 0 & - & 218.083038 & 53.613880 & 0.150 & -22.63 & 11.21 & 0.667 & $\mathrm{R}$ \\
\hline W3-313 & 0 & - & 216.776779 & 53.621136 & 0.115 & -21.89 & 10.77 & 0.540 & $\mathrm{R}$ \\
\hline W3-314 & 0 & - & 217.552505 & 53.925854 & 0.090 & -21.44 & 10.37 & 0.345 & B \\
\hline W3-315 & 1 & - & 217.364380 & 53.929970 & 0.151 & -22.43 & 10.87 & 0.437 & B \\
\hline W3-318 & 0 & - & 218.717087 & 53.116196 & 0.095 & -21.42 & 10.36 & 0.340 & B \\
\hline W3-319 & 1 & - & 218.689728 & 53.271534 & 0.145 & -22.45 & 10.80 & 0.364 & B \\
\hline W3-320 & 4 & $\mathrm{H}$ & 219.292465 & 53.306797 & 0.150 & -23.16 & 11.33 & 0.587 & $\mathrm{R}$ \\
\hline W3-321 & 0 & - & 218.451843 & 53.321220 & 0.067 & -20.85 & 10.40 & 0.577 & $\mathrm{R}$ \\
\hline W3-323 & 0 & - & 219.340042 & 53.445568 & 0.149 & -23.11 & 11.04 & 0.346 & B \\
\hline W3-324 & 0 & - & 219.569824 & 53.435070 & 0.178 & -22.87 & 11.23 & 0.600 & $\mathrm{R}$ \\
\hline W3-325 & 0 & - & 218.626083 & 53.459709 & 0.105 & -21.95 & 10.73 & 0.487 & B \\
\hline W3-326 & 0 & - & 218.418457 & 53.454117 & 0.132 & -22.25 & 10.97 & 0.588 & $\mathrm{R}$ \\
\hline W3-327 & 1 & - & 219.546112 & 53.462536 & 0.137 & -22.26 & 10.92 & 0.538 & $\mathrm{R}$ \\
\hline W3-328 & 2 & - & 219.499527 & 53.518875 & 0.178 & -22.88 & 11.23 & 0.596 & $\mathrm{R}$ \\
\hline W3-329 & 0 & - & 219.692154 & 53.649200 & 0.049 & -20.27 & 9.84 & 0.288 & B \\
\hline W3-330 & 0 & - & 218.644486 & 53.684467 & 0.150 & -22.44 & 11.06 & 0.600 & $\mathrm{R}$ \\
\hline W3-331 & 2 & - & 219.792877 & 53.693619 & 0.119 & -22.24 & 10.98 & 0.605 & $\mathrm{R}$ \\
\hline W3-333 & 4 & $\mathrm{H}$ & 219.025299 & 53.798485 & 0.193 & -23.75 & 11.59 & 0.610 & $\mathrm{R}$ \\
\hline W3-335 & 0 & - & 218.476990 & 53.875362 & 0.122 & -21.88 & 10.59 & 0.386 & B \\
\hline W3-339 & 3 & M & 209.096008 & 54.160988 & 0.154 & -22.66 & 11.17 & 0.623 & $\mathrm{R}$ \\
\hline W3-340 & 0 & - & 209.319412 & 54.210136 & 0.054 & -20.06 & 9.58 & 0.129 & B \\
\hline W3-341 & 4 & $\mathrm{~L}$ & 209.544098 & 54.246803 & 0.060 & -20.35 & 10.22 & 0.599 & $\mathrm{R}$ \\
\hline W3-342 & 0 & - & 210.077286 & 54.480289 & 0.073 & -21.25 & 10.51 & 0.537 & $\mathrm{R}$ \\
\hline W3-343 & 0 & - & 209.986847 & 54.538315 & 0.123 & -22.08 & 10.62 & 0.338 & B \\
\hline
\end{tabular}


TABLE 4-Continued

\begin{tabular}{|c|c|c|c|c|c|c|c|c|c|}
\hline ID & Confidence $^{\mathrm{b}}$ & Features $^{c}$ & $\begin{array}{c}\mathrm{RA} \\
{[\mathrm{J} 2000]}\end{array}$ & $\begin{array}{c}\text { Dec } \\
{[\mathrm{J} 2000]}\end{array}$ & $z_{p h o t}$ & $\begin{array}{c}\mathrm{M}_{r}^{\prime \mathrm{d}} \\
{[\mathrm{mag}]}\end{array}$ & $\begin{array}{c}\mathrm{M}_{\star}^{\mathrm{e}} \\
{\left[\log \left(\mathrm{M}_{\odot}\right)\right]}\end{array}$ & $\begin{array}{c}\left(g^{\prime}-r^{\prime}\right)^{\mathrm{f}} \\
{[\mathrm{mag}]}\end{array}$ & Red/Blue ${ }^{\mathrm{g}}$ \\
\hline W3-344 & 0 & - & 209.420425 & 54.679054 & 0.082 & -21.24 & 10.53 & 0.552 & $\mathrm{R}$ \\
\hline W3-345 & 1 & - & 209.547699 & 54.732265 & 0.120 & -21.98 & 10.85 & 0.583 & $\mathrm{R}$ \\
\hline W3-346 & 0 & - & 209.532455 & 54.755295 & 0.123 & -22.40 & 10.75 & 0.341 & $\mathrm{~B}$ \\
\hline W3-348 & 2 & - & 210.008667 & 54.779526 & 0.160 & -23.40 & 11.43 & 0.590 & $\mathrm{R}$ \\
\hline W3-350 & 3 & $\mathrm{~S}$ & 210.548492 & 54.932991 & 0.100 & -21.75 & 10.50 & 0.343 & B \\
\hline W3-352 & 4 & $\mathrm{~L}, \mathrm{~F}$ & 209.275131 & 54.940247 & 0.124 & -22.17 & 10.87 & 0.531 & $\mathrm{R}$ \\
\hline W3-353 & 3 & $\mathrm{M}$ & 209.303955 & 54.944824 & 0.154 & -22.81 & 11.19 & 0.589 & $\mathrm{R}$ \\
\hline W3-357 & 0 & - & 211.995529 & 54.363045 & 0.165 & -22.66 & 10.90 & 0.376 & B \\
\hline W3-359 & 0 & - & 211.166351 & 54.387829 & 0.147 & -22.46 & 10.82 & 0.381 & $\mathrm{~B}$ \\
\hline W3-360 & 0 & - & 211.772003 & 54.405373 & 0.093 & -21.25 & 10.54 & 0.557 & $\mathrm{R}$ \\
\hline W3-361 & 0 & - & 211.230392 & 54.441349 & 0.180 & -23.08 & 11.27 & 0.561 & $\mathrm{R}$ \\
\hline W3-363 & 1 & - & 212.004944 & 54.557308 & 0.112 & -22.12 & 10.74 & 0.431 & B \\
\hline W3-364 & 0 & - & 212.115982 & 54.655930 & 0.098 & -21.67 & 10.47 & 0.347 & $\mathrm{~B}$ \\
\hline W3-365 & 0 & - & 211.977921 & 54.703484 & 0.087 & -21.50 & 10.37 & 0.324 & B \\
\hline W3-366 & 2 & - & 211.155212 & 54.793438 & 0.132 & -22.45 & 10.79 & 0.354 & B \\
\hline W3-368 & 0 & - & 211.065872 & 54.966366 & 0.120 & -21.86 & 10.79 & 0.564 & $\mathrm{R}$ \\
\hline W3-369 & 1 & - & 212.123306 & 54.964237 & 0.089 & -21.09 & 10.09 & 0.212 & B \\
\hline W3-372 & 0 & - & 212.935638 & 54.158489 & 0.090 & -21.20 & 10.53 & 0.571 & $\mathrm{R}$ \\
\hline W3-375 & 0 & - & 212.425156 & 54.236733 & 0.082 & -21.72 & 10.68 & 0.519 & B \\
\hline W3-376 & 3 & A & 213.177521 & 54.266514 & 0.150 & -23.30 & 11.35 & 0.551 & $\mathrm{R}$ \\
\hline W3-377 & 0 & - & 212.300415 & 54.333485 & 0.152 & -22.92 & 11.18 & 0.540 & $\mathrm{R}$ \\
\hline W3-378 & 0 & - & 212.395416 & 54.442329 & 0.136 & -22.39 & 10.99 & 0.554 & $\mathrm{R}$ \\
\hline W3-379 & 0 & - & 212.338608 & 54.491951 & 0.071 & -20.69 & 10.05 & 0.326 & B \\
\hline W3-380 & 0 & - & 213.609879 & 54.505718 & 0.090 & -21.18 & 10.31 & 0.385 & B \\
\hline W3-381 & 4 & M & 212.262207 & 54.551937 & 0.149 & -22.44 & 10.73 & 0.305 & $\mathrm{~B}$ \\
\hline W3-382 & 0 & - & 212.762207 & 54.531582 & 0.118 & -22.13 & 10.71 & 0.404 & $\mathrm{~B}$ \\
\hline W3-383 & 2 & - & 212.256546 & 54.555839 & 0.082 & -21.29 & 10.40 & 0.419 & B \\
\hline W3-385 & 0 & - & 212.252380 & 54.571976 & 0.090 & -21.59 & 10.69 & 0.576 & $\mathrm{R}$ \\
\hline W3-386 & 0 & - & 212.311401 & 54.581997 & 0.088 & -21.19 & 10.49 & 0.543 & $\mathrm{R}$ \\
\hline W3-387 & 0 & - & 212.784698 & 54.593464 & 0.143 & -22.56 & 11.02 & 0.526 & B \\
\hline W3-388 & 0 & - & 212.347671 & 54.599747 & 0.090 & -21.10 & 10.38 & 0.475 & $\mathrm{~B}$ \\
\hline W3-389 & 0 & - & 213.011124 & 54.650566 & 0.140 & -22.71 & 11.13 & 0.567 & $\mathrm{R}$ \\
\hline W3-390 & 0 & - & 212.774796 & 54.679138 & 0.133 & -22.31 & 10.99 & 0.583 & $\mathrm{R}$ \\
\hline W3-391 & 0 & - & 213.595871 & 54.706509 & 0.066 & -21.09 & 10.47 & 0.557 & $\mathrm{R}$ \\
\hline W3-392 & 0 & - & 213.674393 & 54.721996 & 0.088 & -21.60 & 10.60 & 0.488 & $\mathrm{~B}$ \\
\hline W3-393 & 0 & - & 213.358948 & 54.739876 & 0.097 & -21.32 & 10.58 & 0.575 & $\mathrm{R}$ \\
\hline W3-394 & 0 & - & 213.479492 & 54.763748 & 0.180 & -23.16 & 11.33 & 0.585 & $\mathrm{R}$ \\
\hline W3-395 & 0 & - & 212.226685 & 54.789204 & 0.106 & -21.99 & 10.43 & 0.199 & B \\
\hline W3-396 & 0 & - & 213.140060 & 54.769024 & 0.154 & -22.57 & 11.10 & 0.587 & $\mathrm{R}$ \\
\hline W3-397 & 0 & - & 212.205200 & 54.856037 & 0.126 & -22.24 & 10.72 & 0.364 & $\mathrm{~B}$ \\
\hline W3-398 & 0 & - & 212.789459 & 54.946629 & 0.090 & -22.15 & 10.72 & 0.404 & $\mathrm{~B}$ \\
\hline W3-400 & 0 & - & 214.815430 & 54.126843 & 0.082 & -22.05 & 10.56 & 0.296 & B \\
\hline W3-401 & 0 & - & 214.912064 & 54.152737 & 0.112 & -21.89 & 10.74 & 0.515 & $\mathrm{~B}$ \\
\hline W3-403 & 3 & $\mathrm{M}$ & 213.934860 & 54.202934 & 0.059 & -20.23 & 10.18 & 0.604 & $\mathrm{R}$ \\
\hline
\end{tabular}


TABle 4-Continued

\begin{tabular}{|c|c|c|c|c|c|c|c|c|c|}
\hline ID & Confidence $^{\mathrm{b}}$ & Features $^{c}$ & $\begin{array}{c}\text { RA } \\
{[\mathrm{J} 2000]}\end{array}$ & $\begin{array}{c}\text { Dec } \\
{[\mathrm{J} 2000]}\end{array}$ & $z_{\text {phot }}$ & $\begin{array}{c}\mathrm{M}_{r}^{\prime \mathrm{d}} \\
{[\mathrm{mag}]}\end{array}$ & $\begin{array}{c}\mathrm{M}_{\star}{ }^{\mathrm{e}} \\
{\left[\log \left(\mathrm{M}_{\odot}\right)\right]}\end{array}$ & $\begin{array}{c}\left(g^{\prime}-r^{\prime}\right)^{\mathrm{f}} \\
{[\mathrm{mag}]}\end{array}$ & Red/Blue ${ }^{\mathrm{g}}$ \\
\hline W3-404 & 0 & - & 214.475021 & 54.268238 & 0.095 & -21.61 & 10.68 & 0.557 & $\mathrm{R}$ \\
\hline W3-405 & 0 & - & 214.492859 & 54.268360 & 0.104 & -21.62 & 10.52 & 0.413 & B \\
\hline W3-406 & 0 & - & 215.145676 & 54.324493 & 0.198 & -23.42 & 11.46 & 0.614 & $\mathrm{R}$ \\
\hline W3-407 & 4 & $\mathrm{~F}, \mathrm{M}$ & 214.670135 & 54.386970 & 0.150 & -23.31 & 11.43 & 0.622 & $\mathrm{R}$ \\
\hline W3-408 & 0 & - & 215.002319 & 54.478725 & 0.094 & -22.09 & 10.72 & 0.426 & B \\
\hline W3-409 & 3 & $\mathrm{~A}$ & 214.002472 & 54.488846 & 0.119 & -22.19 & 10.74 & 0.404 & B \\
\hline W3-410 & 0 & - & 215.003433 & 54.523369 & 0.136 & -22.26 & 10.94 & 0.555 & $\mathrm{R}$ \\
\hline W3-411 & 0 & - & 214.147751 & 54.633530 & 0.086 & -22.01 & 10.63 & 0.370 & B \\
\hline W3-413 & 0 & - & 214.162903 & 54.711464 & 0.104 & -22.17 & 10.91 & 0.562 & $\mathrm{R}$ \\
\hline W3-414 & 0 & - & 215.147766 & 54.828606 & 0.149 & -22.46 & 11.06 & 0.594 & $\mathrm{R}$ \\
\hline W3-415 & 0 & - & 215.381073 & 54.057564 & 0.150 & -22.69 & 11.18 & 0.617 & $\mathrm{R}$ \\
\hline W3-416 & 0 & - & 215.481949 & 54.075027 & 0.084 & -20.96 & 10.40 & 0.542 & $\mathrm{R}$ \\
\hline W3-418 & 4 & $\mathrm{~F}$ & 215.391190 & 54.203026 & 0.180 & -22.99 & 11.29 & 0.613 & $\mathrm{R}$ \\
\hline W3-419 & 0 & - & 216.697327 & 54.229217 & 0.060 & -21.10 & 10.55 & 0.623 & $\mathrm{R}$ \\
\hline W3-421 & 0 & - & 215.504150 & 54.356323 & 0.067 & -20.44 & 10.02 & 0.388 & B \\
\hline W3-422 & 2 & - & 216.141022 & 54.418999 & 0.087 & -22.00 & 10.61 & 0.354 & B \\
\hline W3-423 & 0 & - & 215.609528 & 54.439373 & 0.090 & -21.17 & 10.45 & 0.511 & B \\
\hline W3-424 & 0 & - & 215.946182 & 54.551251 & 0.090 & -21.61 & 10.51 & 0.403 & B \\
\hline W3-425 & 0 & - & 216.312592 & 54.552280 & 0.124 & -22.60 & 11.00 & 0.495 & B \\
\hline W3-426 & 0 & - & 215.837250 & 54.730713 & 0.120 & -22.67 & 11.13 & 0.582 & $\mathrm{R}$ \\
\hline W3-427 & 3 & $\mathrm{~S}, \mathrm{~A}$ & 215.423477 & 54.828404 & 0.121 & -21.86 & 10.83 & 0.602 & $\mathrm{R}$ \\
\hline W3-428 & 1 & - & 216.496460 & 54.833511 & 0.088 & -21.08 & 10.30 & 0.405 & B \\
\hline W3-430 & 0 & - & 216.627426 & 54.938240 & 0.150 & -22.84 & 11.12 & 0.515 & B \\
\hline W3-431 & 0 & - & 216.224380 & 54.954727 & 0.093 & -21.55 & 10.72 & 0.618 & $\mathrm{R}$ \\
\hline W3-432 & 0 & - & 217.190521 & 54.078640 & 0.060 & -20.39 & 10.23 & 0.590 & $\mathrm{R}$ \\
\hline W3-433 & 0 & - & 216.957825 & 54.098724 & 0.150 & -22.49 & 11.06 & 0.583 & $\mathrm{R}$ \\
\hline W3-434 & 1 & - & 217.824753 & 54.113495 & 0.138 & -22.26 & 10.96 & 0.579 & $\mathrm{R}$ \\
\hline W3-435 & 0 & - & 216.928345 & 54.230267 & 0.090 & -21.79 & 10.57 & 0.395 & B \\
\hline W3-436 & 4 & M & 217.733276 & 54.283123 & 0.121 & -22.01 & 10.94 & 0.642 & $\mathrm{R}$ \\
\hline W3-437 & 0 & - & 217.896072 & 54.372257 & 0.097 & -21.30 & 10.33 & 0.356 & B \\
\hline W3-440 & 0 & - & 217.707581 & 54.648228 & 0.127 & -22.55 & 10.86 & 0.383 & B \\
\hline W3-441 & 0 & - & 217.188309 & 54.681698 & 0.094 & -21.42 & 10.47 & 0.439 & B \\
\hline W3-442 & 4 & $\mathrm{~F}, \mathrm{M}$ & 217.464722 & 54.686123 & 0.180 & -23.20 & 11.37 & 0.603 & $\mathrm{R}$ \\
\hline W3-443 & 0 & - & 217.323593 & 54.709038 & 0.150 & -22.73 & 11.19 & 0.614 & $\mathrm{R}$ \\
\hline W3-446 & 0 & - & 217.981400 & 54.876423 & 0.157 & -22.97 & 11.33 & 0.653 & $\mathrm{R}$ \\
\hline W3-448 & 0 & - & 219.541336 & 54.162456 & 0.111 & -21.74 & 10.53 & 0.377 & B \\
\hline W3-449 & 0 & - & 218.457718 & 54.229420 & 0.090 & -21.78 & 10.81 & 0.619 & $\mathrm{R}$ \\
\hline W3-450 & 3 & - & 219.408432 & 54.267662 & 0.180 & -23.15 & 11.35 & 0.612 & $\mathrm{R}$ \\
\hline W3-452 & 4 & $\mathrm{~L}$ & 219.715637 & 54.356796 & 0.101 & -21.48 & 10.62 & 0.553 & $\mathrm{R}$ \\
\hline W3-453 & 3 & $\mathrm{H}$ & 219.402222 & 54.405361 & 0.151 & -22.94 & 11.35 & 0.685 & $\mathrm{R}$ \\
\hline W3-454 & 0 & - & 218.614075 & 54.472122 & 0.107 & -21.67 & 10.78 & 0.631 & $\mathrm{R}$ \\
\hline W3-455 & 1 & - & 218.707260 & 54.534405 & 0.150 & -22.56 & 10.85 & 0.373 & B \\
\hline W3-456 & 0 & - & 219.263992 & 54.685173 & 0.131 & -22.18 & 10.95 & 0.596 & $\mathrm{R}$ \\
\hline W3-457 & 2 & - & 218.389389 & 54.780766 & 0.088 & -21.24 & 10.21 & 0.272 & B \\
\hline
\end{tabular}


TABle 4-Continued

\begin{tabular}{|c|c|c|c|c|c|c|c|c|c|}
\hline ID & Confidence $^{\mathrm{b}}$ & Features $^{c}$ & $\begin{array}{c}\text { RA } \\
{[\mathrm{J} 2000]}\end{array}$ & $\begin{array}{c}\text { Dec } \\
{[\mathrm{J} 2000]}\end{array}$ & $z_{\text {phot }}$ & $\begin{array}{c}\mathrm{M}_{r}^{\prime \mathrm{d}} \\
{[\mathrm{mag}]}\end{array}$ & $\begin{array}{c}\mathrm{M}_{\star}{ }^{\mathrm{e}} \\
{\left[\log \left(\mathrm{M}_{\odot}\right)\right]}\end{array}$ & $\begin{array}{c}\left(g^{\prime}-r^{\prime}\right)^{\mathrm{f}} \\
{[\mathrm{mag}]}\end{array}$ & Red/Blue ${ }^{\mathrm{g}}$ \\
\hline W3-459 & 0 & - & 219.811752 & 54.827934 & 0.086 & -21.13 & 10.21 & 0.311 & B \\
\hline W3-460 & 1 & - & 218.899323 & 54.828564 & 0.132 & -22.11 & 10.63 & 0.331 & B \\
\hline W3-461 & 0 & - & 218.506485 & 54.834026 & 0.180 & -22.88 & 11.27 & 0.627 & $\mathrm{R}$ \\
\hline W3-463 & 0 & - & 219.805649 & 54.968014 & 0.150 & -22.76 & 11.18 & 0.597 & $\mathrm{R}$ \\
\hline W3-464 & 0 & - & 210.331360 & 55.086922 & 0.150 & -22.41 & 11.10 & 0.647 & $\mathrm{R}$ \\
\hline W3-465 & 4 & $\mathrm{~A}$ & 209.117859 & 55.166359 & 0.061 & -20.70 & 10.34 & 0.584 & $\mathrm{R}$ \\
\hline W3-466 & 0 & - & 208.867874 & 55.207722 & 0.089 & -21.74 & 10.43 & 0.287 & B \\
\hline W3-467 & 0 & - & 209.150513 & 55.192390 & 0.180 & -23.49 & 11.49 & 0.609 & $\mathrm{R}$ \\
\hline W3-468 & 0 & - & 209.286758 & 55.189678 & 0.098 & -21.52 & 10.73 & 0.633 & $\mathrm{R}$ \\
\hline W3-469 & 0 & - & 209.198685 & 55.206921 & 0.104 & -22.21 & 10.68 & 0.343 & B \\
\hline W3-470 & 4 & $\mathrm{~A}, \mathrm{~F}$ & 208.849335 & 55.202705 & 0.150 & -22.45 & 11.09 & 0.624 & $\mathrm{R}$ \\
\hline W3-471 & 3 & $\mathrm{H}$ & 209.529312 & 55.283100 & 0.151 & -22.45 & 11.09 & 0.628 & $\mathrm{R}$ \\
\hline W3-472 & 4 & $\mathrm{~S}, \mathrm{M}$ & 209.949463 & 55.300571 & 0.150 & -22.72 & 11.19 & 0.618 & $\mathrm{R}$ \\
\hline W3-473 & 0 & - & 210.409256 & 55.308182 & 0.071 & -20.65 & 10.31 & 0.576 & $\mathrm{R}$ \\
\hline W3-474 & 0 & - & 209.671936 & 55.323101 & 0.150 & -22.40 & 11.04 & 0.601 & $\mathrm{R}$ \\
\hline W3-475 & 2 & - & 209.368576 & 55.355038 & 0.180 & -23.01 & 11.32 & 0.633 & $\mathrm{R}$ \\
\hline W3-476 & 0 & - & 209.914551 & 55.443405 & 0.099 & -21.79 & 10.78 & 0.584 & $\mathrm{R}$ \\
\hline W3-477 & 0 & - & 209.967835 & 55.465286 & 0.074 & -21.07 & 10.15 & 0.280 & B \\
\hline W3-478 & 0 & - & 209.539413 & 55.540852 & 0.123 & -21.92 & 10.86 & 0.609 & $\mathrm{R}$ \\
\hline W3-479 & 0 & - & 210.242447 & 55.568100 & 0.097 & -22.35 & 10.75 & 0.358 & B \\
\hline W3-480 & 0 & - & 210.228546 & 55.619640 & 0.150 & -22.78 & 11.24 & 0.643 & $\mathrm{R}$ \\
\hline W3-481 & 3 & $\mathrm{~F}, \mathrm{~S}$ & 209.144775 & 55.667687 & 0.161 & -22.67 & 11.15 & 0.598 & $\mathrm{R}$ \\
\hline W3-482 & 4 & $\mathrm{~A}$ & 208.942078 & 55.675217 & 0.137 & -22.86 & 11.25 & 0.620 & $\mathrm{R}$ \\
\hline W3-483 & 1 & - & 209.868133 & 55.707054 & 0.155 & -22.31 & 10.49 & 0.135 & B \\
\hline W3-484 & 0 & - & 210.153564 & 55.725655 & 0.100 & -21.80 & 10.44 & 0.276 & B \\
\hline W3-485 & 0 & - & 208.981293 & 55.760666 & 0.150 & -22.85 & 11.29 & 0.663 & $\mathrm{R}$ \\
\hline W3-486 & 0 & - & 209.032471 & 55.815609 & 0.139 & -22.63 & 10.82 & 0.318 & B \\
\hline W3-487 & 0 & - & 210.151413 & 55.871018 & 0.177 & -22.99 & 11.22 & 0.547 & $\mathrm{R}$ \\
\hline W3-488 & 0 & - & 209.957382 & 55.894707 & 0.080 & -21.14 & 10.27 & 0.361 & B \\
\hline W3-489 & 0 & - & 210.657990 & 55.023800 & 0.126 & -22.11 & 10.87 & 0.544 & $\mathrm{R}$ \\
\hline W3-491 & 1 & - & 211.729599 & 55.213062 & 0.099 & -21.48 & 10.66 & 0.587 & $\mathrm{R}$ \\
\hline W3-493 & 3 & $\mathrm{~S}, \mathrm{~L}$ & 212.003998 & 55.258217 & 0.150 & -22.43 & 11.05 & 0.597 & $\mathrm{R}$ \\
\hline W3-494 & 0 & - & 211.914780 & 55.233120 & 0.063 & -20.35 & 10.22 & 0.597 & $\mathrm{R}$ \\
\hline W3-495 & 0 & - & 211.885681 & 55.239986 & 0.150 & -22.60 & 11.16 & 0.634 & $\mathrm{R}$ \\
\hline W3-496 & 4 & $\mathrm{~S}$ & 211.766403 & 55.260757 & 0.119 & -22.26 & 11.01 & 0.624 & $\mathrm{R}$ \\
\hline W3-497 & 0 & - & 210.710159 & 55.411366 & 0.075 & -20.87 & 10.04 & 0.250 & B \\
\hline W3-499 & 0 & - & 211.756836 & 55.519615 & 0.141 & -22.58 & 10.95 & 0.454 & B \\
\hline W3-500 & 0 & - & 211.220612 & 55.677914 & 0.070 & -21.20 & 10.23 & 0.298 & B \\
\hline W3-501 & 0 & - & 210.644180 & 55.676636 & 0.180 & -22.98 & 11.34 & 0.655 & $\mathrm{R}$ \\
\hline W3-502 & 0 & - & 210.766708 & 55.708042 & 0.151 & -23.30 & 11.21 & 0.428 & B \\
\hline W3-503 & 0 & - & 211.279007 & 55.700840 & 0.129 & -22.05 & 10.70 & 0.418 & B \\
\hline W3-504 & 0 & - & 212.457245 & 55.016281 & 0.099 & -21.38 & 10.45 & 0.430 & B \\
\hline W3-505 & 1 & - & 213.535523 & 55.010467 & 0.180 & -23.40 & 11.46 & 0.618 & $\mathrm{R}$ \\
\hline W3-506 & 0 & - & 213.651459 & 55.062466 & 0.144 & -22.70 & 11.18 & 0.617 & $\mathrm{R}$ \\
\hline
\end{tabular}


TABLE 4-Continued

\begin{tabular}{|c|c|c|c|c|c|c|c|c|c|}
\hline ID & Confidence $^{\mathrm{b}}$ & Features $^{c}$ & $\begin{array}{c}\mathrm{RA} \\
{[\mathrm{J} 2000]}\end{array}$ & $\begin{array}{c}\text { Dec } \\
{[\mathrm{J} 2000]}\end{array}$ & $z_{p h o t}$ & $\begin{array}{c}\mathrm{M}_{r}^{\prime \mathrm{d}} \\
{[\mathrm{mag}]}\end{array}$ & $\begin{array}{c}\mathrm{M}_{\star}^{\mathrm{e}} \\
{\left[\log \left(\mathrm{M}_{\odot}\right)\right]}\end{array}$ & $\begin{array}{c}\left(g^{\prime}-r^{\prime}\right)^{\mathrm{f}} \\
{[\mathrm{mag}]}\end{array}$ & Red/Blue ${ }^{\mathrm{g}}$ \\
\hline W3-507 & 4 & $\mathrm{H}$ & 212.971497 & 55.109039 & 0.170 & -23.30 & 11.24 & 0.454 & $\mathrm{~B}$ \\
\hline W3-508 & 4 & $\mathrm{~A}$ & 212.272369 & 55.151615 & 0.179 & -23.23 & 11.41 & 0.629 & $\mathrm{R}$ \\
\hline W3-510 & 0 & - & 212.636581 & 55.182625 & 0.143 & -22.47 & 10.60 & 0.181 & $\mathrm{~B}$ \\
\hline W3-511 & 0 & - & 213.037079 & 55.228241 & 0.087 & -21.59 & 10.46 & 0.372 & B \\
\hline W3-515 & 2 & - & 212.177032 & 55.240601 & 0.161 & -22.72 & 11.23 & 0.651 & $\mathrm{R}$ \\
\hline W3-516 & 0 & - & 212.103363 & 55.336563 & 0.179 & -22.92 & 11.27 & 0.623 & $\mathrm{R}$ \\
\hline W3-518 & 2 & - & 212.773422 & 55.386803 & 0.092 & -21.25 & 10.31 & 0.356 & $\mathrm{~B}$ \\
\hline W3-520 & 0 & - & 212.164017 & 55.466053 & 0.098 & -21.66 & 10.80 & 0.646 & $\mathrm{R}$ \\
\hline W3-521 & 0 & - & 212.243622 & 55.495552 & 0.120 & -22.05 & 10.94 & 0.632 & $\mathrm{R}$ \\
\hline W3-522 & 0 & - & 213.572845 & 55.513000 & 0.102 & -21.85 & 10.74 & 0.532 & $\mathrm{R}$ \\
\hline W3-525 & 0 & - & 212.165024 & 55.578022 & 0.120 & -21.90 & 10.88 & 0.633 & $\mathrm{R}$ \\
\hline W3-526 & 0 & - & 212.204559 & 55.637169 & 0.145 & -22.66 & 11.15 & 0.602 & $\mathrm{R}$ \\
\hline W3-527 & 0 & - & 212.408783 & 55.647339 & 0.120 & -22.24 & 11.01 & 0.629 & $\mathrm{R}$ \\
\hline W3-528 & 0 & - & 213.480987 & 55.719357 & 0.134 & -22.15 & 10.81 & 0.483 & B \\
\hline W3-529 & 1 & - & 212.920792 & 55.796898 & 0.150 & -22.43 & 11.10 & 0.638 & $\mathrm{R}$ \\
\hline W3-531 & 0 & - & 214.848144 & 55.011856 & 0.197 & -23.18 & 11.38 & 0.620 & $\mathrm{R}$ \\
\hline W3-532 & 3 & $\mathrm{~A}$ & 214.639572 & 55.147488 & 0.164 & -23.18 & 10.86 & 0.157 & B \\
\hline W3-533 & 0 & - & 215.250748 & 55.175194 & 0.077 & -20.87 & 10.43 & 0.603 & $\mathrm{R}$ \\
\hline W3-534 & 4 & $\mathrm{~A}, \mathrm{~F}$ & 214.332459 & 55.219215 & 0.150 & -22.44 & 11.11 & 0.643 & $\mathrm{R}$ \\
\hline W3-535 & 1 & - & 214.000320 & 55.364109 & 0.094 & -21.87 & 10.75 & 0.526 & $\mathrm{R}$ \\
\hline W3-536 & 0 & - & 214.619461 & 55.457100 & 0.090 & -21.31 & 10.63 & 0.620 & $\mathrm{R}$ \\
\hline W3-537 & 0 & - & 214.895447 & 55.458962 & 0.150 & -22.63 & 11.17 & 0.636 & $\mathrm{R}$ \\
\hline W3-538 & 0 & - & 214.146668 & 55.481510 & 0.149 & -23.04 & 11.07 & 0.396 & B \\
\hline W3-539 & 0 & - & 214.176209 & 55.515049 & 0.186 & -23.45 & 11.51 & 0.642 & $\mathrm{R}$ \\
\hline W3-540 & 1 & - & 213.738373 & 55.633755 & 0.180 & -23.35 & 11.46 & 0.631 & $\mathrm{R}$ \\
\hline W3-541 & 0 & - & 214.241287 & 55.666870 & 0.146 & -22.80 & 11.05 & 0.467 & $\mathrm{~B}$ \\
\hline W3-542 & 4 & $\mathrm{~S}$ & 214.520798 & 55.678165 & 0.064 & -20.65 & 10.38 & 0.628 & $\mathrm{R}$ \\
\hline W3-544 & 0 & - & 215.049591 & 55.774597 & 0.073 & -20.69 & 10.04 & 0.318 & B \\
\hline W3-546 & 2 & - & 215.381638 & 55.046955 & 0.129 & -21.96 & 10.67 & 0.428 & B \\
\hline W3-547 & 0 & - & 216.285950 & 55.138115 & 0.109 & -21.62 & 10.60 & 0.485 & $\mathrm{~B}$ \\
\hline W3-548 & 0 & - & 216.575806 & 55.157219 & 0.078 & -21.06 & 10.20 & 0.326 & $\mathrm{~B}$ \\
\hline W3-549 & 0 & - & 215.944000 & 55.206379 & 0.074 & -20.84 & 10.16 & 0.373 & $\mathrm{~B}$ \\
\hline W3-550 & 0 & - & 216.789215 & 55.233643 & 0.106 & -21.56 & 10.73 & 0.624 & $\mathrm{R}$ \\
\hline W3-551 & 0 & - & 216.543793 & 55.242374 & 0.179 & -22.99 & 11.28 & 0.601 & $\mathrm{R}$ \\
\hline W3-552 & 3 & $\mathrm{~F}$ & 216.599075 & 55.301342 & 0.150 & -23.06 & 11.38 & 0.668 & $\mathrm{R}$ \\
\hline W3-553 & 0 & - & 216.530151 & 55.328838 & 0.150 & -22.56 & 11.18 & 0.663 & $\mathrm{R}$ \\
\hline W3-554 & 0 & - & 216.564621 & 55.329418 & 0.166 & -22.87 & 11.26 & 0.629 & $\mathrm{R}$ \\
\hline W3-555 & 0 & - & 215.411820 & 55.340649 & 0.153 & -22.68 & 11.20 & 0.641 & $\mathrm{R}$ \\
\hline W3-557 & 0 & - & 216.597214 & 55.475098 & 0.171 & -22.74 & 11.14 & 0.570 & $\mathrm{R}$ \\
\hline W3-558 & 0 & - & 216.173843 & 55.503174 & 0.116 & -21.73 & 10.65 & 0.489 & $\mathrm{~B}$ \\
\hline W3-559 & 0 & - & 216.666977 & 55.488030 & 0.180 & -23.29 & 11.49 & 0.680 & $\mathrm{R}$ \\
\hline W3-560 & 0 & - & 215.668274 & 55.547287 & 0.096 & -21.51 & 10.46 & 0.398 & B \\
\hline W3-562 & 4 & $\mathrm{~A}$ & 215.971954 & 55.667068 & 0.144 & -22.46 & 10.82 & 0.383 & $\mathrm{~B}$ \\
\hline W3-567 & 0 & - & 216.088120 & 55.742367 & 0.156 & -22.64 & 10.99 & 0.464 & $\mathrm{~B}$ \\
\hline
\end{tabular}


TABLE 4-Continued

\begin{tabular}{|c|c|c|c|c|c|c|c|c|c|}
\hline ID & Confidence $^{\mathrm{b}}$ & Features $^{c}$ & $\begin{array}{c}\mathrm{RA} \\
{[\mathrm{J} 2000]}\end{array}$ & $\begin{array}{c}\text { Dec } \\
{[\mathrm{J} 2000]}\end{array}$ & $z_{p h o t}$ & $\begin{array}{c}\mathrm{M}_{r}^{\prime \mathrm{d}} \\
{[\mathrm{mag}]}\end{array}$ & $\begin{array}{c}\mathrm{M}_{\star}^{\mathrm{e}} \\
{\left[\log \left(\mathrm{M}_{\odot}\right)\right]}\end{array}$ & $\begin{array}{c}\left(g^{\prime}-r^{\prime}\right)^{\mathrm{f}} \\
{[\mathrm{mag}]}\end{array}$ & Red/Blue ${ }^{\mathrm{g}}$ \\
\hline W3-568 & 1 & - & 215.316116 & 55.757687 & 0.153 & -22.72 & 11.23 & 0.657 & $\mathrm{R}$ \\
\hline W3-570 & 0 & - & 215.538773 & 55.783234 & 0.097 & -21.42 & 10.62 & 0.576 & $\mathrm{R}$ \\
\hline W3-571 & 0 & - & 216.254273 & 55.781845 & 0.150 & -22.58 & 11.17 & 0.648 & $\mathrm{R}$ \\
\hline W3-572 & 0 & - & 215.459900 & 55.787472 & 0.180 & -23.06 & 11.34 & 0.634 & $\mathrm{R}$ \\
\hline W3-573 & 3 & $\mathrm{~L}$ & 216.859817 & 55.819546 & 0.150 & -22.76 & 11.28 & 0.681 & $\mathrm{R}$ \\
\hline W3-574 & 2 & - & 218.069809 & 55.006325 & 0.093 & -21.69 & 10.77 & 0.607 & $\mathrm{R}$ \\
\hline W3-575 & 0 & - & 217.332565 & 55.111221 & 0.122 & -21.90 & 10.66 & 0.436 & $\mathrm{~B}$ \\
\hline W3-576 & 0 & - & 217.144577 & 55.096451 & 0.061 & -20.41 & 10.25 & 0.606 & $\mathrm{R}$ \\
\hline W3-577 & 0 & - & 218.196899 & 55.141067 & 0.102 & -21.76 & 10.72 & 0.542 & $\mathrm{R}$ \\
\hline W3-578 & 2 & - & 217.719040 & 55.205998 & 0.065 & -20.82 & 10.41 & 0.602 & $\mathrm{R}$ \\
\hline W3-579 & 0 & - & 217.268326 & 55.203339 & 0.169 & -22.71 & 11.07 & 0.511 & $\mathrm{~B}$ \\
\hline W3-580 & 2 & - & 216.971985 & 55.221546 & 0.180 & -22.88 & 11.25 & 0.613 & $\mathrm{R}$ \\
\hline W3-581 & 4 & $\mathrm{~F}$ & 217.708466 & 55.263634 & 0.110 & -22.33 & 11.02 & 0.608 & $\mathrm{R}$ \\
\hline W3-582 & 0 & - & 217.088425 & 55.367073 & 0.122 & -22.16 & 10.76 & 0.435 & B \\
\hline W3-583 & 0 & - & 217.400818 & 55.401341 & 0.136 & -22.25 & 10.98 & 0.599 & $\mathrm{R}$ \\
\hline W3-584 & 2 & - & 217.857559 & 55.443459 & 0.123 & -21.90 & 10.88 & 0.630 & $\mathrm{R}$ \\
\hline W3-585 & 0 & - & 217.224640 & 55.453125 & 0.149 & -22.61 & 11.14 & 0.609 & $\mathrm{R}$ \\
\hline W3-586 & 4 & M & 218.006836 & 55.493298 & 0.180 & -23.22 & 11.42 & 0.642 & $\mathrm{R}$ \\
\hline W3-587 & 4 & $\mathrm{~S}, \mathrm{~A}$ & 217.360260 & 55.495979 & 0.180 & -22.93 & 11.04 & 0.407 & B \\
\hline W3-589 & 0 & - & 218.290146 & 55.541634 & 0.121 & -22.44 & 11.10 & 0.637 & $\mathrm{R}$ \\
\hline W3-590 & 1 & - & 218.291901 & 55.587231 & 0.148 & -22.54 & 10.88 & 0.407 & $\mathrm{~B}$ \\
\hline W3-592 & 0 & - & 216.948181 & 55.633583 & 0.120 & -22.04 & 10.91 & 0.612 & $\mathrm{R}$ \\
\hline W3-593 & 4 & S & 217.084778 & 55.665440 & 0.056 & -20.65 & 10.32 & 0.579 & $\mathrm{R}$ \\
\hline W3-594 & 0 & - & 217.454666 & 55.720551 & 0.152 & -22.99 & 11.29 & 0.608 & $\mathrm{R}$ \\
\hline W3-595 & 0 & - & 217.469406 & 55.769699 & 0.089 & -21.06 & 10.08 & 0.221 & $\mathrm{~B}$ \\
\hline W3-596 & 0 & - & 216.872879 & 55.773930 & 0.157 & -22.53 & 11.14 & 0.641 & $\mathrm{R}$ \\
\hline W3-597 & 0 & - & 216.878876 & 55.794086 & 0.123 & -22.15 & 10.96 & 0.620 & $\mathrm{R}$ \\
\hline W3-599 & 0 & - & 216.939438 & 55.877148 & 0.180 & -23.26 & 11.41 & 0.619 & $\mathrm{R}$ \\
\hline W3-600 & 0 & - & 218.794540 & 55.063026 & 0.180 & -23.82 & 11.63 & 0.617 & $\mathrm{R}$ \\
\hline W3-601 & 0 & - & 218.540436 & 55.071095 & 0.119 & -22.17 & 10.93 & 0.583 & $\mathrm{R}$ \\
\hline W3-602 & 1 & - & 219.232437 & 55.133591 & 0.150 & -23.15 & 11.39 & 0.642 & $\mathrm{R}$ \\
\hline W3-603 & 4 & $\mathrm{~L}$ & 218.910400 & 55.135887 & 0.167 & -22.83 & 11.22 & 0.606 & $\mathrm{R}$ \\
\hline W3-604 & 0 & - & 218.912674 & 55.177967 & 0.180 & -23.18 & 11.37 & 0.613 & $\mathrm{R}$ \\
\hline W3-605 & 2 & - & 218.760300 & 55.196377 & 0.180 & -23.08 & 11.33 & 0.615 & $\mathrm{R}$ \\
\hline W3-606 & 4 & $\mathrm{~A}$ & 219.974533 & 55.275673 & 0.178 & -23.04 & 11.07 & 0.395 & $\mathrm{~B}$ \\
\hline W3-607 & 0 & - & 219.907990 & 55.443043 & 0.121 & -22.08 & 10.71 & 0.417 & B \\
\hline W3-608 & 1 & - & 218.969315 & 55.527519 & 0.123 & -22.00 & 10.95 & 0.662 & $\mathrm{R}$ \\
\hline W3-609 & 2 & - & 219.791962 & 55.544693 & 0.120 & -22.32 & 11.04 & 0.622 & $\mathrm{R}$ \\
\hline W3-610 & 0 & - & 219.654495 & 55.535358 & 0.144 & -22.49 & 10.82 & 0.368 & $\mathrm{~B}$ \\
\hline W3-611 & 1 & - & 218.969696 & 55.545967 & 0.180 & -22.92 & 11.27 & 0.616 & $\mathrm{R}$ \\
\hline W3-612 & 0 & - & 218.528275 & 55.616730 & 0.072 & -20.80 & 10.08 & 0.310 & $\mathrm{~B}$ \\
\hline W3-613 & 0 & - & 219.805328 & 55.614353 & 0.150 & -22.42 & 11.09 & 0.639 & $\mathrm{R}$ \\
\hline W3-614 & 0 & - & 218.979950 & 55.665669 & 0.081 & -21.59 & 10.44 & 0.354 & $\mathrm{~B}$ \\
\hline W3-615 & 1 & - & 219.819595 & 55.650024 & 0.067 & -20.87 & 10.45 & 0.617 & $\mathrm{R}$ \\
\hline
\end{tabular}


TABLE 4-Continued

\begin{tabular}{|c|c|c|c|c|c|c|c|c|c|}
\hline ID & Confidence $^{\mathrm{b}}$ & Features $^{c}$ & $\begin{array}{c}\text { RA } \\
{[\mathrm{J} 2000]}\end{array}$ & $\begin{array}{c}\text { Dec } \\
{[\mathrm{J} 2000]}\end{array}$ & $z_{\text {phot }}$ & $\begin{array}{c}\mathrm{M}_{r}^{\prime \mathrm{d}} \\
{[\mathrm{mag}]}\end{array}$ & $\begin{array}{c}\mathrm{M}_{\star}{ }^{\mathrm{e}} \\
{\left[\log \left(\mathrm{M}_{\odot}\right)\right]}\end{array}$ & $\begin{array}{c}\left(g^{\prime}-r^{\prime}\right)^{\mathrm{f}} \\
{[\mathrm{mag}]}\end{array}$ & Red/Blue ${ }^{\mathrm{g}}$ \\
\hline W3-619 & 0 & - & 219.340424 & 55.889736 & 0.147 & -22.56 & 10.96 & 0.467 & B \\
\hline W3-620 & 0 & - & 210.353577 & 55.911888 & 0.190 & -23.46 & 11.01 & 0.186 & $\mathrm{~B}$ \\
\hline W3-621 & 0 & - & 210.232269 & 55.932606 & 0.150 & -22.54 & 11.12 & 0.618 & $\mathrm{R}$ \\
\hline W3-623 & 0 & - & 209.687424 & 56.022244 & 0.103 & -21.49 & 10.47 & 0.420 & B \\
\hline W3-625 & 0 & - & 209.836807 & 56.186970 & 0.133 & -22.29 & 10.85 & 0.467 & B \\
\hline W3-626 & 3 & $\mathrm{~A}, \mathrm{~L}$ & 209.451737 & 56.228241 & 0.095 & -21.52 & 10.68 & 0.590 & $\mathrm{R}$ \\
\hline W3-627 & 1 & - & 209.696625 & 56.284565 & 0.120 & -22.16 & 10.99 & 0.636 & $\mathrm{R}$ \\
\hline W3-629 & 0 & - & 210.268295 & 56.421101 & 0.117 & -22.12 & 10.60 & 0.305 & B \\
\hline W3-630 & 0 & - & 209.432343 & 56.459686 & 0.158 & -22.76 & 11.22 & 0.628 & $\mathrm{R}$ \\
\hline W3-631 & 0 & - & 209.550644 & 56.482079 & 0.162 & -22.83 & 11.25 & 0.633 & $\mathrm{R}$ \\
\hline W3-632 & 0 & - & 208.925186 & 56.502041 & 0.159 & -22.79 & 11.18 & 0.583 & $\mathrm{R}$ \\
\hline W3-633 & 4 & $\mathrm{~F}$ & 208.876404 & 56.619949 & 0.180 & -23.39 & 11.49 & 0.642 & $\mathrm{R}$ \\
\hline W3-634 & 0 & - & 210.223602 & 56.753967 & 0.171 & -23.06 & 11.11 & 0.428 & B \\
\hline W3-636 & 1 & - & 210.215408 & 56.839241 & 0.119 & -21.99 & 10.90 & 0.615 & $\mathrm{R}$ \\
\hline W3-637 & 0 & - & 211.532867 & 55.968048 & 0.118 & -21.72 & 10.43 & 0.297 & B \\
\hline W3-639 & 0 & - & 211.556915 & 56.020981 & 0.127 & -22.13 & 10.53 & 0.240 & B \\
\hline W3-640 & 0 & - & 211.628494 & 56.045727 & 0.121 & -21.99 & 10.50 & 0.260 & B \\
\hline W3-641 & 0 & - & 211.614914 & 56.141468 & 0.120 & -22.57 & 11.13 & 0.613 & $\mathrm{R}$ \\
\hline W3-643 & 0 & - & 211.274994 & 56.129696 & 0.164 & -23.04 & 11.12 & 0.444 & B \\
\hline W3-644 & 0 & - & 211.259674 & 56.126534 & 0.081 & -20.89 & 10.11 & 0.304 & B \\
\hline W3-645 & 1 & - & 211.644089 & 56.132023 & 0.180 & -23.58 & 11.51 & 0.600 & $\mathrm{R}$ \\
\hline W3-646 & 0 & - & 211.111725 & 56.174515 & 0.125 & -21.95 & 10.82 & 0.566 & $\mathrm{R}$ \\
\hline W3-647 & 1 & - & 211.592117 & 56.183678 & 0.093 & -21.90 & 10.77 & 0.539 & $\mathrm{R}$ \\
\hline W3-648 & 0 & - & 211.492737 & 56.218418 & 0.079 & -21.28 & 10.44 & 0.461 & B \\
\hline W3-649 & 0 & - & 210.889450 & 56.217407 & 0.155 & -22.64 & 10.78 & 0.277 & B \\
\hline W3-651 & 0 & - & 211.071655 & 56.348755 & 0.112 & -21.86 & 10.60 & 0.398 & B \\
\hline W3-653 & 1 & - & 211.856720 & 56.379898 & 0.187 & -23.31 & 11.41 & 0.605 & $\mathrm{R}$ \\
\hline W3-654 & 2 & - & 210.581238 & 56.459492 & 0.147 & -22.66 & 11.01 & 0.479 & B \\
\hline W3-655 & 0 & - & 211.804489 & 56.486240 & 0.073 & -21.12 & 10.28 & 0.378 & B \\
\hline W3-656 & 0 & - & 211.183487 & 56.571766 & 0.090 & -21.60 & 10.49 & 0.388 & B \\
\hline W3-659 & 1 & - & 212.974289 & 56.031830 & 0.170 & -22.77 & 11.10 & 0.519 & B \\
\hline W3-660 & 4 & $\mathrm{~S}$ & 212.232697 & 56.039803 & 0.082 & -20.94 & 10.43 & 0.570 & $\mathrm{R}$ \\
\hline W3-662 & 1 & - & 212.344940 & 56.276474 & 0.120 & -22.28 & 11.04 & 0.640 & $\mathrm{R}$ \\
\hline W3-663 & 0 & - & 212.425522 & 56.449921 & 0.098 & -21.77 & 10.54 & 0.372 & B \\
\hline W3-664 & 0 & - & 212.425125 & 56.542023 & 0.164 & -22.87 & 11.05 & 0.434 & B \\
\hline W3-665 & 0 & - & 213.281692 & 56.532928 & 0.150 & -22.49 & 11.11 & 0.629 & $\mathrm{R}$ \\
\hline W3-666 & 0 & - & 212.313614 & 56.572281 & 0.122 & -22.07 & 10.89 & 0.583 & $\mathrm{R}$ \\
\hline W3-668 & 0 & - & 213.079651 & 56.808018 & 0.075 & -21.28 & 10.28 & 0.320 & B \\
\hline W3-669 & 4 & $\mathrm{~S}, \mathrm{~L}$ & 212.828232 & 56.818035 & 0.138 & -22.47 & 11.10 & 0.623 & $\mathrm{R}$ \\
\hline W3-670 & 0 & - & 213.028046 & 56.835709 & 0.160 & -23.00 & 11.22 & 0.541 & $\mathrm{R}$ \\
\hline W3-672 & 0 & - & 214.699539 & 55.928799 & 0.151 & -22.72 & 11.21 & 0.635 & $\mathrm{R}$ \\
\hline W3-673 & 0 & - & 214.660584 & 55.919517 & 0.155 & -22.49 & 11.11 & 0.628 & $\mathrm{R}$ \\
\hline W3-674 & 0 & - & 214.081650 & 55.962498 & 0.152 & -22.55 & 11.12 & 0.621 & $\mathrm{R}$ \\
\hline W3-675 & 0 & - & 214.004364 & 56.003899 & 0.087 & -21.53 & 10.48 & 0.412 & B \\
\hline
\end{tabular}


TABLE 4-Continued

\begin{tabular}{|c|c|c|c|c|c|c|c|c|c|}
\hline ID & Confidence $^{\mathrm{b}}$ & Features $^{c}$ & $\begin{array}{c}\text { RA } \\
{[\mathrm{J} 2000]}\end{array}$ & $\begin{array}{c}\text { Dec } \\
{[\mathrm{J} 2000]}\end{array}$ & $z_{\text {phot }}$ & $\begin{array}{c}\mathrm{M}_{r}^{\prime \mathrm{d}} \\
{[\mathrm{mag}]}\end{array}$ & $\begin{array}{c}\mathrm{M}_{\star}{ }^{\mathrm{e}} \\
{\left[\log \left(\mathrm{M}_{\odot}\right)\right]}\end{array}$ & $\begin{array}{c}\left(g^{\prime}-r^{\prime}\right)^{\mathrm{f}} \\
{[\mathrm{mag}]}\end{array}$ & Red/Blue ${ }^{\mathrm{g}}$ \\
\hline W3-676 & 0 & - & 214.658081 & 56.053642 & 0.120 & -22.40 & 11.10 & 0.647 & $\mathrm{R}$ \\
\hline W3-677 & 0 & - & 214.835632 & 56.102737 & 0.137 & -23.13 & 11.15 & 0.440 & B \\
\hline W3-678 & 0 & - & 214.889145 & 56.130386 & 0.085 & -21.84 & 10.68 & 0.473 & B \\
\hline W3-679 & 0 & - & 214.047745 & 56.141998 & 0.131 & -22.17 & 10.88 & 0.537 & $\mathrm{R}$ \\
\hline W3-680 & 0 & - & 213.751419 & 56.177952 & 0.087 & -21.33 & 10.45 & 0.451 & B \\
\hline W3-682 & 0 & - & 214.888840 & 56.290112 & 0.148 & -22.73 & 11.15 & 0.575 & $\mathrm{R}$ \\
\hline W3-683 & 0 & - & 214.285644 & 56.310581 & 0.152 & -22.66 & 11.17 & 0.620 & $\mathrm{R}$ \\
\hline W3-684 & 3 & $\mathrm{~A}$ & 215.268509 & 56.359219 & 0.118 & -21.93 & 10.86 & 0.603 & $\mathrm{R}$ \\
\hline W3-685 & 0 & - & 214.988236 & 56.391193 & 0.093 & -21.85 & 10.85 & 0.622 & $\mathrm{R}$ \\
\hline W3-686 & 0 & - & 214.296493 & 56.395828 & 0.139 & -22.52 & 11.02 & 0.538 & $\mathrm{R}$ \\
\hline W3-688 & 0 & - & 214.588898 & 56.482220 & 0.118 & -22.15 & 10.91 & 0.567 & $\mathrm{R}$ \\
\hline W3-689 & 1 & - & 215.253418 & 56.510746 & 0.090 & -21.75 & 10.70 & 0.525 & $\mathrm{R}$ \\
\hline W3-690 & 0 & - & 214.754120 & 56.515423 & 0.085 & -21.00 & 10.40 & 0.530 & $\mathrm{R}$ \\
\hline W3-691 & 4 & $\mathrm{~A}, \mathrm{M}$ & 214.466537 & 56.554977 & 0.071 & -21.44 & 10.63 & 0.574 & $\mathrm{R}$ \\
\hline W3-692 & 3 & $\mathrm{~A}$ & 215.130005 & 56.581638 & 0.067 & -20.81 & 10.29 & 0.497 & B \\
\hline W3-693 & 3 & $\mathrm{~A}$ & 215.065140 & 56.601692 & 0.124 & -22.02 & 10.84 & 0.552 & $\mathrm{R}$ \\
\hline W3-694 & 0 & - & 214.598724 & 56.617912 & 0.053 & -19.94 & 9.96 & 0.512 & $\mathrm{R}$ \\
\hline W3-695 & 0 & - & 214.298523 & 56.662456 & 0.102 & -21.59 & 10.68 & 0.569 & $\mathrm{R}$ \\
\hline W3-696 & 0 & - & 215.227264 & 56.814789 & 0.139 & -22.63 & 10.91 & 0.402 & B \\
\hline W3-698 & 1 & - & 216.109787 & 55.960014 & 0.113 & -21.83 & 10.78 & 0.571 & $\mathrm{R}$ \\
\hline W3-699 & 4 & $\mathrm{H}$ & 216.529816 & 55.994091 & 0.120 & -22.12 & 10.95 & 0.618 & $\mathrm{R}$ \\
\hline W3-700 & 0 & - & 215.810791 & 56.049374 & 0.090 & -21.63 & 10.58 & 0.459 & B \\
\hline W3-701 & 1 & - & 215.558472 & 56.164738 & 0.180 & -23.16 & 11.37 & 0.621 & $\mathrm{R}$ \\
\hline W3-702 & 0 & - & 216.224899 & 56.157280 & 0.106 & -21.47 & 10.33 & 0.300 & B \\
\hline W3-703 & 0 & - & 216.147308 & 56.249447 & 0.184 & -23.31 & 10.94 & 0.180 & B \\
\hline W3-704 & 0 & - & 216.716431 & 56.414154 & 0.115 & -21.77 & 10.66 & 0.483 & B \\
\hline W3-706 & 0 & - & 215.713120 & 56.523972 & 0.113 & -21.75 & 10.50 & 0.348 & B \\
\hline W3-708 & 2 & - & 215.834610 & 56.688942 & 0.150 & -22.48 & 11.12 & 0.642 & $\mathrm{R}$ \\
\hline W3-709 & 0 & - & 216.853760 & 56.725590 & 0.150 & -22.44 & 11.07 & 0.609 & $\mathrm{R}$ \\
\hline W3-710 & 0 & - & 215.305298 & 56.716385 & 0.088 & -21.40 & 10.48 & 0.461 & B \\
\hline W3-712 & 3 & M & 216.926880 & 55.908955 & 0.180 & -22.98 & 11.30 & 0.626 & $\mathrm{R}$ \\
\hline W3-713 & 0 & - & 217.849976 & 55.962254 & 0.160 & -22.68 & 10.91 & 0.379 & B \\
\hline W3-714 & 0 & - & 218.326538 & 56.001923 & 0.123 & -22.14 & 10.64 & 0.337 & B \\
\hline W3-716 & 0 & - & 217.678772 & 56.134270 & 0.072 & -20.73 & 10.07 & 0.328 & B \\
\hline W3-717 & 3 & M & 217.095627 & 56.231556 & 0.180 & -23.12 & 11.34 & 0.608 & $\mathrm{R}$ \\
\hline W3-718 & 0 & - & 217.401672 & 56.260201 & 0.123 & -22.24 & 10.80 & 0.444 & B \\
\hline W3-719 & 0 & - & 217.558807 & 56.393723 & 0.120 & -21.85 & 10.85 & 0.620 & $\mathrm{R}$ \\
\hline W3-720 & 0 & - & 218.292633 & 56.446789 & 0.080 & -21.60 & 10.42 & 0.334 & B \\
\hline W3-721 & 4 & $\mathrm{H}$ & 218.322647 & 56.457497 & 0.098 & -21.60 & 10.66 & 0.543 & $\mathrm{R}$ \\
\hline W3-722 & 2 & - & 218.273560 & 56.454361 & 0.067 & -20.57 & 10.30 & 0.590 & $\mathrm{R}$ \\
\hline W3-723 & 4 & $\mathrm{~F}, \mathrm{H}$ & 216.949448 & 56.550613 & 0.094 & -21.61 & 10.75 & 0.621 & $\mathrm{R}$ \\
\hline W3-724 & 2 & - & 218.330032 & 56.567432 & 0.070 & -21.03 & 10.50 & 0.607 & $\mathrm{R}$ \\
\hline W3-725 & 0 & - & 216.940140 & 56.570087 & 0.149 & -22.42 & 11.06 & 0.609 & $\mathrm{R}$ \\
\hline W3-727 & 0 & - & 216.926575 & 56.595989 & 0.147 & -22.51 & 10.82 & 0.366 & B \\
\hline
\end{tabular}


TABLE 4-Continued

\begin{tabular}{|c|c|c|c|c|c|c|c|c|c|}
\hline ID & Confidence $^{\mathrm{b}}$ & Features $^{c}$ & $\begin{array}{c}\text { RA } \\
{[\mathrm{J} 2000]}\end{array}$ & $\begin{array}{c}\text { Dec } \\
{[\mathrm{J} 2000]}\end{array}$ & $z_{\text {phot }}$ & $\begin{array}{c}\mathrm{M}_{r}^{\prime \mathrm{d}} \\
{[\mathrm{mag}]}\end{array}$ & $\begin{array}{c}\mathrm{M}_{\star}{ }^{\mathrm{e}} \\
{\left[\log \left(\mathrm{M}_{\odot}\right)\right]}\end{array}$ & $\begin{array}{c}\left(g^{\prime}-r^{\prime}\right)^{\mathrm{f}} \\
{[\mathrm{mag}]}\end{array}$ & Red/Blue ${ }^{\mathrm{g}}$ \\
\hline W3-728 & 0 & - & 217.336578 & 56.600349 & 0.150 & -22.85 & 11.23 & 0.612 & $\mathrm{R}$ \\
\hline W3-729 & 3 & $\mathrm{H}$ & 218.031906 & 56.618202 & 0.150 & -22.83 & 11.23 & 0.612 & $\mathrm{R}$ \\
\hline W3-730 & 0 & - & 217.152527 & 56.609116 & 0.153 & -22.54 & 11.11 & 0.610 & $\mathrm{R}$ \\
\hline W3-731 & 0 & - & 218.406113 & 56.629433 & 0.091 & -21.64 & 10.76 & 0.620 & $\mathrm{R}$ \\
\hline W3-732 & 0 & - & 217.798172 & 56.635506 & 0.098 & -21.41 & 10.26 & 0.255 & B \\
\hline W3-733 & 1 & - & 217.043900 & 56.663475 & 0.180 & -23.10 & 11.35 & 0.626 & $\mathrm{R}$ \\
\hline W3-734 & 0 & - & 217.337418 & 56.683056 & 0.150 & -22.63 & 11.14 & 0.605 & $\mathrm{R}$ \\
\hline W3-736 & 0 & - & 217.322952 & 56.729992 & 0.150 & -22.84 & 11.23 & 0.607 & $\mathrm{R}$ \\
\hline W3-737 & 0 & - & 218.031967 & 56.751259 & 0.119 & -21.80 & 10.69 & 0.497 & B \\
\hline W3-742 & 0 & - & 217.584915 & 56.799042 & 0.123 & -21.94 & 10.49 & 0.268 & B \\
\hline W3-743 & 2 & - & 216.934158 & 56.799492 & 0.129 & -22.27 & 11.03 & 0.635 & $\mathrm{R}$ \\
\hline W3-745 & 2 & - & 217.131683 & 56.834980 & 0.150 & -22.58 & 11.14 & 0.622 & $\mathrm{R}$ \\
\hline W3-746 & 4 & A & 219.654816 & 55.996777 & 0.110 & -21.90 & 10.60 & 0.383 & B \\
\hline W3-747 & 4 & $\mathrm{~F}$ & 219.660889 & 56.000236 & 0.142 & -22.43 & 10.84 & 0.410 & B \\
\hline W3-748 & 2 & - & 218.616119 & 55.997444 & 0.053 & -20.44 & 10.21 & 0.561 & $\mathrm{R}$ \\
\hline W3-749 & 4 & $\mathrm{~A}$ & 219.067215 & 56.028618 & 0.118 & -21.90 & 10.71 & 0.476 & B \\
\hline W3-750 & 1 & - & 219.713516 & 55.999035 & 0.157 & -22.91 & 11.06 & 0.431 & B \\
\hline W3-751 & 0 & - & 218.868317 & 56.005920 & 0.114 & -22.05 & 10.60 & 0.330 & B \\
\hline W3-752 & 0 & - & 219.332153 & 56.009197 & 0.103 & -21.48 & 10.31 & 0.276 & B \\
\hline W3-753 & 0 & - & 219.840378 & 56.031444 & 0.090 & -21.27 & 10.57 & 0.584 & $\mathrm{R}$ \\
\hline W3-755 & 0 & - & 219.492538 & 56.109177 & 0.180 & -22.87 & 11.22 & 0.594 & $\mathrm{R}$ \\
\hline W3-756 & 4 & $\mathrm{~A}, \mathrm{M}$ & 219.853424 & 56.118454 & 0.150 & -22.63 & 11.16 & 0.626 & $\mathrm{R}$ \\
\hline W3-757 & 4 & $\mathrm{~A}$ & 219.845901 & 56.122925 & 0.121 & -22.84 & 11.25 & 0.626 & $\mathrm{R}$ \\
\hline W3-758 & 0 & - & 219.977158 & 56.219234 & 0.115 & -21.83 & 10.54 & 0.353 & B \\
\hline W3-759 & 4 & $\mathrm{~S}, \mathrm{M}$ & 219.021851 & 56.254871 & 0.043 & -19.79 & 9.99 & 0.595 & $\mathrm{R}$ \\
\hline W3-760 & 0 & - & 219.337143 & 56.386539 & 0.150 & -22.77 & 11.18 & 0.588 & $\mathrm{R}$ \\
\hline W3-761 & 4 & $\mathrm{~L}$ & 218.698807 & 56.422020 & 0.091 & -21.50 & 10.68 & 0.602 & $\mathrm{R}$ \\
\hline W3-762 & 0 & - & 218.986176 & 56.435924 & 0.090 & -21.34 & 10.63 & 0.609 & $\mathrm{R}$ \\
\hline W3-763 & 0 & - & 219.847931 & 56.491878 & 0.105 & -21.97 & 10.71 & 0.458 & B \\
\hline W3-764 & 2 & - & 219.550812 & 56.497955 & 0.143 & -22.46 & 10.95 & 0.497 & B \\
\hline W3-765 & 2 & - & 219.590653 & 56.515137 & 0.120 & -22.16 & 10.84 & 0.504 & B \\
\hline W3-766 & 4 & $\mathrm{~S}$ & 220.072372 & 56.584400 & 0.121 & -22.14 & 10.97 & 0.626 & $\mathrm{R}$ \\
\hline W3-767 & 2 & - & 219.085083 & 56.594688 & 0.112 & -21.64 & 10.62 & 0.493 & B \\
\hline W3-768 & 1 & - & 218.756180 & 56.628857 & 0.101 & -22.18 & 10.81 & 0.470 & B \\
\hline W3-769 & 0 & - & 218.784302 & 56.658211 & 0.099 & -21.42 & 10.51 & 0.470 & B \\
\hline W3-770 & 0 & - & 219.757355 & 56.748199 & 0.180 & -23.42 & 11.48 & 0.628 & $\mathrm{R}$ \\
\hline W3-773 & 0 & - & 219.904556 & 56.840286 & 0.090 & -21.37 & 10.65 & 0.623 & $\mathrm{R}$ \\
\hline W3-774 & 0 & - & 209.539948 & 56.869362 & 0.062 & -20.63 & 10.33 & 0.600 & $\mathrm{R}$ \\
\hline W3-775 & 0 & - & 208.991562 & 56.884029 & 0.118 & -21.73 & 10.37 & 0.235 & B \\
\hline W3-776 & 0 & - & 208.783035 & 56.894363 & 0.060 & -20.56 & 10.36 & 0.650 & $\mathrm{R}$ \\
\hline W3-777 & 0 & - & 210.242432 & 56.976021 & 0.092 & -21.87 & 10.84 & 0.607 & $\mathrm{R}$ \\
\hline W3-780 & 1 & - & 209.932266 & 57.126465 & 0.083 & -21.39 & 10.56 & 0.536 & $\mathrm{R}$ \\
\hline W3-781 & 0 & - & 209.666199 & 57.156979 & 0.148 & -22.38 & 11.02 & 0.583 & $\mathrm{R}$ \\
\hline W3-783 & 0 & - & 209.068146 & 57.380859 & 0.152 & -22.53 & 11.14 & 0.646 & $\mathrm{R}$ \\
\hline
\end{tabular}


TABLE 4-Continued

\begin{tabular}{|c|c|c|c|c|c|c|c|c|c|}
\hline ID & Confidence $^{\mathrm{b}}$ & Features $^{\mathrm{c}}$ & $\begin{array}{c}\text { RA } \\
{[\mathrm{J} 2000]}\end{array}$ & $\begin{array}{c}\text { Dec } \\
{[\mathrm{J} 2000]}\end{array}$ & $z_{\text {phot }}$ & $\begin{array}{c}\mathrm{M}_{r}^{\prime \mathrm{d}} \\
{[\mathrm{mag}]}\end{array}$ & $\begin{array}{c}\mathrm{M}_{\star}{ }^{\mathrm{e}} \\
{\left[\log \left(\mathrm{M}_{\odot}\right)\right]}\end{array}$ & $\begin{array}{c}\left(g^{\prime}-r^{\prime}\right)^{\mathrm{f}} \\
{[\mathrm{mag}]}\end{array}$ & Red/Blue ${ }^{\mathrm{g}}$ \\
\hline W3-784 & 0 & - & 210.201584 & 57.511112 & 0.150 & -22.55 & 11.12 & 0.617 & $\mathrm{R}$ \\
\hline W3-785 & 0 & - & 209.112412 & 57.512756 & 0.084 & -21.42 & 10.66 & 0.610 & $\mathrm{R}$ \\
\hline W3-786 & 0 & - & 208.690842 & 57.556175 & 0.093 & -21.59 & 10.46 & 0.371 & B \\
\hline W3-787 & 0 & - & 210.047195 & 57.640346 & 0.124 & -22.43 & 11.09 & 0.627 & $\mathrm{R}$ \\
\hline W3-788 & 1 & - & 210.170654 & 57.664898 & 0.140 & -22.80 & 11.02 & 0.438 & B \\
\hline W3-789 & 0 & - & 209.080978 & 57.709969 & 0.180 & -22.90 & 11.25 & 0.606 & $\mathrm{R}$ \\
\hline W3-791 & 0 & - & 210.780258 & 57.065758 & 0.130 & -21.99 & 10.71 & 0.444 & B \\
\hline W3-792 & 0 & - & 210.629623 & 57.093540 & 0.078 & -21.44 & 10.64 & 0.581 & $\mathrm{R}$ \\
\hline W3-793 & 1 & - & 211.104095 & 57.118259 & 0.153 & -23.04 & 11.32 & 0.618 & $\mathrm{R}$ \\
\hline W3-794 & 0 & - & 211.904373 & 57.227856 & 0.125 & -22.17 & 10.70 & 0.377 & B \\
\hline W3-795 & 0 & - & 211.849747 & 57.411072 & 0.091 & -21.33 & 10.20 & 0.225 & B \\
\hline W3-797 & 0 & - & 211.478439 & 57.549408 & 0.086 & -21.28 & 10.20 & 0.246 & B \\
\hline W3-798 & 0 & - & 211.546204 & 57.589668 & 0.088 & -21.12 & 10.51 & 0.581 & $\mathrm{R}$ \\
\hline W3-800 & 0 & - & 211.546249 & 57.619793 & 0.068 & -20.55 & 10.00 & 0.333 & B \\
\hline W3-801 & 1 & - & 210.602402 & 57.656265 & 0.128 & -22.00 & 10.66 & 0.397 & B \\
\hline W3-802 & 0 & - & 210.611969 & 57.661480 & 0.180 & -23.29 & 11.42 & 0.616 & $\mathrm{R}$ \\
\hline W3-803 & 0 & - & 210.684494 & 57.714790 & 0.140 & -22.38 & 10.96 & 0.529 & B \\
\hline W3-805 & 4 & $\mathrm{~S}$ & 212.316834 & 56.859882 & 0.150 & -22.71 & 11.20 & 0.625 & $\mathrm{R}$ \\
\hline W3-807 & 0 & - & 212.169556 & 56.905048 & 0.180 & -23.25 & 11.39 & 0.603 & $\mathrm{R}$ \\
\hline W3-808 & 0 & - & 212.281433 & 56.940502 & 0.132 & -22.26 & 10.98 & 0.595 & $\mathrm{R}$ \\
\hline W3-809 & 1 & - & 212.119080 & 56.946087 & 0.150 & -22.55 & 11.10 & 0.601 & $\mathrm{R}$ \\
\hline W3-810 & 0 & - & 212.256744 & 56.980030 & 0.155 & -22.98 & 11.07 & 0.421 & B \\
\hline W3-811 & 0 & - & 212.578171 & 57.017670 & 0.121 & -22.22 & 11.08 & 0.700 & $\mathrm{R}$ \\
\hline W3-812 & 0 & - & 212.510757 & 57.019852 & 0.180 & -22.99 & 11.27 & 0.594 & $\mathrm{R}$ \\
\hline W3-813 & 0 & - & 212.269653 & 57.037430 & 0.112 & -21.86 & 10.50 & 0.306 & B \\
\hline W3-814 & 0 & - & 212.885895 & 57.068798 & 0.128 & -22.03 & 10.91 & 0.610 & $\mathrm{R}$ \\
\hline W3-815 & 2 & - & 212.095871 & 57.147739 & 0.102 & -21.77 & 10.59 & 0.421 & B \\
\hline W3-816 & 4 & M & 213.139252 & 57.179367 & 0.150 & -22.50 & 11.07 & 0.591 & $\mathrm{R}$ \\
\hline W3-817 & 0 & - & 212.920578 & 57.198368 & 0.150 & -23.00 & 11.24 & 0.561 & $\mathrm{R}$ \\
\hline W3-818 & 0 & - & 212.458023 & 57.225040 & 0.069 & -20.80 & 10.06 & 0.298 & B \\
\hline W3-819 & 0 & - & 213.491364 & 57.238537 & 0.121 & -22.01 & 10.74 & 0.464 & B \\
\hline W3-820 & 0 & - & 213.526031 & 57.333084 & 0.134 & -22.16 & 10.92 & 0.574 & $\mathrm{R}$ \\
\hline W3-821 & 0 & - & 213.014008 & 57.363293 & 0.124 & -22.40 & 10.88 & 0.452 & B \\
\hline W3-822 & 0 & - & 212.613205 & 57.366070 & 0.120 & -22.19 & 10.92 & 0.567 & $\mathrm{R}$ \\
\hline W3-823 & 0 & - & 213.576584 & 57.377564 & 0.099 & -21.48 & 10.46 & 0.412 & B \\
\hline W3-824 & 0 & - & 212.130005 & 57.406738 & 0.102 & -21.94 & 10.84 & 0.580 & $\mathrm{R}$ \\
\hline W3-825 & 1 & - & 212.758179 & 57.428154 & 0.150 & -22.50 & 11.09 & 0.610 & $\mathrm{R}$ \\
\hline W3-826 & 0 & - & 212.314529 & 57.446087 & 0.095 & -21.46 & 10.61 & 0.548 & $\mathrm{R}$ \\
\hline W3-827 & 2 & - & 212.966934 & 57.460319 & 0.179 & -23.38 & 11.41 & 0.583 & $\mathrm{R}$ \\
\hline W3-828 & 4 & $\mathrm{~S}$ & 213.367996 & 57.476372 & 0.085 & -21.01 & 10.37 & 0.496 & B \\
\hline W3-829 & 0 & - & 212.964462 & 57.488709 & 0.150 & -22.80 & 11.20 & 0.599 & $\mathrm{R}$ \\
\hline W3-830 & 0 & - & 213.345520 & 57.518585 & 0.174 & -22.94 & 11.26 & 0.602 & $\mathrm{R}$ \\
\hline W3-831 & 0 & - & 212.824158 & 57.520996 & 0.130 & -22.04 & 10.78 & 0.490 & B \\
\hline W3-832 & 4 & M & 213.586731 & 57.546841 & 0.150 & -22.79 & 11.22 & 0.619 & $\mathrm{R}$ \\
\hline
\end{tabular}


TABLE 4-Continued

\begin{tabular}{|c|c|c|c|c|c|c|c|c|c|}
\hline ID & Confidence $^{\mathrm{b}}$ & Features $^{\mathrm{c}}$ & $\begin{array}{c}\text { RA } \\
{[\mathrm{J} 2000]}\end{array}$ & $\begin{array}{c}\text { Dec } \\
{[\mathrm{J} 2000]}\end{array}$ & $z_{\text {phot }}$ & $\begin{array}{c}\mathrm{M}_{r}^{\prime \mathrm{d}} \\
{[\mathrm{mag}]}\end{array}$ & $\begin{array}{c}\mathrm{M}_{\star}{ }^{\mathrm{e}} \\
{\left[\log \left(\mathrm{M}_{\odot}\right)\right]}\end{array}$ & $\begin{array}{c}\left(g^{\prime}-r^{\prime}\right)^{\mathrm{f}} \\
{[\mathrm{mag}]}\end{array}$ & Red/Blue ${ }^{\mathrm{g}}$ \\
\hline W3-833 & 0 & - & 213.546829 & 57.544617 & 0.120 & -21.98 & 10.89 & 0.610 & $\mathrm{R}$ \\
\hline W3-834 & 0 & - & 213.604874 & 57.630859 & 0.130 & -22.69 & 10.91 & 0.381 & B \\
\hline W3-835 & 2 & - & 212.070816 & 57.652313 & 0.151 & -22.50 & 11.11 & 0.624 & $\mathrm{R}$ \\
\hline W3-837 & 0 & - & 212.748459 & 57.691952 & 0.096 & -21.59 & 10.70 & 0.581 & $\mathrm{R}$ \\
\hline W3-838 & 0 & - & 212.352661 & 57.716354 & 0.107 & -21.81 & 10.64 & 0.450 & B \\
\hline W3-839 & 0 & - & 212.743149 & 57.759705 & 0.180 & -23.07 & 11.33 & 0.617 & $\mathrm{R}$ \\
\hline W3-840 & 1 & - & 212.720154 & 57.768215 & 0.180 & -23.47 & 11.52 & 0.645 & $\mathrm{R}$ \\
\hline W3-841 & 1 & - & 212.464600 & 57.796677 & 0.150 & -22.96 & 11.30 & 0.629 & $\mathrm{R}$ \\
\hline W3-842 & 3 & $\mathrm{~A}$ & 215.197632 & 56.852032 & 0.150 & -23.01 & 11.31 & 0.616 & $\mathrm{R}$ \\
\hline W3-843 & 0 & - & 215.117325 & 56.857895 & 0.067 & -20.50 & 10.21 & 0.533 & $\mathrm{R}$ \\
\hline W3-844 & 0 & - & 215.214371 & 56.872746 & 0.074 & -20.78 & 10.25 & 0.468 & B \\
\hline W3-846 & 3 & M & 213.734436 & 56.902283 & 0.180 & -22.86 & 11.22 & 0.594 & $\mathrm{R}$ \\
\hline W3-849 & 0 & - & 215.118744 & 57.017494 & 0.090 & -21.16 & 10.45 & 0.518 & $\mathrm{R}$ \\
\hline W3-850 & 0 & - & 214.204330 & 57.047108 & 0.150 & -22.51 & 11.09 & 0.597 & $\mathrm{R}$ \\
\hline W3-851 & 0 & - & 213.661041 & 57.198566 & 0.150 & -22.55 & 11.12 & 0.617 & $\mathrm{R}$ \\
\hline W3-852 & 0 & - & 214.466675 & 57.235199 & 0.100 & -21.89 & 10.79 & 0.553 & $\mathrm{R}$ \\
\hline W3-853 & 0 & - & 214.270615 & 57.254372 & 0.067 & -20.98 & 10.42 & 0.553 & $\mathrm{R}$ \\
\hline W3-855 & 0 & - & 214.327591 & 57.362904 & 0.056 & -20.41 & 10.05 & 0.422 & B \\
\hline W3-856 & 0 & - & 214.210739 & 57.393139 & 0.129 & -22.49 & 10.89 & 0.428 & B \\
\hline W3-857 & 1 & - & 214.539276 & 57.518600 & 0.098 & -21.66 & 10.69 & 0.546 & $\mathrm{R}$ \\
\hline W3-858 & 0 & - & 214.464539 & 57.531799 & 0.150 & -22.90 & 11.21 & 0.574 & $\mathrm{R}$ \\
\hline W3-860 & 0 & - & 213.864471 & 57.609169 & 0.118 & -22.46 & 10.84 & 0.399 & B \\
\hline W3-861 & 0 & - & 213.954086 & 57.662121 & 0.126 & -22.66 & 10.93 & 0.404 & B \\
\hline W3-866 & 0 & - & 215.365021 & 57.005806 & 0.125 & -22.47 & 10.98 & 0.515 & B \\
\hline W3-867 & 0 & - & 216.624939 & 57.024589 & 0.087 & -21.02 & 10.21 & 0.354 & B \\
\hline W3-868 & 4 & $\mathrm{~L}$ & 216.099915 & 57.029461 & 0.102 & -21.46 & 10.24 & 0.219 & B \\
\hline W3-869 & 0 & - & 216.254379 & 57.065247 & 0.121 & -21.91 & 10.85 & 0.601 & $\mathrm{R}$ \\
\hline W3-872 & 4 & $\mathrm{~A}$ & 216.799850 & 57.140450 & 0.099 & -21.70 & 10.35 & 0.233 & B \\
\hline W3-873 & 4 & $\mathrm{~A}$ & 216.820038 & 57.143543 & 0.095 & -21.31 & 10.03 & 0.085 & B \\
\hline W3-874 & 2 & - & 215.753754 & 57.178761 & 0.097 & -21.40 & 10.44 & 0.424 & B \\
\hline W3-875 & 0 & - & 215.837585 & 57.231533 & 0.130 & -22.06 & 10.66 & 0.379 & B \\
\hline W3-876 & 0 & - & 216.839279 & 57.206802 & 0.127 & -22.03 & 10.63 & 0.364 & B \\
\hline W3-877 & 0 & - & 216.341614 & 57.265285 & 0.127 & -22.45 & 10.86 & 0.421 & B \\
\hline W3-878 & 0 & - & 216.529862 & 57.289738 & 0.090 & -21.72 & 10.80 & 0.625 & $\mathrm{R}$ \\
\hline W3-879 & 0 & - & 215.591431 & 57.299217 & 0.121 & -22.16 & 10.72 & 0.399 & B \\
\hline W3-880 & 0 & - & 215.376602 & 57.295055 & 0.073 & -21.00 & 10.46 & 0.577 & $\mathrm{R}$ \\
\hline W3-881 & 4 & $\mathrm{~F}$ & 216.952270 & 57.630280 & 0.121 & -21.86 & 10.85 & 0.621 & $\mathrm{R}$ \\
\hline W3-886 & 0 & - & 217.328995 & 56.873768 & 0.117 & -21.93 & 10.82 & 0.570 & $\mathrm{R}$ \\
\hline W3-887 & 0 & - & 217.512787 & 56.904774 & 0.180 & -23.02 & 11.29 & 0.597 & $\mathrm{R}$ \\
\hline W3-889 & 2 & - & 217.637878 & 56.925568 & 0.180 & -23.18 & 11.36 & 0.602 & $\mathrm{R}$ \\
\hline W3-890 & 0 & - & 218.177978 & 56.964821 & 0.092 & -21.50 & 10.68 & 0.593 & $\mathrm{R}$ \\
\hline W3-891 & 0 & - & 217.117416 & 56.988270 & 0.140 & -22.28 & 10.99 & 0.598 & $\mathrm{R}$ \\
\hline W3-892 & 1 & - & 216.981613 & 57.009777 & 0.161 & -23.01 & 11.29 & 0.602 & $\mathrm{R}$ \\
\hline W3-893 & 3 & $\mathrm{~A}$ & 218.316864 & 57.014088 & 0.180 & -23.35 & 11.42 & 0.595 & $\mathrm{R}$ \\
\hline
\end{tabular}


TABLE 4-Continued

\begin{tabular}{|c|c|c|c|c|c|c|c|c|c|}
\hline ID & Confidence $^{\mathrm{b}}$ & Features $^{c}$ & $\begin{array}{c}\text { RA } \\
{[\mathrm{J} 2000]}\end{array}$ & $\begin{array}{c}\text { Dec } \\
{[\mathrm{J} 2000]}\end{array}$ & $z_{\text {phot }}$ & $\begin{array}{c}\mathrm{M}_{r}^{\prime \mathrm{d}} \\
{[\mathrm{mag}]}\end{array}$ & $\begin{array}{c}\mathrm{M}_{\star}{ }^{\mathrm{e}} \\
{\left[\log \left(\mathrm{M}_{\odot}\right)\right]}\end{array}$ & $\begin{array}{c}\left(g^{\prime}-r^{\prime}\right)^{\mathrm{f}} \\
{[\mathrm{mag}]}\end{array}$ & Red/Blue ${ }^{\mathrm{g}}$ \\
\hline W3-894 & 0 & - & 217.003388 & 57.012856 & 0.157 & -22.97 & 11.30 & 0.631 & $\mathrm{R}$ \\
\hline W3-895 & 0 & - & 218.059280 & 57.140106 & 0.183 & -22.78 & 10.59 & 0.057 & B \\
\hline W3-896 & 0 & - & 218.260208 & 57.216923 & 0.105 & -21.65 & 10.59 & 0.467 & B \\
\hline W3-897 & 3 & $\mathrm{~A}$ & 217.076630 & 57.228882 & 0.129 & -22.11 & 10.50 & 0.218 & B \\
\hline W3-898 & 0 & - & 217.817062 & 57.279854 & 0.151 & -22.35 & 10.52 & 0.152 & B \\
\hline W3-899 & 0 & - & 217.817184 & 57.344814 & 0.150 & -22.48 & 11.08 & 0.600 & $\mathrm{R}$ \\
\hline W3-900 & 0 & - & 217.765915 & 57.341679 & 0.097 & -21.64 & 10.65 & 0.521 & $\mathrm{R}$ \\
\hline W3-901 & 0 & - & 217.781281 & 57.350887 & 0.150 & -22.72 & 11.17 & 0.600 & $\mathrm{R}$ \\
\hline W3-902 & 1 & - & 217.740753 & 57.373817 & 0.114 & -21.90 & 10.81 & 0.568 & $\mathrm{R}$ \\
\hline W3-903 & 0 & - & 218.434753 & 57.407360 & 0.071 & -20.62 & 10.08 & 0.375 & B \\
\hline W3-904 & 0 & - & 218.383194 & 57.408386 & 0.183 & -22.95 & 11.02 & 0.386 & B \\
\hline W3-905 & 4 & $\mathrm{~A}, \mathrm{~L}$ & 217.796539 & 57.438427 & 0.102 & -22.07 & 10.83 & 0.530 & $\mathrm{R}$ \\
\hline W3-906 & 0 & - & 218.517960 & 57.420505 & 0.150 & -22.60 & 11.15 & 0.624 & $\mathrm{R}$ \\
\hline W3-907 & 4 & - & 217.814926 & 57.437256 & 0.126 & -22.05 & 10.88 & 0.583 & $\mathrm{R}$ \\
\hline W3-908 & 0 & - & 217.854416 & 57.469807 & 0.077 & -21.00 & 10.33 & 0.461 & B \\
\hline W3-909 & 3 & M & 218.543610 & 57.459923 & 0.175 & -23.17 & 11.35 & 0.600 & $\mathrm{R}$ \\
\hline W3-910 & 4 & $\mathrm{~L}$ & 218.565414 & 57.471237 & 0.159 & -22.65 & 11.15 & 0.603 & $\mathrm{R}$ \\
\hline W3-911 & 0 & - & 218.466888 & 57.494324 & 0.121 & -22.28 & 10.95 & 0.560 & $\mathrm{R}$ \\
\hline W3-912 & 0 & - & 218.329498 & 57.510162 & 0.152 & -22.84 & 11.22 & 0.603 & $\mathrm{R}$ \\
\hline W3-914 & 0 & - & 217.852417 & 57.634521 & 0.093 & -21.59 & 10.65 & 0.540 & $\mathrm{R}$ \\
\hline W3-916 & 0 & - & 217.859497 & 57.715527 & 0.115 & -22.14 & 10.89 & 0.552 & $\mathrm{R}$ \\
\hline W3-919 & 1 & - & 219.293518 & 56.898220 & 0.102 & -21.76 & 10.56 & 0.396 & B \\
\hline W3-922 & 0 & - & 219.630402 & 57.205872 & 0.094 & -21.54 & 10.74 & 0.633 & $\mathrm{R}$ \\
\hline W3-923 & 1 & - & 218.938782 & 57.271553 & 0.158 & -23.22 & 11.34 & 0.571 & $\mathrm{R}$ \\
\hline W3-926 & 0 & - & 219.819427 & 57.313759 & 0.076 & -21.17 & 10.27 & 0.349 & B \\
\hline W3-927 & 0 & - & 219.972611 & 57.319660 & 0.123 & -22.16 & 10.79 & 0.462 & B \\
\hline W3-928 & 0 & - & 219.187576 & 57.342899 & 0.163 & -22.62 & 11.18 & 0.645 & $\mathrm{R}$ \\
\hline W3-930 & 0 & - & 219.250748 & 57.431534 & 0.121 & -21.87 & 10.69 & 0.477 & B \\
\hline W3-931 & 0 & - & 220.118393 & 57.482555 & 0.078 & -20.79 & 10.13 & 0.359 & B \\
\hline W3-932 & 0 & - & 218.708664 & 57.585598 & 0.093 & -21.59 & 10.50 & 0.410 & B \\
\hline W3-933 & 0 & - & 219.338120 & 57.601242 & 0.106 & -21.83 & 10.46 & 0.282 & B \\
\hline W3-934 & 1 & - & 219.975433 & 57.683632 & 0.088 & -21.20 & 10.21 & 0.284 & B \\
\hline W4-1 & 3 & $\mathrm{~A}$ & 332.187439 & -0.985447 & 0.196 & -23.71 & 11.40 & 0.456 & B \\
\hline W4-2 & 0 & - & 332.128876 & -0.945157 & 0.123 & -21.84 & 10.55 & 0.363 & B \\
\hline W4-3 & 0 & - & 331.939850 & -0.868295 & 0.171 & -22.84 & 11.04 & 0.437 & B \\
\hline W4-4 & 3 & $\mathrm{M}$ & 332.631531 & -0.841735 & 0.096 & -21.37 & 10.31 & 0.317 & B \\
\hline W4-5 & 4 & $\mathrm{~S}, \mathrm{~L}$ & 332.562439 & -0.842626 & 0.180 & -23.09 & 11.34 & 0.615 & $\mathrm{R}$ \\
\hline W4-6 & 4 & $\mathrm{~L}$ & 332.772156 & -0.801952 & 0.093 & -21.45 & 10.48 & 0.442 & B \\
\hline W4-7 & 0 & - & 331.924866 & -0.765838 & 0.148 & -23.41 & 11.26 & 0.431 & B \\
\hline W4-8 & 2 & - & 332.055695 & -0.722331 & 0.128 & -22.28 & 11.05 & 0.648 & $\mathrm{R}$ \\
\hline W4-11 & 0 & - & 332.290710 & -0.651755 & 0.060 & -20.49 & 10.31 & 0.631 & $\mathrm{R}$ \\
\hline W4-12 & 0 & - & 332.120697 & -0.573062 & 0.136 & -22.29 & 11.04 & 0.638 & $\mathrm{R}$ \\
\hline W4-13 & 0 & - & 332.066833 & -0.560870 & 0.193 & -23.28 & 11.22 & 0.444 & B \\
\hline W4-14 & 0 & - & 332.426392 & -0.561766 & 0.128 & -22.37 & 10.79 & 0.380 & B \\
\hline
\end{tabular}


TABLE 4-Continued

\begin{tabular}{|c|c|c|c|c|c|c|c|c|c|}
\hline ID & Confidence $^{\mathrm{b}}$ & Features $^{\mathrm{c}}$ & $\begin{array}{c}\text { RA } \\
{[\mathrm{J} 2000]}\end{array}$ & $\begin{array}{c}\text { Dec } \\
{[\mathrm{J} 2000]}\end{array}$ & $z_{\text {phot }}$ & $\begin{array}{c}\mathrm{M}_{r}^{\prime \mathrm{d}} \\
{[\mathrm{mag}]}\end{array}$ & $\begin{array}{c}\mathrm{M}_{\star}^{\mathrm{e}} \\
{\left[\log \left(\mathrm{M}_{\odot}\right)\right]}\end{array}$ & $\begin{array}{c}\left(g^{\prime}-r^{\prime}\right)^{\mathrm{f}} \\
{[\mathrm{mag}]}\end{array}$ & Red/Blue ${ }^{\mathrm{g}}$ \\
\hline W4-15 & 0 & - & 332.380981 & -0.507227 & 0.180 & -23.21 & 11.39 & 0.626 & $\mathrm{R}$ \\
\hline W4-16 & 1 & - & 332.423340 & -0.343151 & 0.149 & -22.62 & 11.14 & 0.608 & $\mathrm{R}$ \\
\hline W4-20 & 0 & - & 332.376892 & -0.232011 & 0.165 & -22.85 & 11.01 & 0.411 & $\mathrm{~B}$ \\
\hline W4-21 & 4 & $\mathrm{~L}$ & 332.015442 & -0.249663 & 0.162 & -22.64 & 11.04 & 0.509 & B \\
\hline W4-22 & 0 & - & 332.469452 & -0.245198 & 0.163 & -22.66 & 11.08 & 0.535 & $\mathrm{R}$ \\
\hline W4-23 & 0 & - & 332.378540 & -0.153945 & 0.062 & -20.29 & 10.22 & 0.617 & $\mathrm{R}$ \\
\hline W4-25 & 4 & $\mathrm{~A}, \mathrm{M}$ & 332.277710 & -0.095191 & 0.198 & -23.02 & 11.12 & 0.446 & $\mathrm{~B}$ \\
\hline W4-27 & 0 & - & 333.094574 & -0.962172 & 0.180 & -23.20 & 11.38 & 0.617 & $\mathrm{R}$ \\
\hline W4-29 & 0 & - & 332.996338 & -0.866336 & 0.120 & -21.96 & 10.84 & 0.573 & $\mathrm{R}$ \\
\hline W4-30 & 0 & - & 333.135864 & -0.868342 & 0.094 & -21.53 & 10.29 & 0.233 & $\mathrm{~B}$ \\
\hline W4-32 & 4 & - & 332.969147 & -0.827915 & 0.139 & -22.54 & 11.04 & 0.551 & $\mathrm{R}$ \\
\hline W4-33 & 0 & - & 333.410278 & -0.659344 & 0.135 & -22.72 & 11.19 & 0.616 & $\mathrm{R}$ \\
\hline W4-34 & 0 & - & 333.599792 & -0.616455 & 0.149 & -23.02 & 11.26 & 0.573 & $\mathrm{R}$ \\
\hline W4-38 & 1 & - & 332.881928 & -0.481501 & 0.145 & -22.51 & 11.07 & 0.583 & $\mathrm{R}$ \\
\hline W4-40 & 0 & - & 332.980469 & -0.293282 & 0.150 & -22.77 & 11.25 & 0.652 & $\mathrm{R}$ \\
\hline W4-42 & 0 & - & 333.719879 & -0.189874 & 0.084 & -21.32 & 10.49 & 0.493 & $\mathrm{~B}$ \\
\hline W4-43 & 1 & - & 333.296814 & -0.188359 & 0.150 & -23.10 & 11.36 & 0.630 & $\mathrm{R}$ \\
\hline W4-46 & 0 & - & 333.924530 & -0.977121 & 0.112 & -21.83 & 10.60 & 0.412 & $\mathrm{~B}$ \\
\hline W4-47 & 4 & $\mathrm{~A}$ & 334.168213 & -0.967574 & 0.109 & -21.93 & 10.63 & 0.396 & B \\
\hline W4-48 & 1 & - & 334.683624 & -0.949678 & 0.050 & -20.39 & 10.21 & 0.577 & $\mathrm{R}$ \\
\hline W4-49 & 0 & - & 334.678040 & -0.909993 & 0.096 & -21.43 & 10.64 & 0.592 & $\mathrm{R}$ \\
\hline W4-50 & 2 & - & 334.294983 & -0.835698 & 0.120 & -22.39 & 11.02 & 0.584 & $\mathrm{R}$ \\
\hline W4-51 & 4 & M & 334.142487 & -0.743079 & 0.095 & -21.36 & 10.62 & 0.589 & $\mathrm{R}$ \\
\hline W4-52 & 0 & - & 334.700775 & -0.625146 & 0.120 & -22.39 & 11.02 & 0.584 & $\mathrm{R}$ \\
\hline W4-54 & 4 & $\mathrm{~A}, \mathrm{~L}, \mathrm{H}$ & 334.127228 & -0.574382 & 0.123 & -22.13 & 10.61 & 0.306 & $\mathrm{~B}$ \\
\hline W4-55 & 0 & - & 334.634705 & -0.500728 & 0.152 & -23.19 & 11.37 & 0.612 & $\mathrm{R}$ \\
\hline W4-56 & 4 & $\mathrm{H}$ & 334.054413 & -0.486237 & 0.150 & -23.15 & 11.33 & 0.592 & $\mathrm{R}$ \\
\hline W4-57 & 2 & - & 334.295715 & -0.460554 & 0.097 & -21.85 & 10.50 & 0.308 & B \\
\hline W4-58 & 4 & $\mathrm{~S}, \mathrm{~A}$ & 334.636993 & -0.470263 & 0.117 & -22.08 & 10.84 & 0.529 & $\mathrm{R}$ \\
\hline W4-59 & 0 & - & 334.173462 & -0.451006 & 0.157 & -23.07 & 11.10 & 0.411 & $\mathrm{~B}$ \\
\hline W4-62 & 0 & - & 334.035675 & -0.342842 & 0.180 & -23.49 & 11.49 & 0.615 & $\mathrm{R}$ \\
\hline W4-63 & 0 & - & 334.428925 & -0.290699 & 0.141 & -22.13 & 10.58 & 0.281 & $\mathrm{~B}$ \\
\hline W4-65 & 0 & - & 334.342377 & -0.268455 & 0.116 & -21.79 & 10.55 & 0.383 & $\mathrm{~B}$ \\
\hline W4-66 & 0 & - & 334.191437 & -0.260520 & 0.078 & -21.36 & 10.58 & 0.560 & $\mathrm{R}$ \\
\hline W4-67 & 0 & - & 334.296143 & -0.258003 & 0.056 & -20.17 & 10.11 & 0.569 & $\mathrm{R}$ \\
\hline W4-68 & 0 & - & 334.196075 & -0.182738 & 0.150 & -23.02 & 11.30 & 0.612 & $\mathrm{R}$ \\
\hline W4-70 & 0 & - & 334.252869 & -0.080870 & 0.120 & -22.04 & 10.91 & 0.609 & $\mathrm{R}$ \\
\hline W4-73 & 0 & - & 334.899872 & -1.001287 & 0.073 & -21.27 & 10.43 & 0.461 & $\mathrm{~B}$ \\
\hline W4-74 & 0 & - & 335.067474 & -1.004113 & 0.131 & -22.73 & 10.95 & 0.401 & $\mathrm{~B}$ \\
\hline W4-75 & 0 & - & 334.830566 & -0.977966 & 0.122 & -22.22 & 11.02 & 0.646 & $\mathrm{R}$ \\
\hline W4-76 & 0 & - & 334.903686 & -0.985897 & 0.075 & -20.80 & 10.23 & 0.449 & $\mathrm{~B}$ \\
\hline W4-77 & 1 & - & 335.435913 & -0.926112 & 0.083 & -21.10 & 10.46 & 0.546 & $\mathrm{R}$ \\
\hline W4-78 & 0 & - & 335.653870 & -0.894193 & 0.120 & -22.00 & 10.56 & 0.318 & $\mathrm{~B}$ \\
\hline W4-79 & 0 & - & 334.909454 & -0.872048 & 0.085 & -21.62 & 10.50 & 0.394 & $\mathrm{~B}$ \\
\hline
\end{tabular}


TABlE 4-Continued

\begin{tabular}{|c|c|c|c|c|c|c|c|c|c|}
\hline ID & Confidence $^{\mathrm{b}}$ & Features $^{\mathrm{c}}$ & $\begin{array}{c}\text { RA } \\
{[\mathrm{J} 2000]}\end{array}$ & $\begin{array}{c}\text { Dec } \\
{[\mathrm{J} 2000]}\end{array}$ & $z_{p h o t}$ & $\begin{array}{c}\mathrm{M}_{r}^{\prime \mathrm{d}} \\
{[\mathrm{mag}]}\end{array}$ & $\begin{array}{c}\mathrm{M}_{\star}^{\mathrm{e}} \\
{\left[\log \left(\mathrm{M}_{\odot}\right)\right]}\end{array}$ & $\begin{array}{c}\left(g^{\prime}-r^{\prime}\right)^{\mathrm{f}} \\
{[\mathrm{mag}]}\end{array}$ & Red/Blue ${ }^{\mathrm{g}}$ \\
\hline W4-80 & 0 & - & 334.790100 & -0.851103 & 0.120 & -22.20 & 11.03 & 0.657 & $\mathrm{R}$ \\
\hline W4-81 & 1 & - & 335.395538 & -0.813621 & 0.180 & -23.08 & 11.35 & 0.634 & $\mathrm{R}$ \\
\hline W4-82 & 4 & $\mathrm{~A}$ & 334.950348 & -0.802236 & 0.136 & -22.20 & 10.88 & 0.524 & $\mathrm{~B}$ \\
\hline W4-83 & 2 & - & 334.881897 & -0.700974 & 0.161 & -23.08 & 11.13 & 0.435 & B \\
\hline W4-84 & 0 & - & 335.401184 & -0.660927 & 0.093 & -21.36 & 10.43 & 0.421 & $\mathrm{~B}$ \\
\hline W4-89 & 0 & - & 335.057007 & -0.456164 & 0.067 & -20.45 & 10.22 & 0.561 & $\mathrm{R}$ \\
\hline W4-90 & 0 & - & 334.976318 & -0.438360 & 0.182 & -23.42 & 11.43 & 0.584 & $\mathrm{R}$ \\
\hline W4-92 & 4 & $\mathrm{~A}$ & 335.277344 & -0.393877 & 0.103 & -22.04 & 10.82 & 0.531 & $\mathrm{R}$ \\
\hline W4-98 & 0 & - & 332.133514 & 0.094452 & 0.152 & -22.94 & 11.29 & 0.624 & $\mathrm{R}$ \\
\hline W4-103 & 4 & $\mathrm{~F}, \mathrm{M}$ & 332.065460 & 0.630648 & 0.144 & -23.17 & 11.36 & 0.604 & $\mathrm{R}$ \\
\hline W4-104 & 0 & - & 332.662415 & 0.649555 & 0.149 & -22.61 & 11.06 & 0.542 & $\mathrm{R}$ \\
\hline W4-108 & 0 & - & 333.260864 & -0.051349 & 0.121 & -21.96 & 10.90 & 0.628 & $\mathrm{R}$ \\
\hline W4-109 & 0 & - & 333.217316 & -0.016020 & 0.150 & -23.02 & 11.34 & 0.642 & $\mathrm{R}$ \\
\hline W4-110 & 0 & - & 333.428742 & 0.038930 & 0.150 & -22.42 & 11.00 & 0.558 & $\mathrm{R}$ \\
\hline W4-111 & 3 & $\mathrm{~A}, \mathrm{~F}$ & 332.951294 & 0.080042 & 0.115 & -21.70 & 10.54 & 0.401 & $\mathrm{~B}$ \\
\hline W4-115 & 0 & - & 332.855774 & 0.144966 & 0.120 & -21.93 & 10.51 & 0.294 & $\mathrm{~B}$ \\
\hline W4-117 & 0 & - & 333.500244 & 0.317248 & 0.163 & -22.88 & 11.18 & 0.548 & $\mathrm{R}$ \\
\hline W4-120 & 1 & - & 333.735870 & 0.478156 & 0.094 & -21.35 & 10.45 & 0.447 & $\mathrm{~B}$ \\
\hline W4-121 & 0 & - & 333.027924 & 0.586307 & 0.063 & -20.49 & 10.31 & 0.629 & $\mathrm{R}$ \\
\hline W4-122 & 0 & - & 333.153503 & 0.600898 & 0.096 & -21.42 & 10.64 & 0.591 & $\mathrm{R}$ \\
\hline W4-124 & 0 & - & 333.321320 & 0.838570 & 0.107 & -21.83 & 10.56 & 0.374 & B \\
\hline W4-125 & 0 & - & 334.522858 & -0.001675 & 0.102 & -21.89 & 10.69 & 0.464 & $\mathrm{~B}$ \\
\hline W4-126 & 0 & - & 334.505646 & -0.022670 & 0.118 & -22.02 & 10.66 & 0.395 & B \\
\hline W4-128 & 0 & - & 333.872803 & 0.116215 & 0.125 & -22.61 & 10.73 & 0.241 & $\mathrm{~B}$ \\
\hline W4-129 & 0 & - & 333.909607 & 0.135223 & 0.090 & -22.15 & 10.85 & 0.518 & $\mathrm{~B}$ \\
\hline W4-130 & 0 & - & 334.423370 & 0.152145 & 0.061 & -21.28 & 10.56 & 0.574 & $\mathrm{R}$ \\
\hline W4-131 & 3 & $\mathrm{~L}$ & 334.073944 & 0.165063 & 0.180 & -22.98 & 11.27 & 0.593 & $\mathrm{R}$ \\
\hline W4-132 & 0 & - & 334.453735 & 0.174981 & 0.107 & -21.67 & 10.72 & 0.572 & $\mathrm{R}$ \\
\hline W4-134 & 0 & - & 333.960541 & 0.214290 & 0.159 & -23.19 & 11.32 & 0.569 & $\mathrm{R}$ \\
\hline W4-135 & 0 & - & 334.013916 & 0.213259 & 0.120 & -22.87 & 11.24 & 0.611 & $\mathrm{R}$ \\
\hline W4-136 & 0 & - & 334.565125 & 0.268002 & 0.061 & -20.31 & 10.18 & 0.576 & $\mathrm{R}$ \\
\hline W4-137 & 0 & - & 334.393616 & 0.304381 & 0.116 & -22.07 & 10.64 & 0.361 & $\mathrm{~B}$ \\
\hline W4-138 & 0 & - & 334.344666 & 0.352445 & 0.132 & -22.39 & 10.96 & 0.527 & $\mathrm{~B}$ \\
\hline W4-139 & 0 & - & 334.697968 & 0.398587 & 0.066 & -20.42 & 9.94 & 0.325 & $\mathrm{~B}$ \\
\hline W4-141 & 0 & - & 334.083710 & 0.520163 & 0.105 & -22.13 & 10.69 & 0.384 & $\mathrm{~B}$ \\
\hline W4-142 & 0 & - & 334.232239 & 0.671036 & 0.087 & -21.97 & 10.60 & 0.362 & $\mathrm{~B}$ \\
\hline W4-145 & 0 & - & 334.394898 & 0.811042 & 0.165 & -23.01 & 11.19 & 0.517 & B \\
\hline W4-146 & 0 & - & 335.154083 & 0.011333 & 0.126 & -22.29 & 11.01 & 0.610 & $\mathrm{R}$ \\
\hline W4-149 & 0 & - & 335.221314 & 0.046047 & 0.150 & -22.43 & 11.02 & 0.573 & $\mathrm{R}$ \\
\hline W4-151 & 0 & - & 335.692719 & 0.161847 & 0.080 & -20.97 & 10.34 & 0.482 & $\mathrm{~B}$ \\
\hline W4-152 & 0 & - & 335.376465 & 0.271377 & 0.142 & -22.24 & 10.91 & 0.539 & $\mathrm{R}$ \\
\hline W4-153 & 3 & $\mathrm{~L}$ & 335.127350 & 0.296558 & 0.064 & -21.14 & 10.59 & 0.649 & $\mathrm{R}$ \\
\hline W4-154 & 0 & - & 335.040833 & 0.327715 & 0.167 & -23.14 & 11.32 & 0.583 & $\mathrm{R}$ \\
\hline W4-155 & 0 & - & 335.182129 & 0.340280 & 0.073 & -21.20 & 10.40 & 0.458 & $\mathrm{~B}$ \\
\hline
\end{tabular}


TABLE 4-Continued

\begin{tabular}{|c|c|c|c|c|c|c|c|c|c|}
\hline ID & Confidence $^{\mathrm{b}}$ & Features $^{\mathrm{c}}$ & $\begin{array}{c}\text { RA } \\
{[\mathrm{J} 2000]}\end{array}$ & $\begin{array}{c}\text { Dec } \\
{[\mathrm{J} 2000]}\end{array}$ & $z_{p h o t}$ & $\begin{array}{c}\mathrm{M}_{r}^{\prime \mathrm{d}} \\
{[\mathrm{mag}]}\end{array}$ & $\begin{array}{c}\mathrm{M}_{\star}^{\mathrm{e}} \\
{\left[\log \left(\mathrm{M}_{\odot}\right)\right]}\end{array}$ & $\begin{array}{c}\left(g^{\prime}-r^{\prime}\right)^{\mathrm{f}} \\
{[\mathrm{mag}]}\end{array}$ & Red/Blue ${ }^{g}$ \\
\hline W4-157 & 2 & - & 335.343994 & 0.461429 & 0.077 & -21.05 & 10.47 & 0.570 & $\mathrm{R}$ \\
\hline W4-158 & 0 & - & 335.340393 & 0.467835 & 0.120 & -22.13 & 10.93 & 0.594 & $\mathrm{R}$ \\
\hline W4-159 & 4 & $\mathrm{~S}$ & 334.748718 & 0.662590 & 0.193 & -24.06 & 11.59 & 0.491 & $\mathrm{~B}$ \\
\hline W4-160 & 0 & - & 334.843048 & 0.750440 & 0.121 & -22.51 & 11.08 & 0.593 & $\mathrm{R}$ \\
\hline W4-161 & 0 & - & 335.592804 & 0.751369 & 0.093 & -21.59 & 10.73 & 0.613 & $\mathrm{R}$ \\
\hline W4-162 & 0 & - & 335.400574 & 0.847896 & 0.193 & -23.60 & 11.36 & 0.459 & B \\
\hline W4-163 & 0 & - & 330.500336 & 0.909949 & 0.159 & -22.81 & 11.27 & 0.653 & $\mathrm{R}$ \\
\hline W4-164 & 0 & - & 330.662903 & 0.924946 & 0.091 & -21.19 & 10.28 & 0.346 & $\mathrm{~B}$ \\
\hline W4-165 & 2 & - & 330.103088 & 0.980357 & 0.144 & -22.28 & 10.93 & 0.540 & $\mathrm{R}$ \\
\hline W4-167 & 0 & - & 330.801117 & 1.016565 & 0.154 & -22.88 & 11.25 & 0.617 & $\mathrm{R}$ \\
\hline W4-169 & 0 & - & 330.626007 & 1.052597 & 0.077 & -21.84 & 10.73 & 0.521 & B \\
\hline W4-173 & 3 & $\mathrm{~S}, \mathrm{~L}$ & 330.549530 & 1.103121 & 0.153 & -22.69 & 11.14 & 0.584 & $\mathrm{R}$ \\
\hline W4-174 & 0 & - & 330.227508 & 1.199446 & 0.150 & -23.30 & 11.43 & 0.619 & $\mathrm{R}$ \\
\hline W4-175 & 0 & - & 330.741913 & 1.276678 & 0.104 & -21.97 & 10.88 & 0.611 & $\mathrm{R}$ \\
\hline W4-176 & 0 & - & 330.244995 & 1.429781 & 0.168 & -22.74 & 11.18 & 0.600 & $\mathrm{R}$ \\
\hline W4-177 & 4 & $\mathrm{~S}$ & 330.747742 & 1.491192 & 0.101 & -21.98 & 10.85 & 0.574 & $\mathrm{R}$ \\
\hline W4-178 & 0 & - & 330.557068 & 1.484962 & 0.180 & -23.00 & 11.28 & 0.600 & $\mathrm{R}$ \\
\hline W4-179 & 0 & - & 330.375824 & 1.506501 & 0.081 & -21.30 & 10.53 & 0.534 & $\mathrm{R}$ \\
\hline W4-182 & 0 & - & 330.707916 & 1.637985 & 0.197 & -23.15 & 11.19 & 0.467 & $\mathrm{~B}$ \\
\hline W4-184 & 0 & - & 331.239654 & 0.845040 & 0.130 & -22.40 & 11.00 & 0.560 & $\mathrm{R}$ \\
\hline W4-185 & 4 & $\mathrm{~L}, \mathrm{H}, \mathrm{F}$ & 331.455017 & 1.034040 & 0.162 & -23.08 & 11.37 & 0.650 & $\mathrm{R}$ \\
\hline W4-186 & 0 & - & 331.658508 & 1.021953 & 0.074 & -20.77 & 10.31 & 0.527 & $\mathrm{R}$ \\
\hline W4-188 & 0 & - & 331.541107 & 1.084808 & 0.180 & -23.69 & 11.59 & 0.626 & $\mathrm{R}$ \\
\hline W4-189 & 4 & M & 331.601654 & 1.112513 & 0.145 & -22.57 & 11.06 & 0.558 & $\mathrm{R}$ \\
\hline W4-190 & 0 & - & 331.116974 & 1.242247 & 0.175 & -23.49 & 11.37 & 0.498 & B \\
\hline W4-191 & 0 & - & 331.176971 & 1.351165 & 0.151 & -23.19 & 11.41 & 0.647 & $\mathrm{R}$ \\
\hline W4-192 & 0 & - & 331.746124 & 1.362379 & 0.192 & -23.33 & 11.43 & 0.614 & $\mathrm{R}$ \\
\hline W4-194 & 3 & $\mathrm{~L}$ & 331.392883 & 1.545326 & 0.167 & -23.83 & 11.59 & 0.576 & $\mathrm{R}$ \\
\hline W4-196 & 1 & - & 331.638397 & 1.613127 & 0.147 & -23.10 & 11.30 & 0.579 & $\mathrm{R}$ \\
\hline W4-197 & 0 & - & 331.476685 & 1.657902 & 0.108 & -22.35 & 10.97 & 0.552 & $\mathrm{R}$ \\
\hline W4-198 & 0 & - & 332.392670 & 1.057576 & 0.162 & -22.82 & 10.83 & 0.264 & B \\
\hline W4-199 & 0 & - & 332.280945 & 1.076237 & 0.102 & -21.41 & 10.52 & 0.487 & B \\
\hline W4-200 & 0 & - & 332.155182 & 1.149590 & 0.145 & -22.83 & 11.23 & 0.615 & $\mathrm{R}$ \\
\hline W4-201 & 4 & $\mathrm{~S}, \mathrm{~L}$ & 332.338562 & 1.223990 & 0.124 & -22.12 & 10.96 & 0.622 & $\mathrm{R}$ \\
\hline W4-202 & 2 & - & 332.365387 & 1.283633 & 0.117 & -22.73 & 11.17 & 0.595 & $\mathrm{R}$ \\
\hline W4-203 & 2 & - & 332.356567 & 1.308342 & 0.135 & -23.01 & 11.27 & 0.584 & $\mathrm{R}$ \\
\hline W4-205 & 0 & - & 332.573914 & 1.329177 & 0.132 & -22.19 & 10.90 & 0.546 & $\mathrm{R}$ \\
\hline W4-206 & 0 & - & 332.368805 & 1.400959 & 0.114 & -22.02 & 10.89 & 0.596 & $\mathrm{R}$ \\
\hline W4-207 & 0 & - & 332.156891 & 1.390052 & 0.065 & -20.83 & 10.08 & 0.306 & $\mathrm{~B}$ \\
\hline W4-208 & 0 & - & 332.761810 & 1.437543 & 0.180 & -23.51 & 11.52 & 0.634 & $\mathrm{R}$ \\
\hline W4-209 & 0 & - & 332.632843 & 1.454486 & 0.180 & -22.98 & 11.31 & 0.628 & $\mathrm{R}$ \\
\hline W4-211 & 0 & - & 332.248901 & 1.469085 & 0.174 & -22.76 & 11.15 & 0.565 & $\mathrm{R}$ \\
\hline W4-212 & 0 & - & 332.391937 & 1.481176 & 0.180 & -23.43 & 11.45 & 0.595 & $\mathrm{R}$ \\
\hline W4-213 & 0 & - & 332.431061 & 1.538875 & 0.057 & -21.24 & 10.54 & 0.566 & $\mathrm{R}$ \\
\hline
\end{tabular}


TABLE 4-Continued

\begin{tabular}{|c|c|c|c|c|c|c|c|c|c|}
\hline ID & Confidence $^{\mathrm{b}}$ & Features $^{c}$ & $\begin{array}{c}\mathrm{RA} \\
{[\mathrm{J} 2000]}\end{array}$ & $\begin{array}{c}\text { Dec } \\
{[\mathrm{J} 2000]}\end{array}$ & $z_{p h o t}$ & $\begin{array}{c}\mathrm{M}_{r}^{\prime \mathrm{d}} \\
{[\mathrm{mag}]}\end{array}$ & $\begin{array}{c}\mathrm{M}_{\star}{ }^{\mathrm{e}} \\
{\left[\log \left(\mathrm{M}_{\odot}\right)\right]}\end{array}$ & $\begin{array}{c}\left(g^{\prime}-r^{\prime}\right)^{\mathrm{f}} \\
{[\mathrm{mag}]}\end{array}$ & Red/Blue ${ }^{g}$ \\
\hline W4-214 & 0 & - & 332.638886 & 1.525781 & 0.111 & -22.01 & 10.67 & 0.403 & $\mathrm{~B}$ \\
\hline W4-215 & 0 & - & 332.419403 & 1.518399 & 0.072 & -20.78 & 10.04 & 0.289 & B \\
\hline W4-217 & 4 & M & 332.558563 & 1.589804 & 0.094 & -21.25 & 10.52 & 0.546 & $\mathrm{R}$ \\
\hline W4-218 & 4 & M & 332.557922 & 1.588671 & 0.077 & -20.84 & 10.45 & 0.625 & $\mathrm{R}$ \\
\hline W4-219 & 0 & - & 332.552154 & 1.601283 & 0.088 & -21.16 & 10.41 & 0.477 & $\mathrm{~B}$ \\
\hline W4-220 & 4 & $\mathrm{M}, \mathrm{A}$ & 332.208527 & 1.654040 & 0.151 & -22.80 & 11.24 & 0.628 & $\mathrm{R}$ \\
\hline W4-222 & 0 & - & 332.778076 & 1.672177 & 0.076 & -20.86 & 10.29 & 0.475 & $\mathrm{~B}$ \\
\hline W4-224 & 1 & - & 332.304504 & 1.731201 & 0.116 & -21.74 & 10.60 & 0.440 & $\mathrm{~B}$ \\
\hline W4-225 & 4 & $\mathrm{~F}$ & 333.104736 & 0.853247 & 0.167 & -23.29 & 11.38 & 0.585 & $\mathrm{R}$ \\
\hline W4-226 & 0 & - & 333.227387 & 0.914140 & 0.062 & -21.24 & 10.28 & 0.333 & $\mathrm{~B}$ \\
\hline W4-227 & 1 & - & 333.206543 & 1.008695 & 0.082 & -21.38 & 10.58 & 0.556 & $\mathrm{R}$ \\
\hline W4-228 & 1 & - & 332.981781 & 1.068207 & 0.090 & -21.23 & 10.47 & 0.507 & $\mathrm{~B}$ \\
\hline W4-229 & 0 & - & 332.999237 & 1.201210 & 0.118 & -21.71 & 10.41 & 0.287 & $\mathrm{~B}$ \\
\hline W4-230 & 0 & - & 332.965088 & 1.281956 & 0.108 & -22.00 & 10.88 & 0.595 & $\mathrm{R}$ \\
\hline W4-231 & 2 & - & 333.366913 & 1.306768 & 0.142 & -22.20 & 10.62 & 0.294 & $\mathrm{~B}$ \\
\hline W4-234 & 0 & - & 332.966949 & 1.576547 & 0.157 & -22.66 & 11.12 & 0.573 & $\mathrm{R}$ \\
\hline W4-236 & 0 & - & 334.537140 & 0.994265 & 0.113 & -22.20 & 10.90 & 0.544 & $\mathrm{R}$ \\
\hline W4-237 & 0 & - & 334.152771 & 1.005529 & 0.150 & -22.44 & 11.12 & 0.657 & $\mathrm{R}$ \\
\hline W4-238 & 1 & - & 333.880676 & 1.029724 & 0.058 & -21.02 & 10.52 & 0.628 & $\mathrm{R}$ \\
\hline W4-239 & 0 & - & 334.670441 & 1.056372 & 0.152 & -22.77 & 11.10 & 0.516 & $\mathrm{~B}$ \\
\hline W4-240 & 0 & - & 334.118988 & 1.058140 & 0.137 & -22.15 & 10.84 & 0.512 & $\mathrm{~B}$ \\
\hline W4-242 & 0 & - & 334.458588 & 1.098715 & 0.088 & -21.70 & 10.55 & 0.406 & $\mathrm{~B}$ \\
\hline W4-243 & 0 & - & 334.679169 & 1.171040 & 0.082 & -21.25 & 10.48 & 0.504 & $\mathrm{~B}$ \\
\hline W4-245 & 4 & $\mathrm{~A}$ & 333.995300 & 1.365095 & 0.075 & -21.56 & 10.66 & 0.557 & $\mathrm{R}$ \\
\hline W4-247 & 4 & $\mathrm{~F}$ & 333.912994 & 1.463670 & 0.120 & -22.35 & 11.06 & 0.636 & $\mathrm{R}$ \\
\hline W4-248 & 0 & - & 334.213287 & 1.462910 & 0.060 & -20.78 & 9.90 & 0.162 & $\mathrm{~B}$ \\
\hline W4-249 & 2 & - & 334.212463 & 1.463410 & 0.093 & -21.62 & 10.34 & 0.248 & $\mathrm{~B}$ \\
\hline W4-250 & 0 & - & 334.648499 & 1.496185 & 0.093 & -21.67 & 10.69 & 0.545 & $\mathrm{R}$ \\
\hline W4-252 & 0 & - & 334.550354 & 1.501998 & 0.069 & -20.77 & 10.07 & 0.315 & $\mathrm{~B}$ \\
\hline W4-254 & 0 & - & 334.690674 & 1.537719 & 0.138 & -23.24 & 11.20 & 0.444 & $\mathrm{~B}$ \\
\hline W4-255 & 0 & - & 334.693420 & 1.531309 & 0.126 & -21.93 & 10.60 & 0.369 & $\mathrm{~B}$ \\
\hline W4-257 & 3 & $\mathrm{~F}, \mathrm{~A}$ & 334.561340 & 1.631843 & 0.154 & -23.30 & 11.43 & 0.628 & $\mathrm{R}$ \\
\hline W4-260 & 0 & - & 334.742859 & 1.743470 & 0.192 & -23.82 & 11.64 & 0.627 & $\mathrm{R}$ \\
\hline W4-261 & 0 & - & 333.983093 & 1.742156 & 0.078 & -21.29 & 10.35 & 0.376 & $\mathrm{~B}$ \\
\hline W4-263 & 0 & - & 334.894196 & 0.984520 & 0.084 & -21.32 & 10.41 & 0.420 & $\mathrm{~B}$ \\
\hline W4-265 & 0 & - & 334.930267 & 1.052131 & 0.071 & -20.69 & 10.27 & 0.520 & $\mathrm{R}$ \\
\hline W4-266 & 0 & - & 335.659058 & 1.115576 & 0.060 & -20.60 & 10.40 & 0.670 & $\mathrm{R}$ \\
\hline W4-267 & 4 & M & 335.167786 & 1.230395 & 0.153 & -23.53 & 11.41 & 0.528 & $\mathrm{~B}$ \\
\hline W4-269 & 2 & - & 335.194702 & 1.258995 & 0.182 & -23.00 & 11.28 & 0.597 & $\mathrm{R}$ \\
\hline W4-270 & 2 & - & 335.196197 & 1.260140 & 0.180 & -23.00 & 11.28 & 0.599 & $\mathrm{R}$ \\
\hline W4-271 & 0 & - & 335.701508 & 1.275821 & 0.168 & -22.72 & 11.11 & 0.550 & $\mathrm{R}$ \\
\hline W4-272 & 0 & - & 335.220062 & 1.287796 & 0.121 & -22.36 & 10.96 & 0.537 & $\mathrm{R}$ \\
\hline W4-273 & 0 & - & 335.699402 & 1.295124 & 0.104 & -21.59 & 10.41 & 0.325 & $\mathrm{~B}$ \\
\hline W4-275 & 0 & - & 335.083710 & 1.394875 & 0.170 & -23.46 & 11.40 & 0.541 & $\mathrm{~B}$ \\
\hline
\end{tabular}


TABLE 4-Continued

\begin{tabular}{|c|c|c|c|c|c|c|c|c|c|}
\hline ID & Confidence $^{\mathrm{b}}$ & Features $^{\mathrm{c}}$ & $\begin{array}{c}\text { RA } \\
{[\mathrm{J} 2000]}\end{array}$ & $\begin{array}{c}\text { Dec } \\
{[\mathrm{J} 2000]}\end{array}$ & $z_{p h o t}$ & $\begin{array}{c}\mathrm{M}_{r}^{\prime \mathrm{d}} \\
{[\mathrm{mag}]}\end{array}$ & $\begin{array}{c}\mathrm{M}_{\star}^{\mathrm{e}} \\
{\left[\log \left(\mathrm{M}_{\odot}\right)\right]}\end{array}$ & $\begin{array}{c}\left(g^{\prime}-r^{\prime}\right)^{\mathrm{f}} \\
{[\mathrm{mag}]}\end{array}$ & Red/Blue ${ }^{g}$ \\
\hline W4-276 & 0 & - & 335.216370 & 1.430157 & 0.091 & -21.46 & 10.71 & 0.639 & $\mathrm{R}$ \\
\hline W4-277 & 0 & - & 334.875824 & 1.515994 & 0.082 & -21.71 & 10.59 & 0.441 & B \\
\hline W4-278 & 4 & M & 335.647675 & 1.527969 & 0.141 & -22.29 & 10.96 & 0.566 & $\mathrm{R}$ \\
\hline W4-279 & 3 & $\mathrm{~F}$ & 335.305054 & 1.576374 & 0.131 & -22.27 & 11.00 & 0.609 & $\mathrm{R}$ \\
\hline W4-280 & 1 & - & 335.171448 & 1.604952 & 0.081 & -21.69 & 10.58 & 0.444 & B \\
\hline W4-282 & 0 & - & 334.761597 & 1.685503 & 0.179 & -23.05 & 11.26 & 0.558 & $\mathrm{R}$ \\
\hline W4-283 & 0 & - & 334.827576 & 1.714247 & 0.168 & -22.70 & 11.13 & 0.575 & $\mathrm{R}$ \\
\hline W4-284 & 0 & - & 334.852508 & 1.746216 & 0.120 & -22.56 & 11.15 & 0.638 & $\mathrm{R}$ \\
\hline W4-285 & 0 & - & 335.271515 & 1.777235 & 0.146 & -22.68 & 11.00 & 0.465 & B \\
\hline W4-287 & 0 & - & 330.313019 & 1.847237 & 0.048 & -20.37 & 10.14 & 0.520 & $\mathrm{R}$ \\
\hline W4-288 & 0 & - & 330.649506 & 1.871501 & 0.076 & -21.54 & 10.57 & 0.484 & B \\
\hline W4-289 & 0 & - & 330.124329 & 1.870875 & 0.117 & -21.92 & 10.86 & 0.605 & $\mathrm{R}$ \\
\hline W4-290 & 0 & - & 330.343780 & 1.928869 & 0.158 & -22.76 & 11.18 & 0.591 & $\mathrm{R}$ \\
\hline W4-291 & 2 & - & 330.706085 & 1.940658 & 0.124 & -22.43 & 11.06 & 0.605 & $\mathrm{R}$ \\
\hline W4-293 & 0 & - & 330.138550 & 2.036495 & 0.109 & -21.98 & 10.64 & 0.392 & B \\
\hline W4-294 & 0 & - & 330.143433 & 2.041096 & 0.072 & -21.14 & 10.19 & 0.284 & $\mathrm{~B}$ \\
\hline W4-295 & 0 & - & 330.226624 & 2.044110 & 0.130 & -22.28 & 10.95 & 0.554 & $\mathrm{R}$ \\
\hline W4-297 & 0 & - & 330.616455 & 2.130686 & 0.118 & -22.03 & 10.73 & 0.451 & B \\
\hline W4-298 & 0 & - & 330.279175 & 2.219401 & 0.090 & -21.49 & 10.65 & 0.572 & $\mathrm{R}$ \\
\hline W4-299 & 0 & - & 330.233368 & 2.219552 & 0.164 & -23.20 & 11.35 & 0.590 & $\mathrm{R}$ \\
\hline W4-301 & 0 & - & 330.647919 & 2.407765 & 0.076 & -20.87 & 10.25 & 0.444 & B \\
\hline W4-303 & 4 & $\mathrm{~S}, \mathrm{~A}$ & 330.872162 & 2.531194 & 0.180 & -22.90 & 11.28 & 0.637 & $\mathrm{R}$ \\
\hline W4-305 & 0 & - & 330.673248 & 2.611628 & 0.180 & -23.19 & 11.32 & 0.567 & $\mathrm{R}$ \\
\hline W4-309 & 1 & - & 331.565430 & 1.795392 & 0.166 & -23.51 & 11.40 & 0.527 & B \\
\hline W4-310 & 0 & - & 331.478516 & 1.816255 & 0.150 & -22.48 & 11.05 & 0.574 & $\mathrm{R}$ \\
\hline W4-311 & 3 & $\mathrm{~F}$ & 331.190857 & 1.835212 & 0.085 & -21.40 & 10.42 & 0.401 & B \\
\hline W4-312 & 4 & $\mathrm{~L}$ & 331.204681 & 1.845583 & 0.074 & -20.92 & 10.03 & 0.226 & B \\
\hline W4-313 & 3 & $\mathrm{~S}$ & 331.312103 & 1.941748 & 0.127 & -22.53 & 11.00 & 0.518 & $\mathrm{~B}$ \\
\hline W4-314 & 1 & - & 331.186676 & 1.948029 & 0.065 & -20.59 & 9.83 & 0.168 & B \\
\hline W4-316 & 1 & - & 331.731049 & 2.034368 & 0.150 & -23.19 & 11.36 & 0.606 & $\mathrm{R}$ \\
\hline W4-317 & 4 & $\mathrm{~L}, \mathrm{H}, \mathrm{M}$ & 331.672119 & 2.045805 & 0.085 & -21.38 & 10.58 & 0.555 & $\mathrm{R}$ \\
\hline W4-318 & 0 & - & 331.682770 & 2.054486 & 0.062 & -20.31 & 9.81 & 0.242 & B \\
\hline W4-319 & 0 & - & 331.533752 & 2.101310 & 0.139 & -22.24 & 10.96 & 0.581 & $\mathrm{R}$ \\
\hline W4-320 & 0 & - & 331.338165 & 2.145282 & 0.071 & -20.94 & 10.32 & 0.478 & B \\
\hline W4-321 & 0 & - & 331.770477 & 2.145975 & 0.086 & -21.18 & 10.33 & 0.402 & $\mathrm{~B}$ \\
\hline W4-322 & 1 & - & 331.444397 & 2.146207 & 0.091 & -21.36 & 10.57 & 0.550 & $\mathrm{R}$ \\
\hline W4-323 & 4 & $\mathrm{~S}, \mathrm{~F}$ & 331.405029 & 2.193817 & 0.156 & -23.29 & 11.36 & 0.565 & $\mathrm{R}$ \\
\hline W4-325 & 0 & - & 331.277008 & 2.301760 & 0.067 & -20.78 & 10.02 & 0.262 & B \\
\hline W4-326 & 0 & - & 331.436462 & 2.280555 & 0.081 & -21.02 & 10.06 & 0.214 & $\mathrm{~B}$ \\
\hline W4-328 & 0 & - & 331.690216 & 2.297623 & 0.149 & -22.99 & 11.14 & 0.475 & B \\
\hline W4-332 & 1 & - & 331.583038 & 2.429217 & 0.134 & -22.64 & 10.83 & 0.322 & B \\
\hline W4-333 & 3 & $\mathrm{~S}$ & 331.000885 & 2.471788 & 0.073 & -21.05 & 10.32 & 0.436 & B \\
\hline W4-335 & 0 & - & 331.253784 & 2.505939 & 0.150 & -23.53 & 11.38 & 0.501 & B \\
\hline W4-340 & 0 & - & 332.725098 & 1.850783 & 0.063 & -20.29 & 9.85 & 0.286 & $\mathrm{~B}$ \\
\hline
\end{tabular}


TABLE 4-Continued

\begin{tabular}{|c|c|c|c|c|c|c|c|c|c|}
\hline ID & Confidence $^{\mathrm{b}}$ & Features $^{\mathrm{c}}$ & $\begin{array}{c}\mathrm{RA} \\
{[\mathrm{J} 2000]}\end{array}$ & $\begin{array}{c}\text { Dec } \\
{[\mathrm{J} 2000]}\end{array}$ & $z_{\text {phot }}$ & $\begin{array}{c}\mathrm{M}_{r}^{\prime \mathrm{d}} \\
{[\mathrm{mag}]}\end{array}$ & $\begin{array}{c}\mathrm{M}_{\star}{ }^{\mathrm{e}} \\
{\left[\log \left(\mathrm{M}_{\odot}\right)\right]}\end{array}$ & $\begin{array}{c}\left(g^{\prime}-r^{\prime}\right)^{\mathrm{f}} \\
{[\mathrm{mag}]}\end{array}$ & Red/Blue ${ }^{g}$ \\
\hline W4-341 & 1 & - & 332.268005 & 1.901855 & 0.112 & -22.07 & 10.88 & 0.571 & $\mathrm{R}$ \\
\hline W4-342 & 0 & - & 332.448578 & 1.924362 & 0.076 & -21.09 & 10.22 & 0.333 & $\mathrm{~B}$ \\
\hline W4-343 & 3 & $\mathrm{~A}$ & 332.776520 & 2.092740 & 0.095 & -21.27 & 10.23 & 0.276 & $\mathrm{~B}$ \\
\hline W4-350 & 4 & $\mathrm{~S}$ & 333.318695 & 2.351277 & 0.092 & -21.89 & 10.84 & 0.598 & $\mathrm{R}$ \\
\hline W4-354 & 0 & - & 332.854309 & 2.566752 & 0.180 & -22.90 & 11.26 & 0.611 & $\mathrm{R}$ \\
\hline W4-358 & 0 & - & 334.257874 & 1.830785 & 0.170 & -23.24 & 11.38 & 0.603 & $\mathrm{R}$ \\
\hline W4-359 & 1 & - & 334.105347 & 1.929412 & 0.170 & -22.89 & 11.14 & 0.510 & $\mathrm{~B}$ \\
\hline W4-361 & 0 & - & 334.175262 & 2.026385 & 0.185 & -23.33 & 11.24 & 0.449 & $\mathrm{~B}$ \\
\hline W4-362 & 0 & - & 334.698029 & 2.089893 & 0.170 & -22.79 & 10.65 & 0.113 & $\mathrm{~B}$ \\
\hline W4-363 & 0 & - & 333.927856 & 2.105751 & 0.137 & -22.37 & 11.07 & 0.636 & $\mathrm{R}$ \\
\hline W4-364 & 1 & - & 333.868530 & 2.133758 & 0.148 & -22.62 & 11.03 & 0.510 & $\mathrm{~B}$ \\
\hline W4-365 & 3 & M & 333.851929 & 2.145244 & 0.166 & -22.71 & 11.22 & 0.646 & $\mathrm{R}$ \\
\hline W4-367 & 0 & - & 334.354736 & 2.275227 & 0.106 & -21.73 & 10.60 & 0.446 & $\mathrm{~B}$ \\
\hline W4-368 & 0 & - & 334.374145 & 2.408052 & 0.124 & -22.72 & 10.99 & 0.440 & B \\
\hline W4-369 & 0 & - & 334.531738 & 2.422202 & 0.150 & -22.48 & 11.11 & 0.632 & $\mathrm{R}$ \\
\hline W4-370 & 0 & - & 334.579498 & 2.485579 & 0.069 & -20.98 & 10.28 & 0.423 & $\mathrm{~B}$ \\
\hline W4-371 & 0 & - & 334.276520 & 2.508918 & 0.120 & -22.21 & 11.01 & 0.640 & $\mathrm{R}$ \\
\hline W4-372 & 0 & - & 333.857056 & 2.537548 & 0.123 & -22.06 & 10.64 & 0.360 & $\mathrm{~B}$ \\
\hline W4-374 & 1 & - & 333.966217 & 2.538991 & 0.159 & -22.71 & 11.21 & 0.639 & $\mathrm{R}$ \\
\hline W4-375 & 0 & - & 333.895935 & 2.558254 & 0.194 & -23.29 & 11.29 & 0.506 & $\mathrm{~B}$ \\
\hline W4-376 & 0 & - & 334.031006 & 2.596407 & 0.123 & -22.85 & 11.05 & 0.444 & $\mathrm{~B}$ \\
\hline W4-378 & 0 & - & 334.001007 & 2.653258 & 0.150 & -22.39 & 10.85 & 0.434 & $\mathrm{~B}$ \\
\hline W4-380 & 0 & - & 330.015655 & 2.783270 & 0.180 & -23.36 & 11.43 & 0.599 & $\mathrm{R}$ \\
\hline W4-383 & 0 & - & 330.892151 & 3.038593 & 0.137 & -22.22 & 10.95 & 0.583 & $\mathrm{R}$ \\
\hline W4-385 & 4 & $\mathrm{~S}$ & 330.643189 & 3.274862 & 0.130 & -22.85 & 11.17 & 0.557 & $\mathrm{R}$ \\
\hline W4-386 & 0 & - & 330.869873 & 3.275786 & 0.109 & -22.09 & 10.75 & 0.449 & $\mathrm{~B}$ \\
\hline W4-387 & 0 & - & 330.722809 & 3.256110 & 0.150 & -22.59 & 11.14 & 0.615 & $\mathrm{R}$ \\
\hline W4-388 & 0 & - & 330.423370 & 3.273135 & 0.151 & -22.88 & 11.22 & 0.592 & $\mathrm{R}$ \\
\hline W4-395 & 0 & - & 330.507080 & 3.624160 & 0.179 & -23.14 & 11.29 & 0.559 & $\mathrm{R}$ \\
\hline W4-396 & 0 & - & 330.098846 & 3.643949 & 0.180 & -23.39 & 11.43 & 0.591 & $\mathrm{R}$ \\
\hline W4-398 & 0 & - & 331.063110 & 2.760770 & 0.063 & -20.28 & 9.90 & 0.337 & $\mathrm{~B}$ \\
\hline W4-401 & 0 & - & 331.101685 & 2.944743 & 0.121 & -22.26 & 11.02 & 0.634 & $\mathrm{R}$ \\
\hline W4-404 & 0 & - & 331.050537 & 3.230286 & 0.115 & -22.15 & 10.93 & 0.592 & $\mathrm{R}$ \\
\hline W4-407 & 2 & - & 331.197052 & 3.434962 & 0.180 & -23.48 & 11.47 & 0.598 & $\mathrm{R}$ \\
\hline W4-408 & 0 & - & 330.947479 & 3.505455 & 0.178 & -23.39 & 11.43 & 0.591 & $\mathrm{R}$ \\
\hline W4-409 & 0 & - & 331.445862 & 3.611482 & 0.096 & -21.63 & 10.61 & 0.492 & $\mathrm{~B}$ \\
\hline W4-413 & 0 & - & 331.943329 & 3.241427 & 0.125 & -22.20 & 10.78 & 0.437 & B \\
\hline W4-414 & 2 & - & 331.919373 & 3.258077 & 0.156 & -23.05 & 11.21 & 0.517 & $\mathrm{~B}$ \\
\hline W4-416 & 4 & $\mathrm{~L}, \mathrm{~F}$ & 331.918701 & 3.296956 & 0.164 & -22.61 & 11.10 & 0.571 & $\mathrm{R}$ \\
\hline W4-417 & 0 & - & 332.618347 & 3.455970 & 0.103 & -22.01 & 10.54 & 0.293 & $\mathrm{~B}$ \\
\hline W4-418 & 0 & - & 332.602661 & 3.446751 & 0.089 & -21.57 & 10.36 & 0.286 & $\mathrm{~B}$ \\
\hline W4-422 & 0 & - & 330.779755 & 3.776597 & 0.101 & -21.53 & 10.65 & 0.557 & $\mathrm{R}$ \\
\hline W4-423 & 0 & - & 330.397613 & 3.791380 & 0.077 & -21.02 & 10.44 & 0.552 & $\mathrm{R}$ \\
\hline W4-424 & 0 & - & 330.077820 & 4.167262 & 0.165 & -22.53 & 10.82 & 0.354 & $\mathrm{~B}$ \\
\hline
\end{tabular}


TABLE 4-Continued

\begin{tabular}{|c|c|c|c|c|c|c|c|c|c|}
\hline ID & Confidence $^{\mathrm{b}}$ & Features $^{\mathrm{c}}$ & $\begin{array}{c}\text { RA } \\
{[\mathrm{J} 2000]}\end{array}$ & $\begin{array}{c}\text { Dec } \\
{[\mathrm{J} 2000]}\end{array}$ & $z_{p h o t}$ & $\begin{array}{c}\mathrm{M}_{r}^{\prime \mathrm{d}} \\
{[\mathrm{mag}]}\end{array}$ & $\begin{array}{c}\mathrm{M}_{\star}{ }^{\mathrm{e}} \\
{\left[\log \left(\mathrm{M}_{\odot}\right)\right]}\end{array}$ & $\begin{array}{c}\left(g^{\prime}-r^{\prime}\right)^{\mathrm{f}} \\
{[\mathrm{mag}]}\end{array}$ & Red/Blue \\
\hline W4-426 & 0 & - & 330.108063 & 4.203234 & 0.147 & -22.55 & 10.95 & 0.464 & B \\
\hline W4-427 & 2 & - & 330.515106 & 4.298555 & 0.163 & -23.46 & 11.48 & 0.615 & $\mathrm{R}$ \\
\hline W4-429 & 4 & $\mathrm{~F}$ & 330.042114 & 4.361153 & 0.160 & -23.19 & 11.37 & 0.612 & $\mathrm{R}$ \\
\hline W4-431 & 3 & M & 331.306305 & 3.723338 & 0.159 & -22.98 & 11.35 & 0.671 & $\mathrm{R}$ \\
\hline W4-432 & 0 & - & 331.361664 & 3.916549 & 0.150 & -22.47 & 11.08 & 0.606 & $\mathrm{R}$ \\
\hline W4-433 & 0 & - & 331.056335 & 4.024874 & 0.150 & -22.94 & 11.29 & 0.631 & $\mathrm{R}$ \\
\hline W4-436 & 0 & - & 331.090271 & 4.118608 & 0.144 & -22.44 & 11.02 & 0.567 & $\mathrm{R}$ \\
\hline W4-437 & 0 & - & 330.992920 & 4.119300 & 0.148 & -22.36 & 10.90 & 0.491 & B \\
\hline W4-438 & 0 & - & 331.239075 & 4.187174 & 0.150 & -23.17 & 11.37 & 0.619 & $\mathrm{R}$ \\
\hline W4-440 & 0 & - & 331.135590 & 4.200491 & 0.095 & -21.93 & 10.78 & 0.533 & $\mathrm{R}$ \\
\hline W4-441 & 0 & - & 331.194428 & 4.439025 & 0.119 & -22.16 & 10.87 & 0.533 & $\mathrm{R}$ \\
\hline W4-442 & 0 & - & 331.088165 & 4.506465 & 0.127 & -21.94 & 10.59 & 0.362 & B \\
\hline W4-444 & 0 & - & 332.082214 & 3.675448 & 0.196 & -23.27 & 11.33 & 0.546 & $\mathrm{R}$ \\
\hline W4-448 & 1 & - & 332.644531 & 3.891357 & 0.151 & -22.82 & 11.23 & 0.614 & $\mathrm{R}$ \\
\hline W4-449 & 0 & - & 332.092743 & 3.993267 & 0.129 & -22.35 & 10.94 & 0.526 & B \\
\hline W4-452 & 0 & - & 332.673401 & 4.136741 & 0.180 & -23.03 & 11.31 & 0.611 & $\mathrm{R}$ \\
\hline W4-453 & 0 & - & 332.111176 & 4.225845 & 0.080 & -21.08 & 10.23 & 0.345 & B \\
\hline W4-456 & 3 & S & 332.441864 & 4.386792 & 0.170 & -23.77 & 11.60 & 0.603 & $\mathrm{R}$ \\
\hline W4-457 & 0 & - & 332.115387 & 4.392965 & 0.075 & -20.72 & 10.13 & 0.387 & B \\
\hline W4-458 & 4 & $\mathrm{~L}, \mathrm{~F}$ & 332.648132 & 4.445655 & 0.120 & -22.04 & 10.89 & 0.594 & $\mathrm{R}$ \\
\hline
\end{tabular}

${ }^{\mathrm{a}}$ The full version of this table appears in the electronic version of this paper.

${ }^{\mathrm{b}}$ Confidence class. $4=$ certain, $3=$ probable, $2=$ possible, $1=$ hint, $0=$ none. See text for details.

${ }^{\mathrm{c}}$ Feature classifications. 'L'=linear, 'A'=arm, 'H'=shell, 'S'=stream, 'F'=fan, 'M'=miscellaneous/diffuse.

${ }^{\mathrm{d}}$ Rest-frame r'-band magnitude

${ }^{\mathrm{e}}$ Base-10 logarithm of stellar mass in solar units.

${ }^{\mathrm{f}}$ Rest-frame color.

${ }^{\mathrm{g}}$ Flag indicating blue cloud (B) or red sequence (R). 